\title{
SOLUTION OF THE BASIN PROBLEM FOR HÉNON-LIKE ATTRACTORS
}

\author{
Michael BENEDICKS and Marcelo VIANA
}
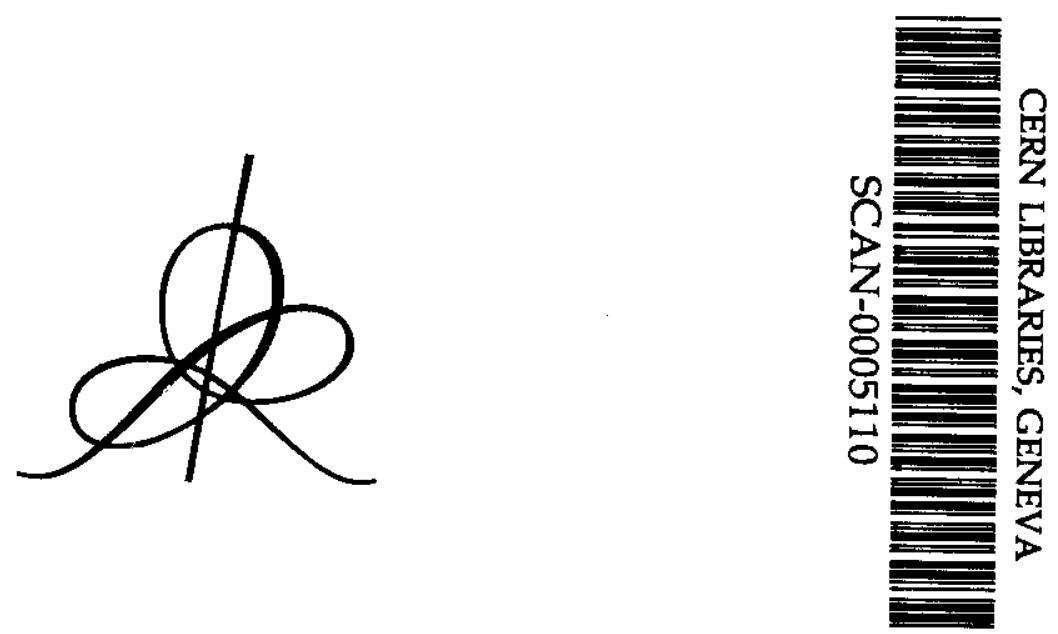

Institut des Hautes Études Scientifiques

35 , route de Chartres

91440 - Bures-sur-Yvette (France)

Juillet 1999

IHES/M/99/60 


\title{
Solution of the basin problem for Hénon-like attractors
}

\author{
Michael Benedicks and Marcelo Viana *
}

July 1999

\begin{abstract}
For a large class of non-uniformly hyperbolic attractors of dissipative diffeomorphisms, we prove that there are no "holes" in the basin of attraction: stable manifolds of points in the attractor fill-in a full Lebesgue measure subset. Then, Lebesgue almost every point in the basin is generic for the SRB (Sinai-Ruelle-Bowen) measure of the attractor. This solves a problem posed by Sinai and by Ruelle, for this class of systems.
\end{abstract}

\section{Introduction}

For most dynamical systems, are time averages well-defined at Lebesgue almost every orbit ? This is always the case if the system preserves Lebesgue measure, according to the ergodic theorem. However, in general this fundamental problem, raised by Sinai and by Ruelle in the seventies, remains essentially open. For dissipative systems one usually looks at the dynamics in the basin of each attractor, and then the problem can be restated Is almost every orbit in the basin of attraction asymptotic to some orbit contained in the attractor? Is it generic for some SRB measure supported in the attractor? See [11, Sec. IV], [16, p. 148].

By attractor one means a compact invariant subset $\Lambda$ of the phase space $M$, dynamically indecomposable (e.g. $\Lambda$ contains dense orbits), and whose basin $B(\Lambda)=\{z \in M$ whose future orbit accumulates on $\Lambda\}$ is a large set (a neighbourhood of $\Lambda$, say). One wants to focus on attractors having some degree of robustness under perturbations of the dynamical system, which is often associated to some form of hyperbolicity. Let $W^{s}(\xi)$ denote the set of all $z \in M$ whose orbit approaches the orbit of a point $\xi$ as time goes to $+\infty$. Then the first question may be formulated

(B1) does $B(\Lambda)=\bigcup_{\xi \in \Lambda} W^{s}(\xi)$ up to a zero Lebesgue measure set ?

Suppose $\Lambda$ supports an invariant ergodic measure $\mu$ which is hyperbolic (all the Lyapunov exponents are nonzero) and whose conditional measures on unstable manifolds are absolutely continuous with respect to Lebesgue measure. Then, see [17], [18], $\mu$ is an $S R B$ measure, in

\footnotetext{
"This work was partially supported by a STINT grant. M.B. is partially supported by the NFR and the
} Göran Gustafsson Foundation. M.V. is partially supported by Pronex - Dynamical Systems and Faperj. 
the sense that its basin $B(\mu)$ has positive Lebesgue measure in $M$. By definition, $B(\mu)$ is the set of points $z \in M$ such that the time average of every continuous function $\varphi: M \rightarrow \mathbb{R}$ cn the orbit of $z$ exists and coincides with the space average $\int \varphi d \mu$ (one also says that $z$ is a generic point for $\mu$ ). Then, supposing that $\mu$ is unique,

(B2) does $B(\mu)=B(\Lambda)$ up to a zero Lebesgue measure set ?

It is now classical that both versions (B1) and (B2) of the basin problem have an affirmative answer in the case of uniformly hyperbolic (Axiom A) attractors, where a main ingredient is the uniform shadowing property. See [21], [20], [19], [8], [7]. On the other hand, although these problems have been around for some time, little is known in the non-uniformly hyperbolic setting: exceptions include the geometric Lorenz-like attractors [1], [12] (for which a stable foliation exists, essentially, by definition), and systems preserving a smooth ergodic measure $\mu$ (where $B(\mu)$ has full measure as a direct consequence of the ergodic theorem).

Here we give a positive solution to the basin problem for Hénon-like attractors. This type of attractor was first constructed in [2], where it was shown that the Hénon model

$$
h(x, y)=\left(1-a x^{2}+y, b x\right)
$$

has a "strange" (non-hyperbolic) attractor for a set of values of the parameters $(a, b)$ with positive Lebesgue measure. Based on these methods, attractors combining hyperbolic behaviour with presence of "folding" regions were shown to occur persistently in certain general bifurcation mechanisms [15], [10]. Moreover, it was proved in [5] that all these Hénon-like attractors support a unique invariant measure $\mu$ as above. It is for this class of systems that we state our results.

Theorem A. Let $\Lambda$ be a Hénon-like attractor of a surface diffeomorphism $f: M \rightarrow M$. Then, through Lebesgue almost every point $z \in B(\Lambda)$ passes a stable leaf $W^{s}(\xi)$ of some $\xi \in \Lambda$ : $\operatorname{dist}\left(f^{n}(z), f^{n}(\xi)\right) \rightarrow 0$ exponentially fast as $n \rightarrow+\infty$. Theorem B. Denoting by $\mu$ the SRB measure of $f$ on $\Lambda$, then for Lebesgue almost every
$z \in B(\Lambda)$ one has

$$
\lim _{n \rightarrow+\infty} \frac{1}{n} \sum_{j=0}^{n-1} \varphi\left(f^{j}(z)\right)=\int \varphi d \mu \text { for every continuous } \varphi: M \rightarrow \mathbb{R} .
$$

As a by-product of the proofs we also get that the stable manifold $W^{s}(P)$ is dense in $B(\Lambda)$, where $P \in \Lambda$ denotes a hyperbolic saddle-point such that $\Lambda=\operatorname{closure}\left(W^{u}(P)\right)$.

Let us mention a few other recent developments in the ergodic theory of Hénon-like attractors. It is shown in [6] that the system $(f, \mu)$ has exponential decay of correlations in the space of Hölder continuous observable functions. In [4] we prove that these systems are stochastically stable with respect to small random perturbations with absolutely continuous transitions. Finally, an alternative approach to the construction of the SRB measure has been announced by
the authors of [13].

According to [22], persistent Hénon-like attractors exist for diffeomorphisms on manifolds of arbitrary dimension. Not all our arguments carry on to higher dimensions, but we expect Theorems A and B to hold in such generality, and it would be interesting to establish this.

We recall in the next section those known properties of Hénon-like attractors that are used in our arguments. In the remainder of this Introduction we comment on ideas involved in the 
proof of Theorem A. We begin by pointing out that a local version of the basin problem, which holds in the Axiom A case, is false for Hénon-like maps: the local stable sets of points $\xi \in \Lambda$, do not fill-in a full Lebesgue measure subset of a neighbourhood of the attractor. This means that global control of the stable lamination is needed in the present case.

Our strategy is to identify a positive Lebesgue measure set $H$ formed ty stable leaves of points in $\Lambda$, and to show that almost every $z \in B(\Lambda)$ eventually reaches this set. The points in $H$ are characterised by a bounded recurrence property that ensures that their orbits do not return too often to the folding region and, most important, these returns are always "tangential" in the sense of [2], see Section 2 below.

The arrival time to $H$, a parameter of "nonlocalness", depends in a ve:y discontinuous way on the point $z$. The fact that it is finite Lebesgue almost everywhere relies on a statistical argument that we present in Section 4. On its turn, this is based on a geometric pseudo-Markov construction on the basin of the attractor $\Lambda$, which we describe in Section 3 . A main feature is the following bounded geometry property: the set of points sharing a same finite itinerary is always a rectangle (bounded by a pair of stable segments and a pair of unstable segments).

In Section 5 we put these ideas together to prove Theorem A. Theorem B is a corollary, using the fact that $B(\mu)$ consists of entire stable leaves $W^{s}(\xi)$.

Acknowledgements: Part of this work was carried out at the University of Porto and the Schrödinger Institute in Vienna, besides our home institutions. We are also grateful to David Ruelle for inviting us to the IHÉS, where we finished the paper.

\section{Hénon-like attractors revisited}

Let us recall a number of known facts about Hénon-like attractors, from [2], [5], [15], that we use in Sections 3 through 5. First of all we fix some notations.

We deal with parametrised families of diffeomorphisms of the plane

$$
f(x, y)=f_{a}(x, y)=\left(1-a x^{2}, 0\right)+R(a, x, y),
$$

$R$ close to zero in the $C^{3}$ norm, which we call Hénon-like families. More precisely, we suppose that $\|R\|_{C^{3}} \leq J \sqrt{b}$ on $[1,2] \times[-2,2]^{2}$, with

$$
J^{-1} b \leq|\operatorname{det} D f| \leq J b \text { and }\|D(\log |\operatorname{det} D f|)\| \leq J,
$$

where $J>0$ is arbitrary and $b>0$ is taken sufficiently small. The quadratic family $1-a x^{2}$ may be replaced by any family of maps in some fixed $C^{3}$ neighbourhood of it. The Hénon model (1) is affinely conjugate to the map $f(x, y)=\left(1-a x^{2}+\sqrt{b} y, \sqrt{b} x\right)$, and so does fall into this
framework if $b$ is small.

We consider parameter values $a \in\left[a_{1}, a_{2}\right]$ with $1 \gg \delta \gg 2-a_{1} \gg 2-a_{2} \gg b$. In particular, $f$ has a unique fixed saddle-point $P$ such that $\Lambda=\operatorname{closure}\left(W^{u}(P)\right)$ is compact, indeed $\Lambda$ is contained in $(-2,2)^{2}$. It is well-known that the basin $B(\Lambda)$ has nonempty interior, see [2] or $[16$, App. III]. In all the situations concerned here it even contains a neighbourhood of $\Lambda$, see [3], [23], and Section 5 below. 


\subsection{Existence and properties}

Besides $J$, let $\sqrt{e}<\sigma_{1}<\sigma_{2}<2$ be fixed at the very beginning. For the next theoren, one also fixes constants $1 \gg \beta \gg \alpha>0$, and supposes $b \ll \delta \ll \alpha$. Throughout, we use $C>1$ to represent various large constants depending only on $J, \sigma_{1}, \sigma_{2}, \alpha$, or $\beta$ (not on $\delta$ or $b$ ). Analogously, $c \in(0,1)$ is a generic notation for small constants depending only on $J, \sigma_{1}, \sigma_{2}$, $\alpha$, or $\beta$. Let $I(\delta)=\{(x, y):|x|<\delta\}$. For $z \in W^{u}(P)$, let $t(z)$ be any norm 1 vector tangent to $W^{u}(P)$ at $z$ (the particular choice is irrelevant). Given a non-zero vector $v=\left(v_{1}, v_{2}\right) \in \mathbb{R}^{2}$, slope $v$ will always be taken with absolute values, i.e. slope $v=\left|v_{2}\right| /\left|v_{1}\right|$.

Theorem 2.1. Given any Hénon-like family, there exists a positive Lebesgue measure set $E$ such that for every $a \in E$ the map $f$ has a countable critical set $\mathcal{C} \subset W^{u}(P) \cap I(\delta)$ whose elements $\zeta$ satisfy

1. $t(\zeta)$ is almost horizontal and $t(f(\zeta))$ is almost vertical, in the sense that slope $t(\zeta) \leq C \sqrt{b}$ and slope $t(f(\zeta)) \geq c / \sqrt{b}$

2. $t(f(\zeta))$ is exponentially contracted and $w_{0}=(1,0)$ is exponentially expanded under positive iterates: $\left\|D f^{n}(f(\zeta)) t(f(\zeta))\right\| \leq(C b)^{n}$ and $\left\|D f^{n}(f(\zeta)) w_{0}\right\| \geq \sigma_{1}^{n}$ for all $n \geq 1$

3. if $f^{n}(\zeta) \in I(\delta)$ then there is $\zeta_{n} \in \mathcal{C}$ so that $\operatorname{dist}\left(f^{n}(\zeta), \zeta_{n}\right) \geq e^{-\alpha n}$ and there is a $C^{2}$ curve $L=\{(x, y(x))\}$ with $\left|y^{\prime}(x)\right| \leq 1 / 10$ and $\left|y^{\prime \prime}(x)\right| \leq 1 / 10$, tangent to $t\left(\zeta_{n}\right)$ at $\zeta_{n}$ and also containing $f^{n}(\zeta)$.

In addition, there exists $\zeta \in \mathcal{C}$ such that $\left\{f^{n}(\zeta): n \geq 0\right\}$ is dense in $\Lambda$.

Theorem 2.1 was first proved for the Hénon model in [2]. Then the arguments were extended to the Hénon-like case in [15]. The property in part 3 plays a central role in the proof, as well as in our own arguments here, and we shall return to comment on it. From now on we always suppose $a \in E$. The remaining statements in this subsection are part of the proof of this theorem, but we also make independent use of them in Sections $3-5$.

Proposition 2.2. 1. There exists $\zeta_{0}=\left(x_{0}, y_{0}\right) \in \mathcal{C}$ with $\left|x_{0}\right| \leq C \sqrt{b}$, so that $\mathcal{C} \cap G_{0}=\left\{\zeta_{0}\right\}$, where $G_{0}$ denotes the segment connecting $f\left(\zeta_{0}\right)$ to $f^{2}\left(\zeta_{0}\right)$ in $W^{u}(P)$;

2. denoting $G_{g}=f^{g}\left(G_{0}\right) \backslash f^{g-1}\left(G_{0}\right)$, then $\mathcal{C} \cap G_{g}$ is finite for every $g \geq 1$, and in fact $\mathcal{C} \cap G_{1}$ consists of a single point $\zeta_{1}$

3. for every $\zeta \in \mathcal{C} \cap G_{g}$ and $g \geq 0$, the segment $\gamma=\gamma(\zeta)$ of radius $\delta c^{g}$ around $\zeta$ in $W^{u}(P)$ may be written $\gamma=\{(x, y(x))\}$ with $\left|y^{\prime}(x)\right| \leq C \sqrt{b}$ and $\left|y^{\prime \prime}(x)\right| \leq C \sqrt{b}$;

4. given any $\zeta \in \mathcal{C} \cap G_{g}$ with $g>0$, there exist $\tilde{g}<g$ and $\tilde{\zeta} \in \mathcal{C} \cap G_{\bar{g}}$ with $\operatorname{dist}(\zeta, \tilde{\zeta}) \leq b^{g / 10}$.

The lower bound on the length of the segments $\gamma(\zeta)$ is important, so that we give a special name $\rho$ to the constant $c$ in the context of part 3 of the proposition. Moreover, we write $K$ for the large constant $C$, and call a $C^{2}(b)$ curve any curve $\{(x, y(x))\}$ with $\left|y^{\prime}(x)\right| \leq K \sqrt{b}$ and $\left|y^{\prime \prime}(x)\right| \leq K \sqrt{b}$. Note that the expanding eigenvalue of $D f(P)$ is negative and so $G_{0}$ is a neighbourhood of $P$ and $\zeta_{0}$ in $W^{u}(P)$. It is easy to see that $G_{0}$ and $G_{1}$ contain $C^{2}(b)$ curves extending from $x=-9 / 10$ to $x=9 / 10$. For $g \geq 0$, points in $G_{g}$ are said to be of generation $g$. 
Since every orbit in $B(\Lambda)$ must eventually enter $[-2,2]^{2}$, we may always aissume to be dealing with orbits which never leave $[-2,2]^{2}$ in positive time, and we do so. Given $\lambda>0$, a point $z=(x, y)$ is called $\lambda$-expanding if

$$
\left\|D f^{j}(z) w_{0}\right\| \geq \lambda^{j} \quad \text { for all } j \geq 1 .
$$

An important case is $z \in f(\mathcal{C})$, with $\lambda=\sigma_{1}$, cf. Theorem 2.1.2. We say that $z$ is $\lambda$-expanding up to time $n$ if the inequality in (2) holds for $1 \leq j \leq n$. We define the contracting direction of order $n \geq 1$ at $z$ as the tangent direction $e^{(n)}(z)$ that is most contracted by $D f^{n}(z)$. The next proposition summarises a number of results from [2, Section 5$]$ and [15, Section 6$]$. In the statement $\lambda>0$ and $\tau>0$ are arbitrary constants, with $\tau$ sufficiently small (e.g. $\tau \leq 10^{-20}$ ), and one assumes that $b$ is much smaller than either of them.

Proposition 2.3. Let $z$ be $\lambda$-expanding up to time $n \geq 1$, and $\xi$ satisfy $\operatorname{dist}\left(f^{j}(\xi), f^{j}(z)\right) \leq \tau^{j}$ for every $0 \leq j \leq n-1$. Then, for any point $\eta$ in the $\tau^{n}$-neighbourhood of $\xi$ and for every $1 \leq l \leq k \leq n$,

1. $e^{(k)}(\eta)$ is uniquely defined and nearly vertical: slope $e^{(k)}(\eta) \geq c / \sqrt{b}$;

2. angle $\left(e^{(l)}(\eta), e^{(k)}(\eta)\right) \leq(C b)^{l}$ and $\left\|D f^{l}(\eta) e^{(k)}(\eta)\right\| \leq(C b)^{l}$;

3. $\left\|D e^{(k)}(\eta)\right\| \leq C \sqrt{b}$ and $\left\|D^{2} e^{(k)}(\eta)\right\| \leq C \sqrt{b}$;

4. $\left\|D\left(D f^{l} e^{(k)}\right)(\eta)\right\| \leq(C b)^{l}$;

5. $1 / 10 \leq\left\|D f^{n}(\xi) w_{0}\right\| /\left\|D f^{n}(z) w_{0}\right\| \leq 10$ and angle $\left(D f^{n}(\xi) w_{0}, D f^{n}(x) w_{0}\right) \leq(\sqrt{C \tau})^{n}$.

Parts 3 and 4 are also true for the derivatives of $e^{(k)}$ and $D f^{l} e^{(k)}$ with respect to the parameter $a$. Throughout, we write expanding to mean $\lambda$-expanding for some $\lambda \geq e^{-20}$ (cf.
Remark 3.2).

Proposition 2.4. If $z$ is an expanding point then its stable set $W^{s}(z)$ contains a segment $\Gamma=\Gamma(z)=\{(x(y), y):|y| \leq 1 / 10\}$ with $\left|x^{\prime}\right| \leq C \sqrt{b}$ and $\left|x^{\prime \prime}\right| \leq C \sqrt{b}$, such that $z \in \Gamma$ and

$$
\operatorname{dist}\left(f^{n}(\xi), f^{n}(\eta)\right) \leq(C b)^{n} \operatorname{dist}(\xi, \eta), \quad \text { for all } \xi, \eta \in \Gamma \text { and } n \geq 1 .
$$

Moreover, if $z_{1}, z_{2}$ are expanding points then

$$
\text { angle }\left(t_{\Gamma}\left(\xi_{1}\right), t_{\Gamma}\left(\xi_{2}\right)\right) \leq C \sqrt{b} \operatorname{dist}\left(\xi_{1}, \xi_{2}\right), \quad \text { for every } \xi_{1} \in \Gamma\left(z_{1}\right), \xi_{2} \in \Gamma\left(z_{2}\right) \text {, }
$$

where $t_{\Gamma}\left(\xi_{i}\right)$ denotes any norm 1 vector tangent to $\Gamma\left(z_{i}\right)$ at $\xi_{i}, i=1,2$.

We call a long stable leaf any curve $\Gamma$ as in this proposition, and a stable leaf any compact curve having some iterate contained in a long stable leaf. The first part of the proposition is proved in [2, Section 5.3], the arguments extending directly to Hénon-like maps [15, Section 7C]. We sketch the proof, to explain how the second part, not explicitly stated in those papers, can be deduced from the construction.

One takes $\Gamma(z)=\lim \Gamma^{n}(z)$, where $\Gamma^{n}(z)$ is the integral curve of the direction field $e^{(n)}$ (the temporary stable leaf of order $n$ ) through the point $z$. One can check directly that the first integral curve $\Gamma^{l}(z)$ is long, meaning that it extends from $y=-1 / 10$ to $y=+1 / 10$. To prove 
that the same is true for all the $\Gamma^{n}(z)$ one uses induction. Let $\tau$ be fixed, as in Proposition 2.3, and $b \ll \tau$. Part 2 of that proposition implies

$$
\operatorname{dist}\left(f^{j}(\xi), f^{j}(z)\right) \leq(C b)^{j} \operatorname{dist}(\xi, z) \text { for any } 1 \leq j \leq n-1 \text { and } \xi \in \Gamma^{n-1}(z) .
$$

As a consequence, $e^{(n)}(\eta)$ is well-defined in the $\tau^{n}$-neighbourhood of $\Gamma^{n-1}(z)$. Mcreover, angle $\left(e^{(n-1)}, e^{(n)}\right) \leq(C b)^{n-1}$ ensures that the integral curve $\Gamma^{n}(z)$ does not leave this $\tau^{n}$, neighbourhood inside the region $|y| \leq 1 / 10$. Thus $\Gamma^{n}(z)$ must be long. The previous angle estimate also implies that $\lim \Gamma^{n}(z)$ does exist, and this is how one gets the first claim in
Proposition 2.4.

Now, given $z_{1}, z_{2}, \xi_{1} \in \Gamma\left(z_{1}\right), \xi_{2} \in \Gamma\left(z_{2}\right)$ as in the proposition, let $n$ be fixed such that $\tau^{n+1} \leq \operatorname{dist}\left(\xi_{1}, \xi_{2}\right) \leq \tau^{n}$. By parts 2 and 3 of Proposition 2.3 , angle $\left(t_{\Gamma}\left(\xi_{i}\right), e^{(n)}\left(\xi_{i}\right)\right) \leq(C b)^{n}$ for $i=1,2$, and angle $\left(e^{(n)}\left(\xi_{1}\right), e^{(n)}\left(\xi_{1}\right)\right) \leq C \sqrt{b} \operatorname{dist}\left(\xi_{1}, \xi_{2}\right)$. Then

$$
\text { angle }\left(t_{\Gamma}\left(\xi_{1}\right), t_{\Gamma}\left(\xi_{2}\right)\right) \leq 2(C b)^{n}+C \sqrt{b} \operatorname{dist}\left(\xi_{1}, \xi_{2}\right) \leq 2 C \sqrt{b} \operatorname{dist}\left(\xi_{1}, \xi_{2}\right) .
$$

This gives the last statement in Proposition 2.4.

Proposition 2.5. Given any $k \geq 1$, any $z \in[-2,2]^{2}$ satisfying $f^{j}(z) \notin I(\delta)$ for $0 \leq j<k$, and any tangent vector $v$ with $\|v\|=1$ and slope $v \leq 1 / 5$, then

$$
\operatorname{slope}\left(D f^{j}(z) v\right) \leq(C / \delta) \sqrt{b}<1 / 10 \text { and }\left\|D f^{j}(z) v\right\| \geq c \delta \sigma_{2}^{j}
$$

for $1 \leq j \leq k$. If either $z \in f(I(2 \delta))$ or $f^{k}(z) \in I(2 \delta)$ then $\left\|D f^{k}(z) v\right\| \geq \sigma_{2}^{k}$, and in the latter case we also have slope $\left(D f^{k}(z) v\right) \leq C \sqrt{b}$.

This means, in particular, that pieces of orbits outside $I(\delta)$ are (essentially) expanding. Similar statements are well-known for one-dimensional maps such as $x \mapsto 1-a x^{2}$. The proposition follows using a perturbation argument, see $[2$, Lemmas $4.5,4.6]$

Another important notion is that of bound period $p(n, \zeta)$ associated to a return $n$ of a critical point $\zeta \in \mathcal{C}$. These are defined through the following inductive procedure. If $n \geq 1$ does not belong to $[\nu+1, \nu+p(\nu, \zeta)]$ for any return $1 \leq \nu<n$, then $n$ is a (free) return for $\zeta$ if and only if $f^{n}(\zeta) \in I(\delta)$. Moreover, the bound period $p=p(n, \zeta)$ is the largest integer such that

$$
\operatorname{dist}\left(f^{n+j}(\zeta), f^{j}\left(\zeta_{n}\right)\right) \leq e^{-\beta j} \text { for all } 1 \leq j \leq p
$$

where $\zeta_{n}$ is the binding point of $f^{n}(\zeta)$, given by Theorem 2.1.3. If, on the contrary, $n$ is in $[\nu+1, \nu+p(\nu, \zeta)]$ for some previous return $1 \leq \nu<n$ then, by definition, $n$ is a (bound) return for $\zeta$ if and only if $n-\nu$ is a return for the binding point $\zeta_{\nu}$, and we let $p(n, \zeta)=p\left(n-\nu, \zeta_{\nu}\right)$. Up to a slight (and otherwise irrelevant) modification of these definitions, see [2, Section 6.2] or $[15$, Section 8$]$, we may suppose that bound periods are nested: whenever $n \in[\nu+1, \nu+p(\nu, \zeta)]$ then $n+p(n, \zeta) \leq \nu+p(\nu, \zeta)$, that is to say, the bound period associated to $n$ ends before the one associated to $\nu$.

We write $d_{n}(\zeta)=\operatorname{dist}\left(f^{n}(\zeta), \zeta_{n}\right)$ and $w_{j}(\zeta)=D f^{j}(f(\zeta)) w_{0}$ for $j \geq 0$. Proposition 2.6. Let $n \geq 1$ be a free return of $\zeta \in \mathcal{C}$, and $p=p(n, \zeta)$ be the corresponding
bound period. Then 
1. $(1 / 5) \log \left(1 / d_{n}(\zeta)\right) \leq p \leq 5 \log \left(1 / d_{n}(\zeta)\right) ;$

2. $\left\|w_{n+p}(\zeta)\right\| \geq \sigma_{1}^{(p+1) / 3}\left\|w_{n-1}(\zeta)\right\| \quad$ and $\quad \operatorname{slope} w_{n+p}(\zeta) \leq(C / \delta) \sqrt{b}$;

3. $\left\|w_{n+p}(\zeta)\right\| d_{n}(\zeta) \geq c e^{-\beta(p+1)} \dagger \mid w_{n-1}(\zeta) \| ;$

4. $\left\|w_{j}\left(f^{n+1}(\zeta)\right)\right\| \geq \sigma_{1}^{j} \quad$ for $1 \leq j \leq p, \quad$ and $\quad$ slope $w_{p}\left(f^{n+1}(\zeta)\right) \leq(C / \delta) \sqrt{b}$.

A main ingredient here is the property in Theorem 2.1.3. Actually, for free returns $n$, a curve $L$ as in the theorem may be taken tangent not only to $t\left(\zeta_{n}\right)$ at $\zeta_{n}$ but also to $w_{n-1}(\zeta)$ at $f^{n}(\zeta)$, see [2, Section 7.3] and [15, Lemma 9.5]. We shall explain below, in a more general context, how this is used in the proof.

\subsection{Dynamics on the unstable manifold}

The next proposition, appearing in [5], permits to extend to generic orbits in $W^{u}(P)$ the control given by the previous statements for orbits of critical points. This is a key step in the construction of the SRB measure of $f$ on $\Lambda$ that appeared in that paper, cf. Theorem 2.9 below.

Proposition 2.7. Let $\tilde{z} \in W^{u}(P)$ be such that $f^{n}(\tilde{z}) \notin \mathcal{C}$ for every $n \geq 1$. Then, given any $n \geq 1$ such that $f^{n}(\tilde{z}) \in I(\delta)$, there exists $\zeta_{n} \in \mathcal{C}$ and some $C^{2}$ curve $L=\{(x, y(x))\}$ with $\left|y^{\prime}\right| \leq 1 / 10$ and $\left|y^{\prime \prime}\right| \leq 1 / 10$, tangent to $t\left(\zeta_{n}\right)$ at $\zeta_{n}$ and also containing $f^{n}(\bar{z})$.

Let us elaborate a bit on the content and consequences of this proposition. Given a point $z \in W^{u}(P)$, fix $k \gg 1$ so that $\tilde{z}=f^{-k}(z)$ belongs to a small neighbourhood of $P$ in $W^{u}(P)$. We can now define returns, binding points, and bound periods for $\tilde{z}$ in the same way as we did before for critical points. That is, corresponding to a free return $n$ of $z$ we choose as binding point a critical point $\zeta_{n}$ as in the proposition, and define the bound period $p=p(n, \tilde{z})$ of $f^{n}(\tilde{z})$ with respect to this $\zeta_{n}$, cf. (3). As in the case of critical points, we may take the bound periods nested and we do so; see also comments following the next proposition.

We say that $z=f^{k}(\tilde{z})$ is a free point if $k$ is outside every bound period $\left[\nu+1, \nu+p\left(\nu, z_{0}\right)\right]$ of $z$. This is an intrinsic property of the point $z$ : the choice of $k$ is irrelevant, as long as it is large enough. We call a segment $\gamma \subset W^{u}(P)$ free if all its points are free.

While proving Proposition 2.7, it is shown in [5] that if $n$ is a free return for $z$ and $\gamma \subset W^{u}(P)$ is a free segment containing $f^{n}(\tilde{z})$, then a same binding point may be assigned to all the points in $\gamma \cap I(\delta)$. More precisely, there is a critical point $\zeta_{\gamma}$ and a curve $L$ as in the statement, tangent to $t\left(\zeta_{\gamma}\right)$ at $\zeta_{\gamma}$ and containing the whole $\gamma$. In particular, $L$ is tangent to $t(w)$ at every $w \in \gamma$. In some cases $\zeta_{\gamma} \in \gamma=L$, but it is not always possible to take $L \subset W^{u}(P)$. Given any maximal free segment $\gamma$ intersecting $I(\delta)$, we always fix $L$ and $\zeta_{\gamma}$ as above, and set $d_{\mathcal{C}}(w)=\operatorname{dist}\left(w, \zeta_{\gamma}\right)$ for each $w \in L$. Moreover, we define the binding period $p(z)$ of every $w \in L$ with respect to this $\zeta_{\gamma}$, cf. (3).

The following definition, directly motivated by the previous considerations, is a slight extension of notions with similar denominations in [2], [15], [5], [6]. Given points $p, q$ and tangent vectors $u, v$, we say that $p$ is in tangential position relative to $(q, v)$ if there exists a curve $\{(x, y(x))\}$ with $\left|y^{\prime}\right| \leq 1 / 5$ and $\left|y^{\prime \prime}\right| \leq 1 / 5$, tangent to $v$ at $q$ and also containing $p$. And we say $u$ at $p$.
that $(p, u$ in tangential position relative to $(q, v)$ if such a curve $L$ may be chosen tangent to 
Proposition 2.8. Given any curve $L$ as before, for every $z \in L$,

1. $(1 / 5) \log \left(1 / d_{\mathcal{C}}(z)\right) \leq p(z) \leq 5 \log \left(1 / d_{\mathcal{C}}(z)\right)$;

2. $\left\|D f^{p(z)+1}(z) t(z)\right\| \geq \sigma_{1}^{(p(z)+1) / 3} \quad$ and $\quad \operatorname{slope}\left(D f^{p(z)+1}(z) t(z)\right)<(C / \delta) \sqrt{b}$;

3. $\left\|D f^{p(z)+1}(z) t(z)\right\| d_{\mathcal{C}}(z) \geq c e^{-\beta(p(z)+1)}$;

4. $\left\|D f^{j}(f(z)) w_{0}\right\| \geq \sigma_{1}^{j} \quad$ for $1 \leq j \leq p(z)$, and $\operatorname{slope}\left(D f^{p(z)}(f(z)) w_{0}\right)<(C / \delta) \sqrt{b}$.

Propositions 2.6, 2.8 have similar proofs, based on the tangential position property. We outline the main steps since some features of these arguments are relevant for what follows; see also $[2$, Section 7.4$]$ and $[15$, Section 10].

Let $\zeta_{\gamma}=\left(x_{\gamma}, y\left(x_{\gamma}\right)\right), z=(x, y(x))$, and $p=p(z)$. We use $z(s)=(s, y(s))$ to represent a generic point in $L$, and write $w_{j}(s)=D f^{j}(f(z(s))) w_{0}$. First, one proves a distortion estimate, see [2, Lemma 7.8$]$ and [15, Lemma 10.5]:

$$
w_{j}(s)=\lambda(s)\left(w_{j}\left(x_{\gamma}\right)+\epsilon_{j}(s)\right), \quad c \leq \lambda(s) \leq C \text { and }\left\|\epsilon_{j}(s)\right\| \ll\left\|w_{j}\left(x_{\gamma}\right)\right\|,
$$

for every $1 \leq j \leq p$ and $s \in\left[x_{\gamma}, x\right]$. The main ingredient is provided by Theorem 2.1.3 associated to every return $j$ of $\zeta_{\gamma}$ there exists a critical point $\zeta_{j}$ with the tangential position property and $\operatorname{dist}\left(f^{j}\left(\zeta_{\gamma}\right), \zeta_{j}\right) \geq e^{-\alpha j}$. The proof of (4) combines this information with the fact that, cf. (3),

$$
\operatorname{dist}\left(f^{j}(z(s)), f^{j}\left(\zeta_{\gamma}\right)\right) \leq C e^{-\beta j} \ll e^{-\alpha j} \leq \operatorname{dist}\left(f^{j}\left(\zeta_{\gamma}\right), \zeta_{j}\right)
$$

for every return $1 \leq j \leq p$. From (4),

$$
\left\|w_{j}(s)\right\| \approx\left\|w_{j}\left(x_{\gamma}\right)\right\| \geq \sigma_{1}^{j}
$$

for all $1 \leq j \leq p$ and $s \in\left[x_{\gamma}, x\right]$, recall Theorem 2.1.2. In view of Proposition 2.3.1, for every $s \in\left[x_{\gamma}, x\right]$ the contracting direction of order $p$ at $f(z(s))$ is well defined and almost vertical: $e(s)=e^{(p)}(f(z(s)))$ is represented by a vector $\{\epsilon(s), 1)$ with $|\epsilon(s)| \leq C \sqrt{b}$. Cf. Theorem 2.1.2, the tangent direction to $W^{s}\left(f\left(\zeta_{\gamma}\right)\right)$ coincides with the tangent direction to $W^{u}(P)$ at $f\left(\zeta_{\gamma}\right)$. By Proposition 2.3.2, the angle between $e\left(\zeta_{\gamma}\right)$ and this tangent direction is bounded by $(C b)^{p}$.
Besides,

$$
\left|e^{\prime}(s)\right| \leq C \sqrt{b} \text { and }\left\|D f^{j}(f(z(s))) e(s)\right\| \leq(C b)^{j} \text { for all } 1 \leq j \leq p .
$$

See also $[2$, Section 5$]$ and $[15$, Section 6$]$.

Now one splits the tangent direction to $f(L)=\left\{\left(1-a s^{2}, 0\right)+R(a, s, y(s))\right\}=\{(\xi(s), \eta(s))\}$ into contracting and horizontal (expanding) components

$$
\left(\xi^{\prime}(s), \eta^{\prime}(s)\right)=\alpha(s) e(s)+\beta(s) w_{0} .
$$

Of course, $\alpha(s)=\eta^{\prime}(s)$ and $\beta(s)=\xi^{\prime}(s)-\epsilon(s) \eta^{\prime}(s)$. The assumption that $L=\{(s, y(s))\}$ is nearly horizontal, $\left|y^{\prime}\right|,\left|y^{\prime \prime}\right| \leq 1 / 5$, implies $\xi^{\prime \prime}(s) \approx-2 a$ and $\left|\eta^{\prime}(s)\right|,\left|\eta^{\prime \prime}(s)\right| \leq C \sqrt{b}$. Consequently, $\beta^{\prime}(s) \approx-2 a$ and $|\alpha(s)|,\left|\alpha^{\prime}(s)\right| \leq C \sqrt{b}$, in particular,

$$
\beta(s)-\beta\left(x_{\gamma}\right) \approx-2 a\left(s-x_{\gamma}\right) \approx \pm 2 a d_{\mathcal{C}}(z(s)) .
$$


The bound on the angle at $f\left(\zeta_{\gamma}\right)$ stated above gives $\left|\beta\left(x_{\gamma}\right)\right| \leq(C b)^{p}$. Usin: these estimates, one writes

$$
\begin{aligned}
f^{j+1}(z)-f^{j+1}\left(\zeta_{\gamma}\right) & =\int_{x_{\gamma}}^{x}\left(\alpha(s) D f^{j}(f(z(s))) e(s)+\beta(s) w_{j}(s)\right) d s \\
& =w_{j}\left(x_{\gamma}\right) \int_{x_{\gamma}}^{x} \lambda(s)\left(\beta(s)-\beta\left(x_{\gamma}\right)\right) d s+\delta_{j}
\end{aligned}
$$

with $\left\|\delta_{j}\right\| \ll(C b)^{j}+\left\|w_{j}\left(x_{\gamma}\right) \int_{x_{\gamma}}^{x} \lambda(s)\left(\beta(s)-\beta\left(x_{\gamma}\right)\right) d s\right\|$. By (4) and (7),

$$
\int_{x_{\gamma}}^{x} \lambda(s)\left(\beta(s)-\beta\left(x_{\gamma}\right)\right) d s \approx\left(x-x_{\gamma}\right)^{2} \approx d_{\mathcal{C}}(z)^{2}
$$

Taking $j=p$ in (8),

$$
e^{-\beta p} \approx \operatorname{dist}\left(f^{p+1}(z), f^{p+1}\left(\zeta_{\gamma}\right)\right) \approx\left\|w_{p}\left(x_{\gamma}\right)\right\| d_{\mathcal{C}}(z)^{2} .
$$

Part 1 of the proposition follows combining this with $4^{p} \geq\left\|w_{p}\left(x_{\gamma}\right)\right\| \geq \sigma_{1}^{p}$. From the relation $D f^{p+1}(z) t(z)=\beta(x) w_{p}(x)+\alpha(x) D f^{p}(f(z)) e(x)$, using (7) and $(9)$,

$$
\begin{aligned}
\left\|D f^{p+1}(z) t(z)\right\| & \geq c d_{\mathcal{C}}(z)\left\|w_{p}(x)\right\|-(C b)^{p}|| w_{p}(x) \|-C \sqrt{b}(C b)^{p} \\
& \geq c\left(e^{-\beta p}\left\|w_{p}(x)\right\|\right)^{1 / 2}-(4 C b)^{p} \geq \sigma_{1}^{(p+1) / 3}
\end{aligned}
$$

which proves the first statement in part 2 . The second statement uses

$$
\operatorname{slope}\left(D f^{p+1}(z) t(z)\right) \approx \operatorname{slope} w_{p}(x) \approx \operatorname{slope} w_{p}\left(x_{\gamma}\right),
$$

recall $(8),(4)$, together with the fact that $p+1$ does not belong to any bound period of $\zeta_{\gamma}$ (because bound periods are nested). To get part 3 ,

$$
\left\|D f^{p+1}(z) t(z)\right\| d_{\mathcal{C}}(z) \geq c d_{\mathcal{C}}(z)^{2}\left\|w_{p}(x)\right\|-(4 C b)^{p} \geq c e^{-\beta p} .
$$

The first half of part 4 follows from (6), and the second half is analogous to the slope statement in part 2. This ends our sketch of the proof of Proposition 2.8.

For Proposition 2.6 some extra care is needed: arguments as above assume properties of $w_{j}\left(\zeta_{n}\right), 1 \leq j \leq p\left(f^{n}(\zeta)\right)$, from the statement of Theorem 2.1, while the proposition itself is part of the proof of the theorem. To go around this, one begins by proving that

$$
p\left(f^{n}(\zeta)\right) \leq 5 \log \left(1 / d_{n}(\zeta)\right) \leq 5 \alpha n<n
$$

which ensures that such properties are used only in an inductive way.

It follows immediately from the proof that parts 2.4 of Proposition 2.8 remain true if one replaces $t(z)$ by any norm 1 tangent vector $v$ such that $(z, v)$ is in tangential position relative to $\left(\zeta_{\gamma}, t\left(\zeta_{\gamma}\right)\right)$. We want to point out that these arguments also allow for some freedom in the very definition of bound period. For instance, let $z(s) \in L$ with $s \in\left[x_{\gamma}, x\right]$ and

$$
|x-s| \leq c\left|x-x_{\gamma}\right|
$$


Taking $c \in(0,1)$ small enough, then (8) gives (distinguish two cases, depending on whether $\left\|w_{j}\left(x_{\gamma}\right)\right\| d_{\mathcal{C}}(z)^{2}$ is larger or smaller than $\left.(C b)^{j}\right)$

$$
\operatorname{dist}\left(f^{j+1}(z(s)), f^{j+1}\left(\zeta_{\gamma}\right)\right) \approx \operatorname{dist}\left(f^{j+1}(z), f^{j+1}\left(\zeta_{\gamma}\right)\right)
$$

(here $\approx$ means equality up to a factor bounded by 2 ) except, possibly, if both distances are smaller than $(C b)^{j}$. In any event,

$$
\begin{array}{ll}
\operatorname{dist}\left(f^{j}(z(s)), f^{j}\left(\zeta_{\gamma}\right)\right) & \leq 2 e^{-\beta j} \quad \text { for } 1 \leq j \leq p(z) \quad \text { and } \\
\operatorname{dist}\left(f^{j}(z(s)), f^{j}\left(\zeta_{\gamma}\right)\right) \geq \frac{1}{2} e^{-\beta j} & \text { for } j=p(z)+1,
\end{array}
$$

compare (5) and (9). Then the same arguments as before apply, to prove that parts $2-4$ of Proposition 2.8 remain true with $z(s)$ in the place of $z$, and $p(z)$ unchanged. So, one may just as well take $p(z(s))=p(z)$ for any such $s$. Accordingly, we always presume that, given any $z$ as before there exists a segment $L(z)$ with $z \in L(z) \subset L$ such that

$$
\text { length }(L(z)) \geq c d_{\mathcal{C}}(z) \text { and } p(\cdot) \text { is constant on } L(z) .
$$

We fix $c<1 / 10$ in (12), and denote it by $c_{1}$ from now on. A similar formulation is used in [6].

We also quote the main result of $[5]$ :

Theorem 2.9. There exists a unique $f$-invariant measure $\mu$ supported in $\Lambda$, having nonzero Lyapunov exponents almost everywhere, and whose conditional measures along unstable manifolds are absolutely continuous with respect to Lebesgue measure on these manifolds. The support of $\mu$ coincides with $\Lambda$, and the system $(f, \mu)$ is ergodic (even Bernoulli).

Given any segment $\gamma \subset W^{u}(P)$, almost every point in $\gamma$ (with respect to the arc-length measure) is generic for $\mu$. This can be read out from the proof as follows, see [5, Section 3]. Almost every point $z$ in $\gamma$ has infinitely many escape times $n_{i}$ : there exists a sequence $\gamma_{i}$ of neighbourhoods of $z$ in $\gamma$ such that $f^{n_{i}}\left(\gamma_{i}\right)$ is a long $C^{2}(b)$ curve (length $\approx \delta$ ), and the maps $f^{n_{i}} \mid \gamma_{i}$ have bounded distortion with respect to arc-length. A positive fraction, uniformly bounded away from zero, of the points in each of these long segments $f^{n_{i}}\left(\gamma_{i}\right)$ are generic for $\mu$. So, almost every point $z \in \gamma$ is a density point for the set of generic points, and this implies
the claim.

\section{Symbolic dynamics in the basin of attraction}

Here we construct a special sequence of partitions $\mathcal{P}_{n}$ in the basin of attraction, whose atoms are all rectangles, that is, regions bounded by two segments of $W^{u}(P)$ and by two stable leaves. A first step is Proposition 3.3: for each critical value $f(\zeta) \in f(\mathcal{C})$ there exists a sequence
$\Gamma_{r}=\Gamma_{r}(\zeta)$ of long stable leaves accumulating $W^{s}(f(\zeta))$ exponentially fast. Then we introduce a notion of itinerary of a point $z$ in the basin of attraction. It involves choosing a sequence of critical points $\zeta_{j}$ close to each iterate $f^{n_{j}}(z)$ that is near $x=0$, and describing the position of $f^{n_{j}(z)}$ relative to $\zeta_{j}$ in terms of these long stable leaves. The atoms of $\mathcal{P}_{n}$ are the sets of points sharing a same itinerary up to time $n$. 


\subsection{Constructing long stable leaves}

In all that follows $0<c_{1}<1 / 10$ is the constant we fixed before in the context of (12).

Lemma 3.1. Let $\gamma \subset W^{u}(P)$ be a free segment intersecting $I(\delta)$ such that length $(\gamma) \geq 2 c_{1} d_{\mathcal{C}}(z)$ for $z \in \gamma$. Then there exists $z_{\gamma} \in \gamma$ such that

$$
d_{\mathcal{C}}\left(f^{n}\left(z_{\gamma}\right)\right) \geq e^{-2 \beta n} \quad \text { for every return } n \geq 1 \text { of } z_{\gamma} .
$$

Proof. Let $\xi_{0}$ be the midpoint of $\gamma$ and $\gamma_{0}=\gamma \cap L\left(\xi_{0}\right)$, where $L\left(\xi_{0}\right)$ is the segment introduced in (12). Then

$$
\operatorname{length}\left(\gamma_{0}\right) \geq c_{1} d_{\mathcal{C}}\left(\xi_{0}\right),
$$

and there is $p_{0} \geq 1$ such that $p(z)=p_{0}$ for every $z \in \gamma_{0}$. Let $n_{0}>p_{0}$ be minimum such that $f^{n_{0}}\left(\gamma_{0}\right)$ intersects $I(\delta)$. Note that $f^{n_{0}}\left(\gamma_{0}\right)$ is a free segment. Then, by (13) and Propositions 2.5 and $2.8 .2-3$

$$
\begin{aligned}
\operatorname{length}\left(f^{n_{0}}\left(\gamma_{0}\right)\right) & \geq \sigma_{2}^{n_{0}-p_{0}-1} \text { length }\left(f^{p_{0}+1}\left(\gamma_{0}\right)\right) \geq \operatorname{length}\left(f^{p_{0}+1}\left(\gamma_{0}\right)\right) \\
& \geq \inf _{z \in \gamma_{0}}\left\|D f^{p_{0}+1}(z) t(z)\right\| c_{1} d_{\mathcal{C}}(z) \geq c c_{1} e^{-\beta\left(p_{0}+1\right)} \\
& \geq c c_{1} e^{-\beta n_{0}} \geq 20 e^{-2 \beta n_{0}}
\end{aligned}
$$

In the last inequality we use the remark that $n_{0}>p_{0}$ can be supposed arbitrarily large by decreasing $\delta>0$. As a consequence, there exists a segment $\tilde{\gamma}_{1} \subset f^{n_{0}}\left(\gamma_{0}\right)$ with

$$
\operatorname{length}\left(\tilde{\gamma}_{1}\right) \geq \frac{1}{4} \operatorname{length}\left(f^{n_{0}}\left(\gamma_{0}\right)\right) \geq 5 e^{-2 \beta n_{0}} \quad \text { and } \quad d_{\mathcal{C}}(z) \geq e^{-2 \beta n_{0}} \text { for } z \in \tilde{\gamma}_{1} .
$$

Let $\gamma_{1}=\tilde{\gamma}_{1} \cap L\left(\xi_{1}\right)$, where $\xi_{1}$ is the midpoint of $\tilde{\gamma}_{1}$. Note that $L\left(\xi_{1}\right)$ is made to contain a subsegment of $\tilde{\gamma}_{1}$ from $\xi_{1}$ to either the critical point or some boundary point of $\tilde{\gamma}_{1}$. Now we consider two different cases.

If (13) holds for $\gamma_{1}$, that is, if length $\left(\gamma_{1}\right) \geq c_{1} d_{\mathcal{C}}\left(\xi_{1}\right)$, then we just repeat the previous construction with $\gamma_{1}, \xi_{1}$, in the place of $\gamma_{0}, \xi_{0}$. Letting $p_{1}=p \mid \gamma_{1}$ and $n_{1}>p_{1}$ be minimum such that $f^{n_{1}}\left(\gamma_{1}\right)$ intersects $I(\delta)$, we find a segment $\tilde{\gamma}_{2} \subset f^{n_{1}}\left(\gamma_{1}\right)$ with

$$
\text { length }\left(\tilde{\gamma}_{2}\right) \geq 5 e^{-2 \beta n_{1}} \quad \text { and } \quad d_{\mathcal{C}}(z) \geq e^{-2 \beta n_{1}} \geq e^{-2 \beta\left(n_{0}+n_{1}\right)} \text { for } z \in \tilde{\gamma}_{2} .
$$

Now suppose length $\left(\gamma_{1}\right)<c_{1} d_{\mathcal{C}}\left(\xi_{1}\right)$. As length $\left(L\left(\xi_{1}\right)\right) \geq c_{1} d_{\mathcal{C}}\left(\xi_{1}\right)$, we must have

$$
\text { length }\left(\gamma_{1}\right) \geq \frac{1}{2} \text { length }\left(\tilde{\gamma}_{1}\right) \geq e^{-2 \beta n_{0}}
$$

Now, Propositions 2.5 and 2.8 .2 give

$$
\begin{aligned}
\operatorname{length}\left(f^{n_{1}}\left(\gamma_{1}\right)\right) & \geq \sigma_{2}^{n_{1}-p_{1}-1} \text { length }\left(f^{p_{1}+1}\left(\gamma_{1}\right)\right) \\
& \geq \sigma_{1}^{n_{1} / 3} \text { length }\left(\gamma_{1}\right) \geq 20 \text { length }\left(\gamma_{1}\right) \geq 20 e^{-2 \beta n_{0}} .
\end{aligned}
$$

Thus, there exists a segment $\tilde{\gamma}_{2} \subset f^{n_{1}}\left(\gamma_{1}\right)$ such that

$$
\operatorname{length}\left(\tilde{\gamma}_{2}\right) \geq \frac{1}{4} \text { length }\left(f^{n_{1}}\left(\gamma_{1}\right)\right) \geq 5 \text { length }\left(\gamma_{1}\right) \geq 5 e^{-2 \beta n_{0}}
$$


and

$$
d_{\mathcal{C}}(z) \geq e^{-2 \beta n_{0}} \geq e^{-2 \beta\left(n_{0}+n_{1}\right)} \text { for } z \in \tilde{\gamma}_{2} .
$$

Next, we take $\xi_{2}$ to be the midpoint of $\tilde{\gamma}_{2}$, and write $\gamma_{2}=\tilde{\gamma}_{2} \cap L\left(\xi_{2}\right)$. Then we apply the preceding steps with $\gamma_{2}, \xi_{2}$, in the place of $\gamma_{1}, \xi_{1}$ : as before, we distinguish two cases according to whether (13) holds for $\gamma_{2}, \xi_{2}$ or not. Iterating this procedure we construct a sequence $n_{i}$, $i \geq 0$, of large integers, and a sequence $\gamma_{i}, i \geq 0$, of segments in the unstable manifold $W^{u}(P)$, such that

$$
f^{n_{i-1}}\left(\gamma_{i-1}\right) \supset \gamma_{i} \text { and } d_{\mathcal{C}}(z) \geq e^{-2 \beta\left(n_{0}+\cdots+n_{i-1}\right)}
$$

for $z \in \gamma_{i}$ and every $i \geq 1$. The first property ensures that $f^{-\left(n_{0}+\cdots+n_{i-1}\right)}\left(\gamma_{i}\right), i \geq 1$, is a decreasing sequence of compact subsets of $\gamma$. Take $z_{\gamma}$ a point in the intersection of all these
subsets.

The conclusion of the lemma for the returns of such a $z_{\gamma}$ occurring at times $n_{0}+\cdots+n_{i-1}$, $i \geq 1$, follows directly from the second part of (16). Any other return $n$ is necessarily bcund, i.e. $n=n_{0}+\cdots+n_{i-1}+j$ for some $j \leq p_{i}$ and $i \geq 1$, and in this case the conclusion of the lemma is immediate:

$$
d_{\mathcal{C}}\left(f^{n}\left(z_{\gamma}\right)\right) \geq d_{\mathcal{C}}\left(f^{j}\left(\tilde{\zeta}_{i}\right)\right)-e^{-\beta j} \geq e^{-\alpha j}-e^{-\beta j} \geq e^{-2 \beta n},
$$

where $\tilde{\zeta}_{i}$ represents the binding point of $f^{n_{0}+\cdots+n_{i-1}}\left(z_{\gamma}\right)$.

Remark 3.1. We shall use a slightly stronger version of this lemma, where

$$
\text { length }(\gamma) \geq 2 c_{1} \frac{d_{\mathcal{C}}(z)}{\left|\log d_{\mathcal{C}}(z)\right|^{2}} \quad \text { for all } z \in \gamma,
$$

and the conclusion is as before. It follows from just the same proof, together with the following observation. Though we get length $\left(\gamma_{0}\right) \geq c_{1} d_{\mathcal{C}}\left(\xi_{0}\right)\left|\log d_{\mathcal{C}}\left(\xi_{0}\right)\right|^{-2}$ instead of (13), Proposition 2.8.1 ensures that (14) is not affected:

$$
\text { length }\left(f^{n_{0}}\left(\gamma_{0}\right)\right) \geq c c_{1} \frac{e^{-\beta\left(p_{0}+1\right)}}{\left|\log d_{\mathcal{C}}\left(\xi_{0}\right)\right|^{2}} \geq c c_{1} \frac{e^{-\beta\left(p_{0}+1\right)}}{\left(5 p_{0}\right)^{2}} \geq 20 e^{-2 \beta n_{0}},
$$

if $p_{0} \leq n_{0}$ is large enough.

In the next lemma we do not assume the point $z$ to be in the unstable manifold $W^{u}(P)$. Also, $w_{0}$ may be replaced by any norm 1 vector.

Lemma 3.2. Let $z \in I(\delta)$ and $k \geq 1$ be such that for every $1 \leq n \leq k$ with $f^{n}(z) \in I(\delta)$ there exists $\zeta_{n} \in \mathcal{C}$ satisfying

1. $\left(f^{n}(z), D f^{n-1}(f(z)) w_{0}\right)$ is in tangential position relative to $\left(\zeta_{n}, t\left(\zeta_{n}\right)\right)$;

2. $\operatorname{dist}\left(f^{n}(z), \zeta_{n}\right) \geq e^{-2 \beta n}$.

Then $f(z)$ is expanding up to time $k$, in fact, $\left\|D f^{j}(f(z)) w_{0}\right\| \geq \sigma_{1}^{j / 5}$ for every $1 \leq j \leq k$. 
Proof. We define a pair of sequences $n_{i}, p_{i}, i \geq 1$, as follows. As a first step, we take $n_{1}$ to be the smallest integer $n \geq 1$ such that $f^{n}(z) \in I(\delta)$. Then, for each $i \geq z$, we let $p_{i} \geq 1$ be maximum such that

$$
\operatorname{dist}\left(f^{n_{i}+j}(z), f^{j}\left(\zeta_{n_{i}}\right)\right) \leq e^{-\beta j} \text { for all } 1 \leq j \leq p_{i} .
$$

Finally, for each $i \geq 1$, we define $n_{i+1}$ to be the smallest integer $n>n_{i}+p_{i}$ such that $f^{n}(z) \in I(\delta)$.

Since we suppose $f(z) \in f(I(\delta))$, Proposition 2.5 gives $\left\|D f^{j}(f(z)) w_{0}\right\| \geq \sigma_{2}^{j}$ for every $1 \leq j \leq n_{1}-1$, which implies the conclusion of the lemma for $j<n_{1}$. Now we proceed by induction, in the following way. Let $i \geq 1$ and suppose we have shown that

$$
\left\|D f^{n_{i}-1}(f(z)) w_{0}\right\| \geq \sigma_{1}^{\left(n_{i}-1\right) / 3} .
$$

By assumption 1, cf. comments we made after Proposition 2.8,

$$
\left\|D f^{n_{i}+p_{i}}(f(z)) w_{0}\right\| \geq \sigma_{1}^{\left(p_{i}+1\right) / 3}\left\|D f^{n_{i}-1}(f(z)) w_{0}\right\| \geq \sigma_{1}^{\left(n_{i}+p_{i}\right) / 3} .
$$

As $\|D f\| \leq 4$, we conclude that, given any $0 \leq s<p_{i}$,

$$
\left\|D f^{n_{i}+s}(f(z)) w_{0}\right\| \geq 4^{s-p_{i}}\left\|D f^{n_{i}+p_{i}}(z) w_{0}\right\| \geq 4^{s-p_{i}} \sigma_{1}^{\left(n_{i}+p_{i}\right) / 3} .
$$

Using assumption 2 and Proposition 2.8.1, and taking $\beta>0$ sufficiently small,

$$
p_{i}-s \leq 5 \log \frac{1}{d\left(f^{n_{i}}(z), \zeta_{n_{i}}\right)} \leq 10 \beta n_{i} \leq \frac{\log \sigma_{1}}{10 \log 4}\left(n_{i}+s\right) .
$$

As a consequence, for every $0 \leq s \leq p_{i}$,

$$
\left\|D f^{n_{i}+s}(f(z)) w_{0}\right\| \geq \sigma_{1}^{-\left(n_{i}+s\right) / 10} \sigma_{1}^{\left(n_{i}+p_{i}\right) / 3} \geq \sigma_{1}^{\left(n_{i}+s\right) / 5} .
$$

This proves the lemma for $n_{i} \leq j \leq n_{i}+p_{i}$.

Next, Proposition 2.8 .2 gives slope $\left(D f^{n_{i}+p_{i}}(f(z)) w_{0}\right) \leq(C / \delta) \sqrt{b}<1 / 10$, and so we may use Proposition 2.5 to conclude that

$$
\left\|D f^{n_{i}+p_{i}+s}(f(z)) w_{0}\right\| \geq c \delta \sigma_{2}^{s}\left\|D f^{n_{i}+p_{i}}(f(z)) w_{0}\right\| \geq c \delta \sigma_{2}^{s} \sigma_{1}^{\left(n_{i}+p_{i}\right) / 3}
$$

for $1 \leq s<n_{i+1}-n_{i}-p_{i}$. Now, assumption 2 implies $2 \delta \geq e^{-2 \beta n_{i}}$, and so

$$
c \delta \sigma_{2}^{s} \sigma_{1}^{\left(n_{i}+p_{i}\right) / 3} \geq \frac{c}{2} \sigma_{2}^{s} \sigma_{1}^{\left(n_{i}+p_{i}\right) / 3} e^{-2 \beta n_{i}} \geq \frac{c}{2} \sigma_{1}^{\left(n_{i}+p_{i}+s\right) / 4} \geq \sigma_{1}^{\left(n_{i}+p_{i}+s\right) / 5} .
$$

In the second inequality we suppose $\beta>0$ is small with respect to $\log \sigma_{1}$, in the third one we use the fact that $n_{i}+p_{i}+s$ is very large (since $\delta$ is small). We have obtained the conclusion of the lemma also for $n_{i}+p_{i}<j<n_{i+1}$. Finally, the last part of Proposition 2.5 gives

$$
\begin{aligned}
\left\|D f^{n_{i+1}-1}(f(z)) w_{0}\right\| & \geq \sigma_{2}^{n_{i+1}-n_{i}-p_{i}-1}\left\|D f^{n_{i}+p_{i}}(f(z)) w_{0}\right\| \\
& \geq \sigma_{2}^{n_{i+1}-n_{i}-p_{i}-1} \sigma_{1}^{\left(n_{i}+p_{i}\right) / 3} \geq \sigma_{1}^{\left(n_{i+1}-1\right) / 3}
\end{aligned}
$$

which restores the induction hypothesis (17). 
Remark 3.2. Keeping assumption 1 of the lemma and replacing assumption 2 by

2. $\operatorname{dist}\left(f^{n}(z), \zeta_{n}\right) \geq e^{-5 n}$,

one still gets that $f(z)$ is expanding, in a weaker sense: $\left\|D f^{j}(f(z)) w_{0}\right\| \geq \lambda^{j}$ for $1 \leq j \leq k$, with $\lambda \geq 10^{-20}$. This is proved in the same way as the lemma, just replacing (18) by

$$
p_{i}-s \leq 5 \log \frac{1}{d\left(f^{n_{i}}(z), \zeta_{n_{i}}\right)} \leq 25 n_{i} \leq \frac{20 \log 10}{\log 4}\left(n_{i}+s\right) .
$$

It is convenient to take $\Delta=\log (1 / \delta)$ to be a (large) integer, and we do so in what follows.

Proposition 3.3. Given any critical value $f(\zeta) \in f(\mathcal{C})$ there exists a sequence of long stable leaves $\Gamma_{r}=\Gamma_{r}(\zeta)=\left\{\left(x_{r}(y), y\right):|y| \leq 1 / 10\right\}, r \geq \Delta$, accumulating $W^{s}(f(\zeta))$ exponentially fast from the left:

$$
e^{-2 r} \leq x^{s}(y)-x_{r}(y) \leq 10 e^{-2 r} \quad \text { for every } r \geq \Delta \text { and }|y| \leq 1 / 10,
$$

where $\left\{\left(x^{s}(y), y\right):|y| \leq 1 / 10\right\}=\Gamma^{s}$ is the long stable leaf through $f(\zeta)$.

Proof. Let us consider first $\zeta=\zeta_{0}$, the generation zero critical point in Proposition 2.2.1. Let $\gamma=\gamma\left(\zeta_{0}\right)$ be a segment of $W^{u}(P)$ extending $\delta$ to each side of $\zeta_{0}$. By Proposition 2.2.3, this is a $C^{2}(b)$ curve. For each $r \geq \Delta$, the set

$$
\left\{z \in \gamma: \frac{9}{10} e^{-r} \leq d\left(z, \zeta_{0}\right) \leq e^{-r}\right\}
$$

has two connected components. We shall we use $\gamma_{r}$ to denote either of the two. Then

$$
\text { length }\left(\gamma_{r}\right) \geq \frac{1}{10} e^{-r} \geq 2 c_{1} e^{-r} \geq 2 c_{1} d_{\mathcal{C}}(z) \text { for every } z \in \gamma_{r},
$$

and so, by Lemma 3.1 , there exists $z_{r} \in \gamma_{r}$ such that

$$
d_{\mathcal{C}}\left(f^{n}\left(z_{r}\right)\right)=d\left(f^{n}\left(z_{r}\right), \zeta_{n}\right) \geq e^{-2 \beta n}
$$

for every free return $n \geq 1$ of $z_{r}$. Here $\zeta_{n} \in \mathcal{C}$ is the binding point of $f^{n}\left(\zeta_{r}\right)$, recall Proposition 2.7 , so that $\left(f^{n}\left(z_{r}\right), t\left(f^{n}\left(z_{r}\right)\right)\right)$ is in tangential position relative to $\left(\zeta_{n}, t\left(\zeta_{n}\right)\right)$. We also
need

Lemma 3.4. $\left(f^{n}\left(z_{r}\right), D f^{n-1}\left(f\left(z_{r}\right)\right) w_{0}\right)$ is in tangential position relative to $\left(\zeta_{n}, t\left(\zeta_{n}\right)\right)$, for every free return $n \geq 1$ of $z_{r}$.

Proof. Take $p_{0} \geq 1$ maximum such that $\operatorname{dist}\left(f^{j}\left(z_{\tau}\right), \zeta\right) \leq e^{-\beta j}$ for all $1 \leq j \leq p_{0}$, and let $n_{1}$ be the first free return of $z_{r}$, in the sense that $n_{1}$ is the smallest integer larger than $p_{0}$ so that $f^{n_{1}}\left(z_{r}\right) \in I(\delta)$. Propositions 2.5 and $2.8 .4 \mathrm{imply}$

$$
\left\|D f^{n_{1}-1}\left(\eta_{r}\right) w_{0}\right\| \geq \sigma_{2}^{n_{1}-p_{0}-1}\left\|D f^{p_{0}}\left(\eta_{r}\right) w_{0}\right\| \geq \sigma_{2}^{n_{1}-p_{0}-1} \sigma_{1}^{p_{0}} \geq 2 .
$$

On the other hand, Propositions 2.5 and 2.8 .2 give

$$
\left\|D f^{n_{1}}\left(z_{r}\right) t\left(z_{r}\right)\right\| \geq \sigma_{2}^{n_{1}-p_{0}} \mid D f^{p_{0}}\left(z_{r}\right) t\left(z_{r}\right) \| \geq \sigma_{2}^{n_{1}-p_{0}} \sigma_{1}^{\left(p_{0}+1\right) / 3} \geq \sigma_{1}^{n_{1} / 3} .
$$


So,

$$
\left\|D f^{n_{1}-1}\left(\eta_{r}\right) t\left(\eta_{r}\right)\right\| \geq \frac{1}{5}\left\|D f^{n_{1}}\left(z_{r}\right) t\left(z_{r}\right)\right\| \geq \frac{1}{5} \sigma_{1}^{n_{1} / 3} \geq 2 .
$$

Since $\left|\operatorname{det} D f^{n_{1}-1}\left(\eta_{r}\right)\right| \leq(C b)^{n_{1}-1} \ll 1$, we deduce from (21) and (22) that

$$
\text { angle }\left(D f^{n_{1}-1}\left(\eta_{r}\right) w_{0}, D f^{n_{1}-1}\left(\eta_{r}\right) t\left(\eta_{r}\right)\right) \leq(C b)^{n_{1}-1} \ll \operatorname{dist}\left(f^{n_{1}-1}\left(\eta_{r}\right), \zeta_{n_{r}}\right) .
$$

By Proposition 2.7, there is a $C^{2}$ curve $L=\{(x, y(x))\}$ with $\left|y^{\prime}\right|,\left|y^{\prime \prime}\right| \leq 1 / 10$, tangent to $t\left(\zeta_{n}\right)$ at $\zeta_{n}$ and tangent to $D f^{n_{1}-1}\left(\eta_{r}\right) t\left(\eta_{r}\right)$ at $f^{n_{1}-1}\left(\eta_{r}\right)$. In view of (23), we may easily modify $L$ to a $C^{2}$ curve $\tilde{L}=\left\{(x, \tilde{y}(x)\}\right.$ with $\left|\tilde{y}^{\prime}\right|,\left|\tilde{y}^{\prime \prime}\right| \leq 1 / 5$ tangent to $t\left(\zeta_{n}\right)$ at $\zeta_{n}$ and to $D f^{n_{1}-1}\left(\eta_{r}\right) w_{0}$ at $f^{n_{1}-1}\left(\eta_{r}\right)$. Existence of such an $\tilde{L}$ is precisely the content of the lemma for time $n_{1}$.

A similar argument proves the claim for the subsequent free returns of $z_{r}$. For each $i \geq 1$, let $p_{i} \geq 1$ be maximum such that

$$
\operatorname{dist}\left(f^{n_{i}+j}\left(z_{r}\right), f^{j}\left(\zeta_{n_{i}}\right)\right) \leq e^{-\beta j} \text { for all } 1 \leq j \leq p_{i},
$$

and then let $n_{i+1}$ be the smallest integer $n>n_{i}+p_{i}$ so that $f^{n}\left(z_{r}\right) \in I(\delta)$. We may assume, by induction, that $\left(f^{n_{j}-1}\left(\eta_{r}\right), D f^{n_{j}-1}\left(\eta_{r}\right) w_{0}\right)$ is in tangential position relative to $\left(\zeta_{n_{j}}, t\left(\zeta_{n_{j}}\right)\right)$, for every free return $1 \leq j \leq i$. Then Lemma 3.2 implies,

$$
\left\|D f^{n_{i+1}-1}\left(\eta_{r}\right) w_{0}\right\| \geq \sigma_{1}^{\left(n_{i+1}-1\right) / 5} \geq 2 .
$$

Taking $t\left(\eta_{r}\right)$ in the place of $w_{0}$, we also get (the condition of tangential position, corresponding to hypothesis 1 in Lemma 3.2 , results from Proposition 2.7 )

$$
\left\|D f^{n_{i+1}-1}\left(\eta_{r}\right) t\left(\eta_{r}\right)\right\| \geq \sigma_{1}^{\left(n_{i+1}-1\right) / 5} \geq 2 .
$$

Therefore, the angle between the vectors $D f^{n_{i+1}-1}\left(\eta_{r}\right) w_{0}$ and $D f^{n_{i+1}-1}\left(\eta_{r}\right) t\left(\eta_{r}\right)$ is less than $(C b)^{n_{i+1}-1}$, so it is much smaller than $\operatorname{dist}\left(f^{n_{i+1}-1}\left(\eta_{r}\right), \zeta_{n_{i+1}}\right)$. It follows, as in the previous case, that $\left(f^{n_{i+1}-1}\left(\eta_{r}\right), D f^{n_{i+1}-1}\left(\eta_{r}\right) w_{0}\right)$ is in tangential position to $\left(\zeta_{n_{i+1}}, t\left(\zeta_{n_{i+1}}\right)\right)$, which proves our assertion for $n_{i+1}$. This finishes the proof of Lemma 3.4.

Now let us go back to proving Proposition 3.3. The previous lemma and (20) mean that $z_{r}$ satisfies both assumptions of Lemma 3.2 at all times, and so $\eta_{r}=f\left(z_{r}\right)$ is expanding. We take $\Gamma_{r}$ to be the long stable leaf through $\eta_{r}$ granted by Proposition 2.4. Next we must check that this $\Gamma_{r}$ does satisfy the exponential estimate in (19).

Recall that $f(x, y)=\left(1-a x^{2}, 0\right)+R(a, x, y)$ where the first and the second order derivatives of $R$, with respect to $x, y, a$ are bounded by $C \sqrt{b}$, and $\gamma=\{(x, y(x))\}$ with $\left|y^{\prime}\right|,\left|y^{\prime \prime}\right| \leq C \sqrt{b}$. So, the image $f(\gamma)$ may be written as $\{\xi(x), \eta(x)\}$ with $\xi^{\prime \prime}(x) \approx-2 a \approx-4$ and $\left|\eta^{\prime}\right|,\left|\eta^{\prime \prime}\right| \leq C \sqrt{b}$. On the other hand, by Proposition 2.4 ,

$$
\Gamma^{s}=\left\{\left(x^{s}(y), y\right)\right\} \quad \text { with }\left|\left(x^{s}\right)^{\prime}\right|,\left|\left(x^{s}\right)^{\prime \prime}\right| \leq C \sqrt{b},
$$

and so $\left(x^{s} \circ \eta-\xi\right)^{\prime \prime}(x) \approx-\xi^{\prime \prime}(x) \approx 4$. Write $\zeta_{0}=\left(x_{0}, y_{0}\right)$. Proposition 2.2 .2 implies that $f(\gamma)$ is tangent to $\Gamma^{s}$ at $f\left(\zeta_{0}\right)$, and this gives $\xi\left(x_{0}\right)=x^{s}\left(\eta\left(x_{0}\right)\right)$ and $\xi^{\prime}\left(x_{0}\right)=\left(x^{s} \circ \eta\right)^{\prime}\left(x_{0}\right)$.
Consequently,

$$
3\left(x-x_{0}\right)^{2} \leq x^{s}(\eta(x))-\xi(x) \leq 5\left(x-x_{0}\right)^{2} \quad \text { for every } x .
$$


By construction $z_{r}=\left(x_{r}, y\left(x_{r}\right)\right)$ has $(9 / 10) e^{-r} \leq\left|x_{r}-x_{0}\right| \leq e^{-r}$. So, the horizontal distance $x^{s}\left(\eta\left(x_{r}\right)\right)-\xi\left(x_{r}\right)$ from $z_{r} \in \Gamma_{r}$ to $\Gamma^{s}$ satisfies

$$
2 e^{-2 r} \leq x^{s}\left(\eta\left(x_{r}\right)\right)-\xi\left(x_{r}\right)<5 e^{-2 r} .
$$

Combining this with the Lipschitz estimate in the last part of Proposition 2.4,

$$
\frac{3}{2} e^{-2 r} \leq x^{s}(y)-x_{r}(y)<9 e^{-2 r} \text { for every }|y| \leq 1 / 10 .
$$

where $\left\{\left(x_{r}(y), y\right)\right\}$ represents the long stable leaf $\Gamma_{r}$. This implies (19).

Finally, we prove the proposition for a general critical point $\zeta \in \mathcal{C}$, of generation $g \geq 1$. By Proposition 2.2.3, the segment $\gamma=\gamma(\zeta)$ of radius $\delta \rho^{g}$ around $\zeta$ in $W^{u}(P)$ is a $C^{2}(b)$ curve This means that precisely the same construction of $\Gamma_{r}=\Gamma_{r}(\zeta)$ as in the previous case applies here, for $r$ large enough so that $e^{-r} \leq \delta \rho^{g}$. In particular, we get (24) for all such $r$. On the other hand, for $r<\Delta+g \log (1 / \rho)$ we define $\Gamma_{r}(\zeta)=\Gamma_{r}(\tilde{\zeta})$, where $\tilde{\zeta}$ is any critical point of generation $\tilde{g}<g$ with $\operatorname{dist}(\tilde{\zeta}, \zeta) \leq b^{g / 10}$, as given by Proposition 2.2.3. Proposition 2.4 implies that the horizontal distance between the long stable leaves through the points $f(\zeta)$ and $f(\tilde{\zeta})$ is
bounded by

$$
2 b^{g / 10} \leq 10^{-g}\left(\delta \rho^{g}\right)^{2} \leq 10^{-g} e^{-2 r}
$$

(take $b \ll \delta \ll 1$ ). In view of $(24)$ and the hierarchical form of our construction, the horizontal distance from these $\Gamma_{r}$ to $\Gamma^{s}(f(\zeta))$ satisfies bounds similar to (24), with the factors $3 / 2$ and 9 replaced by 1 and 10 (because $\sum_{g} 10^{-g}<1 / 2$ ). This completes our construction.

Remark 3.3. $\Gamma_{\Delta}$ and $\Gamma_{\Delta+1}$ as constructed above are the same for all critical values (as long as we suppose $\rho<1 / e$, which we clearly can). It is clear that every $\Gamma_{r}(\zeta), \zeta \in \mathcal{C}$ and $r \geq \Delta$, intersects the unstable manifold $W^{u}(P)$, for instance, at the point $\eta_{r}$. Let us also record that, cf. (20) and Lemma $3.4, d_{\mathcal{C}}\left(f^{n-1}\left(\eta_{r}\right)\right) \geq e^{-2 \beta n}$ and $\left(f^{n-1}\left(\eta_{r}\right), D f^{n-1}\left(\eta_{r}\right) w_{0}\right)$ is in tangential position relative to $\left(\zeta_{n}, t\left(\zeta_{n}\right)\right)$, for every free return $n \geq 1$

For the definition of itinerary of an orbit in the basin of $\Lambda$ we need an extended family of long stable leaves $\Gamma_{r, l}=\Gamma_{r, l}(\zeta), \zeta \in \mathcal{C}, r>\Delta, 0 \leq l \leq r^{2}$, with $\Gamma_{r, 0}=\Gamma_{r-1}, \Gamma_{r, r^{2}}=\Gamma_{r}$, and

(a) horiz $\operatorname{dist}\left(\Gamma_{r, l}, W^{s}(f(\zeta))\right) \approx e^{-2 r}$ for every $0 \leq l \leq r^{2}$;

(b) horiz $\operatorname{dist}\left(\Gamma_{r, l-1}, \Gamma_{r, l}\right) \approx e^{-2 r} / r^{2}$ for every $1 \leq l \leq r^{2}$.

Here $\approx$ means equality up to a factor 100 , say. Such a family can be obtained by the following variation of the previous construction. For each $r \geq \Delta+1$, decompose the segment of $W^{u}(P)$ bounded by $z_{r-1}$ and $z_{r}$ into $2 r^{2}-1$ segments of equal length. Denote these segments $\gamma_{r, j}$, for $1 \leq j \leq 2 r^{2}-1$, in such a way that the distance to $z_{r}$ decreases monotonically with $j$. Observe
that

$$
\text { length }\left(\gamma_{r, j}\right) \geq \frac{1}{2 r^{2}-1} \operatorname{dist}\left(z_{r}, z_{r+1}\right) \geq \frac{1}{2 r^{2}} \frac{1}{2} e^{-r},
$$

for every $j$. Given any $z \in \gamma_{r, j}$, we have $d_{\mathcal{C}}(z) \leq e^{-r}$, and so

$$
2 c_{1} \frac{d_{\mathcal{C}}(z)}{\left|\log d_{\mathcal{C}}(z)\right|^{2}} \leq 2 c_{1} \frac{e^{-r}}{r^{2}} \leq \frac{e^{-r}}{4 r^{2}} \leq \operatorname{length}\left(\gamma_{r, j}\right)
$$


(we took $c_{1}<1 / 10$ ). This means that every segment $\gamma_{r, j}$ satisfies the condition in Remark 3.1, and so it contains a point $z_{r, j}$ such that $f\left(z_{r, j}\right)$ is expanding. We let $\Gamma_{r, l}=\Gamma_{r, l}(\zeta)$ be the long stable leaf through the point $f\left(z_{r, 2 l}\right)$, for each $1 \leq l \leq r^{2}-1$. The estimates in (a) and (b) follow from the same arguments as we used to prove (19). Remark 3.3 renains valid for this extended family $\Gamma_{r, l}$.

\subsection{Itineraries for orbits in the basin}

To each point $z \in B(\Lambda)$ we want to associate sequences $n_{j}, i_{j}=\left(\tilde{\zeta}_{j}, r_{j}, l_{j}, \epsilon_{j}\right), j \geq 0$, where $n_{j}$ is an integer, $\tilde{\zeta}_{j} \in \mathcal{C},\left(r_{j}, l_{j}\right) \in\{(0,0)\} \cup\left\{(r, l): r \geq \Delta\right.$ and $\left.1 \leq l \leq r^{2}\right\}$, and $\epsilon_{j} \in\{+, 0,-\}$. Roughly, one may think of $n_{j}$ as the $j$ th free return of $z$, and of $\bar{\zeta}_{j}$ as the corresponding binding point; $r_{j}, l_{j}, \epsilon_{j}$ describe the position of $f^{n_{j}+1}(z)$ relative to the long stable leaves $\Gamma_{r, l}\left(\tilde{\zeta}_{j}\right)$. The precise construction of these sequences occupies the whole of this section.

Recall that $G_{0}, G_{1}$ contain long $C^{2}(b)$ segments $\gamma_{0}, \gamma_{1}$, around the critical points $\zeta_{0}, \zeta_{1}$, respectively. In view of the form of our map, for each $i=0,1$ we may write $f\left(\gamma_{i}\right)$ as $\left\{\xi_{i}(x), \eta_{i}(x)\right\}$ with $\xi_{i}^{\prime \prime} \approx-2 a \approx 4$ and $\left|\eta_{i}\right|,\left|\eta_{i}^{\prime}\right|,\left|\eta_{i}^{\prime \prime}\right| \leq C \sqrt{b}$. In particular, $f\left(\gamma_{i}\right)$ intersects each $\Gamma_{r, l}\left(\zeta_{i}\right)$, for $0 \leq l \leq r^{2}$, in exactly two points. Let $\Delta_{i}$ be the region bounded by $f\left(\gamma_{i}\right)$ and by the long stable leaf $W_{\text {loc }}^{s}(P)$ passing through $P$, see Figure 1. Since $f\left(\gamma_{0}\right)$ and $f\left(\gamma_{1}\right)$ are disjoint, whereas $\Delta_{0}$ and $\Delta_{1}$ must intersect each other (e.g. extend $\left\{\gamma_{0}, \gamma_{1}\right\}$ to a foliation by nearly horizontal curves, and use that the image of each leaf intersects every vertical line in not more than two points), we have either $\Delta_{1} \subset \Delta_{0}$ or $\Delta_{0} \subset \Delta_{1}$.

We consider $\Delta_{1} \subset \Delta_{0}$, as the other case is analogous. In the sequel we define $n_{j}(z), i_{j}(z)$, $j \geq 0$, for points $z \in \Delta_{0}$. The extension to generic points $w \in B(\Lambda)$ is, simply, by taking $n_{j}(w)=n+n_{j}\left(f^{n}(w)\right)$ and $i_{j}(w)=i_{j}\left(f^{n}(w)\right)$ for each $j \geq 0$, where $n \geq 0$ is the smallest integer for which $f^{n}(w) \in \Delta_{0}$. Since Lebesgue almost every point in the basin of $\Lambda$ has some iterate contained in $\Delta_{0}$, cf. Section 5 , this leaves out only a zero Lebesgue measure subset of $B(\Lambda)$, which is negligible for our purposes.

Before proceeding, let us make a few simple conventions. In what follows $(r, l)$ should be replaced by $\left(r-1,(r-1)^{2}+l\right)$ if $l \leq 0$, and by $\left(r+1, l-r^{2}\right)$ if $l>r^{2}$. We say that $\left(r_{1}, l_{1}\right)>\left(r_{2}, l_{2}\right)$ if either $r_{1}>r_{2}$ or $r_{1}=r_{2}$ and $l_{1}>l_{2}$. The region in between two long stable leaves $\Gamma_{1}=\left\{\left(x_{1}(y), y\right):|y| \leq 1 / 10\right\}$ and $\Gamma_{2}=\left\{\left(x_{2}(y), y\right):\{y \mid \leq 1 / 10\}\right.$, with $x_{1}<x_{2}$, is $\left\{(x, y): x_{1}(y)<x \leq x_{2}(y),|y| \leq 1 / 10\right\}$.

Let $(\hat{r}, \hat{l})$ be defined by the condition that $f\left(\zeta_{1}\right)$ is in the region of $\Delta_{0}$ in between $\Gamma_{\hat{r}, \hat{l}}\left(\zeta_{0}\right)$ and $\Gamma_{\hat{r}, \hat{l}-1}\left(\zeta_{0}\right)$. For $z \in \Delta_{0}$ we define $n_{0}=-1$ and

(a) $i_{0}(z)=\left(\zeta_{0}, r, l, 0\right)$ if $z$ is in the region of $\Delta_{0}$ in between $\Gamma_{r, l}\left(\zeta_{0}\right)$ and $\Gamma_{r, l-1}\left(\zeta_{0}\right)$, with $(r, l)>(\hat{r}, \hat{l})$

(b) $i_{0}(z)=\left(\zeta_{0}, \hat{r}, \hat{l}+1,0\right)$ if $z$ is in the region of $\Delta_{0}$ in between $W_{l o c}^{s}\left(f\left(\zeta_{1}\right)\right)$ and $\Gamma_{\hat{r}, \hat{l}}\left(\zeta_{0}\right)$;

(c) $i_{0}(z)=\left(\zeta_{1}, r, l, \pm\right)$ if $z$ is in either of the two regions of $\Delta_{0} \backslash \Delta_{1}$ in between $\Gamma_{r, l}\left(\zeta_{1}\right)$ and $\Gamma_{r, l-1}\left(\zeta_{1}\right)$, the sign $+/-$ corresponding to the upper/lower region;

(d) $i_{0}(z)=\left(\zeta_{1}, 0,0, \pm\right)$ if $z$ is in either of the two regions of $\Delta_{0} \backslash \Delta_{1}$ in between $\Gamma_{\Delta}$ and $W_{\text {loc }}^{s}(P)$, the sign $+/-$ corresponding to the upper/lower region; 


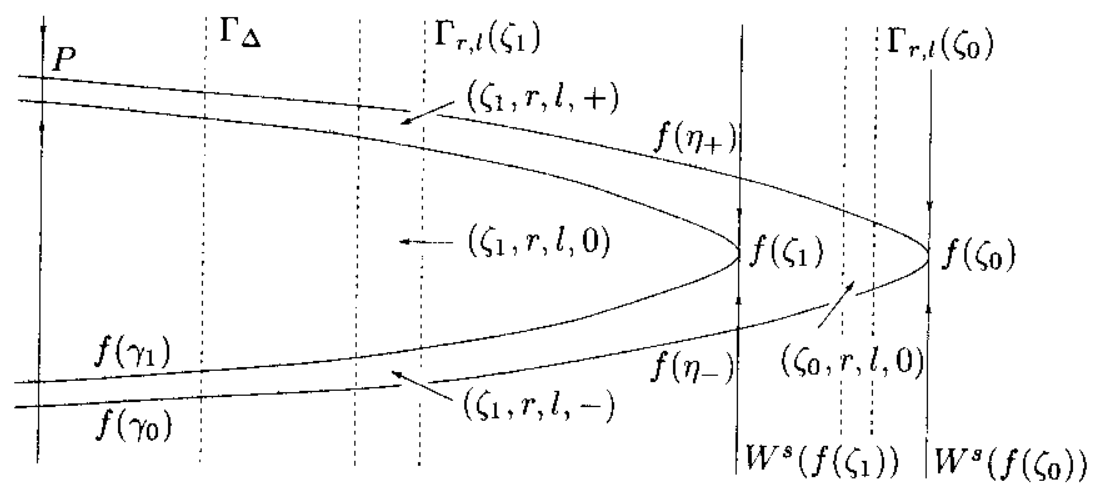

Figure 1:

(e) $i_{0}(z)=\left(\zeta_{1}, r, l, 0\right)$ if $z$ is in the region of $\Delta_{1}$ in between $\Gamma_{r, l}\left(\zeta_{1}\right)$ and $\Gamma_{r, l-1}\left(\zeta_{1}\right)$.

(f) $i_{0}(z)=\left(\zeta_{1}, 0,0,0\right)$ if $z$ is in the region of $\Delta_{1}$ in between $\Gamma_{\Delta}$ and $W_{l o c}^{s}(P)$.

We also define $R\left(i_{0}\right)=\left\{z \in \Delta: i_{0}(z)=i_{0}\right\}$ for each $i_{0}=\left(\tilde{\zeta}_{0}, r_{0}, l_{0}, \epsilon_{0}\right)$ as before. This closes the first step of our definition.

The definition of these objects proceeds by recurrence. In the next paragraphs we explain how $n_{1}(z)$ and $i_{1}(z)$ are defined for $z$ in $R\left(i_{0}\right)$, for each fixed $i_{0}$

In cases $(\mathrm{a}),(\mathrm{b}),(\mathrm{c}),(\mathrm{e})$, define $p_{1}=p_{1}\left(i_{0}\right) \geq 1$ to be the largest integer such that

$$
\operatorname{dist}\left(f^{j-1}(z), f^{j}\left(\tilde{\zeta}_{0}\right)\right) \leq e^{-\beta j} \text { for } 1 \leq j \leq p_{1} \text { and every } z \in R\left(i_{0}\right) .
$$

For (d), (f) just set $p_{1}=0$. In any case, let $m_{1}=n_{1}>p_{1}$ be minimum such that $f^{n_{1}}\left(R\left(i_{0}\right)\right)$ intersects $I(\delta)$. Denote $\gamma_{0}^{u}, \gamma_{1}^{u}, \gamma_{0}^{s}, \gamma_{1}^{s}$ the four segments forming the boundary of the rectangle $R\left(i_{0}\right)$, with $\gamma_{i}^{u} \subset W^{u}(P)$ and $\gamma_{i}^{s}$ contained in long stable leaves, $i=0,1$. The following lemma will be proved in Section 3.3.

Lemma 3.5. 1. $m_{1}>p_{1} \geq r_{0}$ and

2. the slope of $f^{n_{1}}\left(\gamma_{i}^{u}\right)$ is less than $C \sqrt{b}$ at every point;

3. length $\left(f^{n_{1}}\left(\gamma_{j}^{s}\right)\right) \leq(1 / 10) d_{\mathcal{C}}\left(z_{i}^{*}\right)$;

4. angle $\left(t\left(z_{0}^{*}\right), t\left(z_{1}^{*}\right)\right) \leq(1 / 10) d_{\mathcal{C}}\left(z_{i}^{*}\right)$;

for $i=0,1$ and $j=0,1$, where $z_{0}^{*}, z_{1}^{*}$ are the endpoints of $f^{n i}\left(\gamma_{j}^{s}\right)$.

By Proposition 2.7, the free segments $f^{n_{1}}\left(\gamma_{i}^{u}\right)$ can be extended to $C^{2}$ curves $K_{i}=\left\{\left(x, y_{i}(x)\right\}\right.$ with $\left|y_{i}^{\prime}\right|,\left|y_{i}^{\prime \prime}\right| \leq 1 / 10$, tangent to $W^{u}(P)$ at some critical point $\eta_{i} \in K_{i}$. By definition, $d_{\mathcal{C}}\left(z_{i}^{*}\right)=$ $\operatorname{dist}\left(z_{i}^{*}, \eta_{i}\right)$. Recall that $\eta_{i}$ need not belong to $f^{n_{1}}\left(\gamma_{i}^{u}\right)$. We can also not discard the possibility that $\eta_{0}=\eta_{1}$. On the other hand, according to the next lemma, either both $\eta_{i}$ belong to the corresponding $f^{n_{1}}\left(\gamma_{i}^{u}\right)$ or none does, and in the latter case we may always take the two critical points to coincide. 


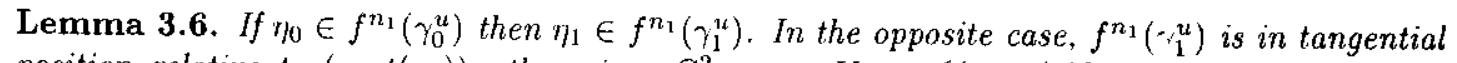
position relative to $\left(\eta_{0}, t\left(\eta_{0}\right)\right)$ : there is a $C^{2}$ curve $K_{2}=\left\{\left(x, y_{2}(x)\right\}\right.$ with $\left|y_{2}^{\prime}\right|,\left|y_{2}^{\prime \prime}\right| \leq 1 / 5$, containing $f^{n_{1}}\left(\gamma_{1}^{u}\right)$ and tangent to $W^{u}(P)$ at $\eta_{0}$.

Proof. Suppose that $\eta_{0} \in f^{n_{1}}\left(\gamma_{0}^{u}\right)$ but $\eta_{1} \in K_{1} \backslash f^{n_{1}}\left(\gamma_{1}^{u}\right)$. Let $z_{1}^{*}$ be the boundary point of $f^{n_{1}}\left(\gamma_{1}^{u}\right)$ closest to $\eta_{1}$, and $z_{0}^{*}$ be the boundary point of $f^{n_{1}}\left(\gamma_{0}^{u}\right)$ that is in the same stable leaf $f^{n_{1}}\left(\gamma_{j}^{s}\right)$ as $z_{1}^{*}$. Let $m \geq 1$ be fixed such that

$$
\tau^{m+1}<5 \max \left\{d_{\mathcal{C}}\left(z_{i}^{*}\right): i=0,1\right\} \leq \tau^{m},
$$

where $\tau>0$ is taken as in Proposition 2.3. Let $\left[\eta_{i}, z_{i}^{*}\right]$ denote the segment of $K_{i}$ connecting $\eta_{i}$ to $z_{i}^{*}$. Then the contractive direction of order $m$ is well defined at every point of $f\left(\left[\eta_{i}, z_{i}^{*}\right]\right)$. We write $f^{n_{1}}\left(\gamma_{i}^{u}\right)=\left\{\left(s, y_{i}(s)\right)\right\}$ and $\left(\xi_{i}(s), \eta_{i}(s)\right)=f\left(s, y_{i}(s)\right)=\left(1-a s^{2}, 0\right)+R\left(a, s, y_{i}(s)\right)$, and split the tangent vector

$$
\left(\xi_{i}^{\prime}(s), \eta_{i}^{\prime}(s)\right)=\alpha_{i}(s) e_{i}(s)+\beta_{i}(s) w_{0} .
$$

Here $e_{i}(s)$ is the contracting direction of order $m$ at the point $\left(\xi_{i}(s), \eta_{i}(s)\right)$, recall that it may be represented by a vector $\left(\epsilon_{i}, 1\right)$ with $\left\|\epsilon_{i}\right\|_{C^{1}} \leq C \sqrt{b}$. Then $\beta_{i}(s)=\xi_{i}^{\prime}(s)-\epsilon_{i}(s) \eta_{i}^{\prime}(s)$ and, using $\|R\|_{C^{2}} \leq C \sqrt{b}$ and Lemma 3.5 ,

$$
\left|\beta_{1}\left(z_{1}^{*}\right)-\beta_{0}\left(z_{0}^{*}\right)\right| \leq(2 a+C \sqrt{b}) \operatorname{dist}\left(z_{0}^{*}, z_{1}^{*}\right)+C \sqrt{b} \operatorname{angle}\left(t\left(z_{0}^{*}\right), t\left(z_{1}^{*}\right)\right) \leq \min \left\{d_{\mathcal{C}}\left(z_{0}^{*}\right), d_{\mathcal{C}}\left(z_{1}^{*}\right)\right\}
$$

Now, suppose that $\eta_{1}$ is to the right of $z_{1}^{*}$ in $K_{1}$ (and $\eta_{0}$ is to the left of $z_{0}^{*}$ in $K_{0}$ ); the opposite case is entirely analogous. Then, as in (7),

$$
\beta_{1}\left(z_{1}^{*}\right)-\beta_{1}\left(\eta_{1}\right) \approx 2 a d_{\mathcal{C}}\left(z_{1}^{*}\right) \geq 3 d_{\mathcal{C}}\left(z_{1}^{*}\right) \text { and } \beta_{0}\left(\eta_{0}\right)-\beta_{0}\left(z_{0}^{*}\right) \approx 2 a d_{\mathcal{C}}\left(z_{0}^{*}\right) \geq 3 d_{\mathcal{C}}\left(z_{0}^{*}\right) .
$$

Moreover, $\left|\beta_{i}\left(\eta_{i}\right)\right| \leq(C b)^{m}$ because of Proposition 2.3 .2 and the fact that $\eta_{i}$ is a critical point, recall Theorem 2.1.2. Since $(C b)^{m}$ is much smaller than $\tau^{m} \approx \max \left\{d_{\mathcal{C}}\left(z_{i}^{*}\right): i=0,1\right\}$, we get

$$
\beta_{1}\left(z_{1}^{*}\right)-\beta_{0}\left(z_{0}^{*}\right) \geq 2 d_{\mathcal{C}}\left(z_{0}^{*}\right)+2 d_{\mathcal{C}}\left(z_{1}^{*}\right) .
$$

We have reached a contradiction, and so we must have $\eta_{1} \in f^{n_{1}}\left(\gamma_{1}^{u}\right)$.

To prove the second part, let $z_{0}^{*}$ be the boundary point of $f^{n_{1}}\left(\gamma_{0}^{u}\right)$ closest to $\eta_{0}$ in $K_{0}$, and $z_{1}^{*}$ be the boundary point of $f^{n_{1}}\left(\gamma_{1}^{u}\right)$ in the same stable leaf as $z_{0}^{*}$. By parts 3 and 4 of Lemma 3.5 , both $\operatorname{dist}\left(z_{0}^{*}, z_{1}^{*}\right)$ and angle $\left(t\left(z_{0}^{*}\right), t\left(z_{1}^{*}\right)\right)$ are smaller than $(1 / 10) \operatorname{dist}\left(z_{0}^{*}, \eta_{0}\right)$. So, we may modify $K_{0}=\left\{\left(x, y_{0}(x)\right)\right\}$ to get a curve $K_{2}$ as in the statement.

We define $i_{1}(z)$ first when $\eta_{i} \in f^{n_{1}}\left(\gamma_{i}^{u}\right)$ for $i=0,1$. Up to interchanging subscripts, we may suppose that $f\left(\eta_{0}\right)$ is to the right of $f\left(\eta_{1}\right)$, meaning that its long stable leaf is to the right of the one passing through $f\left(\eta_{1}\right)$. Then $f\left(\eta_{1}\right)$ is contained in a region bounded by $f^{n_{1}+1}\left(\gamma_{0}^{u}\right)$ and some pair of long leaves $\Gamma_{\hat{r}, i}\left(\eta_{0}\right), \Gamma_{\hat{r}, \hat{l}+1}\left(\eta_{0}\right)$. We let, see Figure 2 ,

(a1) $i_{1}(z)=\left(\eta_{0}, r, l, 0\right)$ if $f^{n_{1}}(z)$ is in the region of $f^{n_{1}}\left(R\left(i_{0}\right)\right)$ in between $\Gamma_{r, l}\left(\eta_{0}\right)$ and $\Gamma_{r, l-1}\left(\eta_{0}\right)$, with $(r, l)>(\hat{r}, \hat{l})$

(b1) $i_{1}(z)=\left(\eta_{0}, \hat{r}, \hat{l}+1,0\right)$ if $f^{n_{1}}(z)$ is in the region of $f^{n_{1}}\left(R\left(i_{0}\right)\right)$ in between $W_{l o c}^{s}\left(f\left(\eta_{1}\right)\right)$ and $\Gamma_{\hat{r}, i}\left(\zeta_{0}\right) ;$ 
(c1) $i_{1}(z)=\left(\eta_{1}, r, l, \pm\right)$ if $f^{n_{1}}(z)$ is in either of the regions of $f^{n_{1}}\left(R\left(i_{0}\right)\right)$ in between $\Gamma_{r, l}\left(\eta_{1}\right)$ and $\Gamma_{r, l-1}\left(\eta_{1}\right)$, the sign $+/-$ corresponding to the upper/lower region.

(d1) $i_{1}(z)=\left(\eta_{1}, 0,0, \pm\right)$ if $f^{n_{1}}(z)$ is in either of the regions of $f^{n_{1}}\left(R\left(i_{0}\right)\right)$ to the left of $\Gamma_{\Delta}$, the sign $+/-$ corresponding to the upper/lower region.

It is worth keeping in mind that the stable leaves $f^{n_{1}+1}\left(\gamma_{i}^{s}\right)$ on the boundary of $f^{n_{3}+1}\left(R\left(i_{0}\right)\right)$ can not intersect a long leaf unless they are totally contained in it.

The definition of $i_{1}(z)$ is slightly simpler in the case when $\eta_{i} \notin f^{n_{1}}\left(\gamma_{i}^{u}\right)$ for $i=0,1$. Taking advantage of the fact that both segments $f^{n_{1}}\left(\gamma_{i}^{u}\right), i=0,1$, are in tangential position relative to $\eta_{0}$, cf. Lemma 3.6, we define

(a2) $i_{1}(z)=\left(\eta_{0}, r, l,+\right)$ if $f^{n_{1}}(z)$ is in the region of $f^{n_{1}}\left(R\left(i_{0}\right)\right)$ in between $\Gamma_{r, l}\left(\eta_{0}\right)$ and
$\Gamma_{r, l-1}\left(\eta_{0}\right)$ (b2) $i_{1}(z)=\left(\eta_{0}, 0,0,+\right)$ if $f^{n_{1}}(z)$ is in the region of $f^{n_{1}}\left(R\left(i_{0}\right)\right)$ to the left of $\Gamma_{\Delta}$.

See Figure 2.

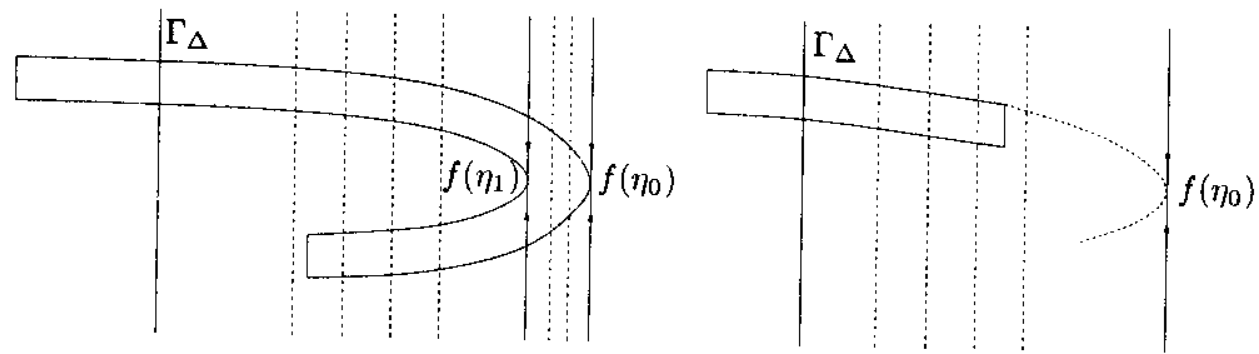

Figure 2:

This completes the definition of $i_{1}(z)$. We also set $R\left(i_{0}, i_{1}\right)=\left\{z \in R\left(i_{0}\right): i_{1}(z)=i_{1}\right\}$, for each $i_{0}=\left(\bar{\zeta}_{0}, r_{0}, l_{0}, \epsilon_{0}\right)$ and $i_{1}=\left(\tilde{\zeta}_{1}, r_{1}, l_{1}, \epsilon_{1}\right)$.

Finally, we define $i_{k}(z)$ for general $k \geq 1$. This is very similar to the case $k=1$, and so we go more quickly now. Suppose $i_{j}(z), n_{j}(z)$, and $R\left(i_{0}, \ldots, i_{j}\right)$ have been defined for every $j<k$. Let $i_{j}=\left(\tilde{\zeta}_{j}, r_{j}, l_{j}, \epsilon_{j}\right), j=0, \ldots, k-1$, be fixed, and $z \in R\left(i_{0}, \ldots, i_{k-1}\right)$.

If $r_{k-1} \geq \Delta$ then we define $p_{k}=p_{k}\left(i_{0}, \ldots, i_{k-1}\right) \geq 1$ to be the largest integer such that

$$
\operatorname{dist}\left(f^{j}(\zeta), f^{j}\left(\tilde{\zeta}_{k-1}\right)\right) \leq e^{-\beta_{j}} \text { for } 1 \leq j \leq p_{k} \text { and every } \zeta \in f^{n_{k-1}}\left(R\left(i_{0}, \ldots, i_{k-1}\right)\right) .
$$

If $r_{k-1}=0$, we just set $p_{k}=0$. Then we let $n_{k}$ be the smallest integer larger than $n_{k-1}+p_{k}$ such that $f^{n_{k}}\left(R\left(i_{0}, \ldots, i_{k-1}\right)\right)$ intersects $I(\delta)$, and let $m_{k}=n_{k}-\left(n_{k-1}+1\right)$. Call $\gamma_{0}^{u}, \gamma_{1}^{u}, \gamma_{0}^{s}, \gamma_{1}^{s}$ the four segments in the boundary of $f^{n_{k-1}+1}\left(R\left(i_{0}, \ldots, i_{k-1}\right)\right)$, with $\gamma_{i}^{u} \subset W^{u}(P)$ and $\gamma_{i}^{s}$ contained in long stable leaves. Then,

Lemma 3.7. 1. $m_{k}>p_{k} \geq r_{k-1}$ and

2. the slope of $f^{m_{k}}\left(\gamma_{i}^{u}\right)$ is less than $C \sqrt{b}$ at every point; 
3. length $f^{m_{k}}\left(\gamma_{j}^{s}\right) \leq(1 / 10) d_{\mathcal{C}}\left(z_{i}^{*}\right) ;$

4. angle $\left(t\left(z_{0}^{*}\right), t\left(z_{1}^{*}\right)\right) \leq(1 / 10) d_{\mathcal{C}}\left(z_{i}^{*}\right) ;$

for $i=0,1$ and $j=0,1$, where $z_{0}^{*}, z_{1}^{*}$ are the endpoints of $f^{m_{k}}\left(\gamma_{j}^{s}\right)$.

This lemma will be proved in Section 3.3. Combining it with Proposition 2.7 we get the analog of Lemma 3.6 at every return:

Lemma 3.8. Either there are two critical points $\eta_{0}, \eta_{1}$ such that $\eta_{i} \in f^{m_{k}}\left(\gamma_{i}^{u}\right)$ for $i=0$ and $i=1$, or there is a critical point $\eta_{0}$ such that both segments $f^{m_{k}}\left(\gamma_{i}^{u}\right), i=0,1$, are in tangential position relative to $\left(\eta_{0}, t\left(\eta_{0}\right)\right)$.

In the first case we define $\hat{r}, \hat{l}$ just as before. Then we let $i_{k}(z)$ be given by the rules which are obtained replacing $f^{n_{1}}(z)$ by $f^{n_{k}}(z)$, and $f^{n_{1}}\left(R\left(i_{0}\right)\right)$ by $f^{n_{k}}\left(R\left(i_{0}, \ldots, i_{k-1}\right)\right)$ in (a1)-(d1). In the second case in the lemma we define $i_{k}(z)$ by the rules obtained by making the corresponding substitutions in (a2)-(b2). Finally, for each $i_{0}, \ldots, i_{k-1}, i_{k}$,

$$
R\left(i_{0}, \ldots, i_{k-1}, i_{k}\right)=\left\{z \in R\left(i_{0}, \ldots, i_{k-1}\right): i_{k}(z)=i_{k}\right\} .
$$

Our definition of itinerary of a point $z$ in the basin of $\Lambda$ is complete. By construction, every $R\left(i_{0}, \ldots, i_{k}\right)$ is a rectangle. Note that the two segments of unstable manifold on its boundary are also contained in the boundary of $R\left(i_{0}, \ldots, i_{k-1}\right)$.

\subsection{Geometry of rectangles at return times}

Here we prove Lemmas 3.5 and 3.7. Beforehand, we derive two other facts concerning the size and shape of rectangles $f^{n_{k}}\left(R\left(i_{0}, \ldots, i_{k-1}\right)\right)$, stated in Lemmas 3.9 and 3.11 below. We call unstable sides of a rectangle the segments of unstable manifold on its boundary, and unstable boundary the union of the unstable sides. Stable sides and stable boundary are defined analogously.

Lemma 3.9. Given $z \in R\left(i_{0}, \ldots, i_{k-1}\right)$ and any unstable side $\sigma^{u}$ of $R\left(i_{0}, \ldots, i_{k-1}\right)$, there exists $\xi_{k} \in \sigma^{u}$ such that

$$
\operatorname{dist}\left(f^{n_{k}}(z), f^{n_{k}}\left(\xi_{k}\right)\right) \leq(C b)^{n_{k} / 2} .
$$

If $z$ is in some stable side $\sigma^{s}$, we may take $\xi_{k}$ the common endpoint of $\sigma^{s}$ and $\sigma^{u}$.

Proof. To keep track of the constant $C$ in the statement, we denote it $C_{h}$ throughout the proof. We shall take $C_{h} \geq 100 C_{1}$, where $C_{1}$ is the constant $C$ in Proposition 2.3.

We start by proving the lemma for $k=1$. As a consequence of Propositions 2.8.4 and 2.5, every $z \in R\left(i_{0}\right)$ is expanding up to time $m_{1}=n_{1}$. So, using Proposition 2.3 in the same way as when proving Proposition 2.4 in Section 2, the temporary stable leaf of order $n_{1}$ through $z$ is a long nearly vertical curve: $\Gamma^{n_{1}}(z)=\{(x(y), y):|y| \leq 1 / 10\}$ with $\left|x^{\prime}\right|,\left|x^{\prime \prime}\right| \leq C \sqrt{b}$, and

$$
\operatorname{dist}\left(f^{j}(\xi), f^{j}(z)\right) \leq(C b)^{j} \operatorname{dist}(\xi, z) \text { for all } \xi \in \Gamma^{n_{1}}(z) \text { and } 1 \leq j \leq n_{1} .
$$

If $\Gamma^{n_{1}}(z)$ intersects the unstable segment $\sigma^{u}$, take $\xi_{1}$ to be the intersection point. Then $\operatorname{dist}\left(f^{n_{1}}(z), f^{n_{1}}\left(\xi_{1}\right)\right) \leq\left(C_{1} b\right)^{n_{1}}$, which is even stronger than the claim. If $\Gamma^{n_{1}}(z)$ leaves the 

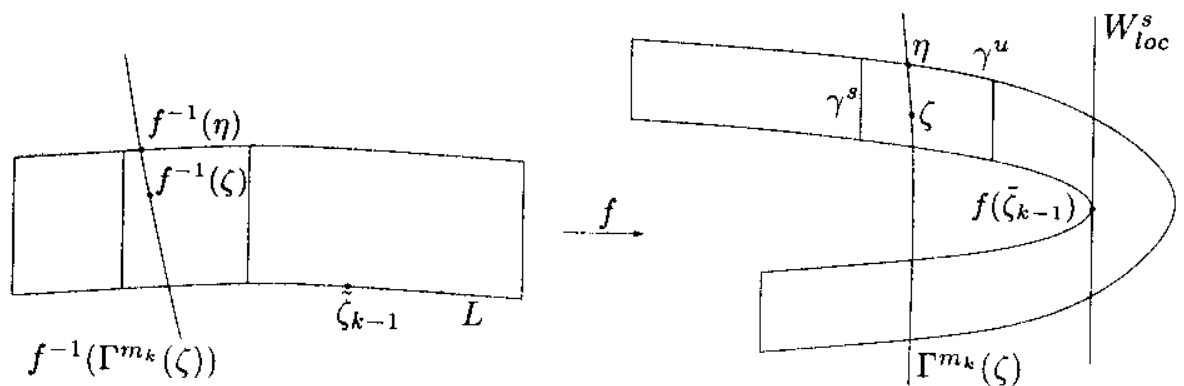

Figure 3:

rectangle $R\left(i_{0}\right)$ through a stable leaf $\sigma^{s}$, the argument has to be slightly modified. We take $\xi_{1}$ to be the vertex of $R\left(i_{0}\right)$ where $\sigma^{s}$ meets $\sigma^{u}$, then continue as before (noting that $\sigma^{s}$ is also contracted by positive iterates of $f$ ).

Now we assiume that the lemma is true at time $n_{k-1}$, and prove that it must be true also at time $n_{k}$. For the same reasons as before, every point $\zeta=f^{n_{k-1}+1}(z), z \in R\left(i_{0}, \ldots, i_{k-1}\right)$, is expanding up to time $m_{k}$. So, the temporary stable leaf $\Gamma^{m_{k}}(\zeta)$ of order $m_{k}$ through $\zeta$ is a long nearly vertical curve. If $\Gamma^{m_{k}}(\zeta)$ crosses the unstable segment $\gamma^{u}=f^{n_{k-1}+1}\left(\sigma^{u}\right)$, let $\eta$ be the intersection point. See Figure 3. Otherwise, $\Gamma^{m_{k}}(\zeta)$ intersects some stable side $\gamma^{s}$ of $f^{n_{k-1}+1}\left(R\left(i_{0}, \ldots, i_{k-1}\right)\right)$, and we call $\eta$ the common endpoint of $\gamma^{u}$ and $\gamma^{s}$. Either way, define $\xi_{k}$ by $f^{n_{k-1}+1}\left(\xi_{k}\right)=\eta$. The conclusion of the lemma for $k>1$ is an easy consequence of the following result, whose proof we postpone for a while:

Lemma 3.10. Assume Lemma 3.9 is true for $k-1$. For $\eta$ and $\zeta$ as above,

$$
\operatorname{dist}(\zeta, \eta) \leq 10\left(C_{h} b\right)^{n_{k-1} / 2} e^{r_{k-1}} \quad \text { and } \quad \operatorname{dist}(\zeta, \eta) \leq 10\left(C_{h} b\right)^{n_{k-1} / 4} .
$$

Indeed, combining the first of these inequalities with Proposition 2.3.2,

$$
\operatorname{dist}\left(f^{n_{k}}(z), f^{n_{k}}\left(\xi_{k}\right)\right)=\operatorname{dist}\left(f^{m_{k}}(\zeta), f^{m_{k}}(\eta)\right) \leq\left(C_{1} b\right)^{m_{k}} 10\left(C_{h} b\right)^{n_{k-1} / 2} e^{r_{k-1}} \leq\left(C_{h} b\right)^{n_{k} / 2} .
$$

since $n_{k}=n_{k-1}+1+m_{k}, m_{k}>r_{k-1}$, and we chose $C_{h} \geq 100 C_{1}$. This gives Lemma 3.9 .

Now we prove Lemma 3.10 :

Proof. It is convenient to distinguish two cases, depending on the relative size of $r_{k-1}$ and $n_{k-1}$. We treat first the case when $r_{k-1}$ is small with respect to $n_{k-1}$ :

$$
\left(C_{h} b\right)^{n_{k-1}} \leq e^{-4 r_{k-1}}
$$

Note that in this case, the second inequality in the statement of the lemma is a direct consequence of the first one.

To prove the first inequality, consider a curve $L=\left\{\left(x, y_{0}(x)\right)\right\}$ with $\left|y_{0}^{\prime}\right|,\left|y_{0}^{\prime \prime}\right| \leq 1 / 5$, tangent to $W^{u}(P)$ at $\tilde{\zeta}_{k-1}=(\bar{x}, \bar{y})$ and containing one of the unstable sides of $f^{n_{k-1}}\left(R\left(i_{0}, \ldots, i_{k-2}\right)\right)$. 
We claim that a segment of $f^{-1}\left(\Gamma^{m_{k}}(\zeta)\right)$ can be parametrised $t \mapsto z(t)=\left(x(t), y_{0}(x(t))+t\right)$, with

$$
\left|x^{\prime}(t)\right| \leq C \sqrt{b} e^{r_{k-1}} \quad \text { and } \quad|t| \leq 2\left(C_{h} b\right)^{n_{k-1} / 2} .
$$

Since $L$ is nearly horizontal, $\operatorname{dist}(z(t), L) \geq|t| / 2$. So, Lemma 3.9 for $k-1$ implies that $z(t)$ has to cross the boundary of $f^{n_{k-1}}\left(R\left(i_{0}, \ldots, i_{k-1}\right)\right)$ at some $t=t_{2}$ with $0<\left|t_{2}\right| \leq 2\left(C_{h} b\right)^{n_{k-1} / 2}$. Moreover, $f^{-1}(\zeta)=z\left(t_{1}\right)$ for some $t_{1}$ in between zero and $t_{2}$. Typically, $z\left(t_{2}\right)=f^{-1}(\eta)$ but it may also happen that $z\left(t_{2}\right)$ be in a stable side $\gamma^{s}$ of $f^{n_{k-1}}\left(R\left(i_{0}, \ldots, i_{k-1}\right)\right)$, with $f^{-1}(\eta)$ being an endpoint of $\gamma^{s}$. In the second case, $\operatorname{dist}\left(z\left(t_{2}\right), f^{-1}(\eta)\right)$ is less than $\left(C_{h} b\right)^{n_{k-1} / 2}$, by the induction hypothesis. So, we always have, using the bound for $\left|x^{\prime}\right|$ given in (25),

$$
\begin{aligned}
\operatorname{dist}(\zeta, \eta) & \leq \operatorname{dist}\left(z\left(t_{1}\right), z\left(t_{2}\right)\right)+\left(C_{h} b\right)^{n_{k-1} / 2} \\
& \leq C \sqrt{b} e^{r_{k-1}}\left(C_{h} b\right)^{n_{k-1} / 2}+5\left(C_{h} b\right)^{n_{k-1} / 2} \leq 10 e^{r_{k-1}}\left(C_{h} b\right)^{n_{k-1} / 2} .
\end{aligned}
$$

To turn the previous paragraph into a complete proof of the lemma for small $r_{k-1}$, we have to justify the claims in (25). Let $\tilde{\zeta}_{k-1}=(\bar{x}, \bar{y})$ be the binding critical point. Write the long leaf $W_{\text {loc }}^{s}\left(f\left(\tilde{\zeta}_{k-1}\right)\right)$ through $f\left(\tilde{\zeta}_{k-1}\right)=\left(\bar{x}_{1}, \bar{y}_{1}\right)$ as $\left\{\left(x^{s}(y), y\right):|y| \leq 1 / 10\right\}$, and the temporary stable leaf $\Gamma^{m_{k}}(\zeta)$ as $\left\{\left(x_{\zeta}(y), y\right):|y| \leq 1 / 10\right\}$. By Proposition 2.3.2 and 2.3.3,

$$
\left|\left(x^{s}-x_{\zeta}\right)^{\prime}(y)\right| \leq C \sqrt{b}\left(x^{s}-x_{\zeta}\right)(y)+(C b)^{m_{k}} .
$$

Combining this with $m_{k}>r_{k-1}$, cf. Lemma 3.7.1, and the definition of $r_{k-1}$,

$$
\frac{1}{2} e^{-2 r_{k-1}} \leq\left(x^{s}-x_{\zeta}\right)(y) \leq 20 e^{-2 r_{b-1}} \quad \text { and } \quad\left|\left(x^{s}-x_{\zeta}\right)^{\prime}(y)\right| \leq C \sqrt{b} e^{-2 r_{k-1}}
$$

for every $|y| \leq 1 / 10$ (compare Proposition 3.3). Next, we write $f(L)=\left\{\left(X_{0}(x), Y_{0}(x)\right)\right\}$ with $X_{0}^{\prime \prime} \approx-2 a \approx \overline{4}$ and $\left|Y_{0}^{\prime}\right|,\left|Y_{0}^{\prime \prime}\right| \leq C \sqrt{b}$. Since $f(L)$ is tangent to $W_{\text {loc }}^{s}\left(f\left(\tilde{\zeta}_{k-1}\right)\right)$ at $f\left(\tilde{\zeta}_{k-1}\right)$,

$$
\left(x^{s} \circ Y_{0}-X_{0}\right)(x) \approx(\bar{x}-x)^{2} \text { and }\left(x^{s} \circ Y_{0}-X_{0}\right)^{\prime}(x) \approx(\bar{x}-x)
$$

for all $x$ (here $\approx$ means that the quotient of the two expressions is in between 3 and 5 ). Now we introduce $(X(x, t), Y(x, t))=f\left(x, y_{0}(x)+t\right)$ for $|t| \leq 2\left(C_{h} b\right)^{n_{k-1} / 2}$. The condition $f\left(x, y_{0}(x)+t\right) \in \Gamma^{m_{k}}(\zeta)$ is expressed by $X(x, t)-x_{\zeta}(Y(x, t))=0$ or, equivalently,

$$
x^{s}(Y(x, t))-X(x, t)=x^{s}(Y(x, t))-x_{\zeta}(Y(x, t))
$$

Observe that if $(x, t)$ satisfies this equation then $|\bar{x}-x| \approx e^{-r_{k-1}}$ and

$$
\left|\partial_{x}\left(x^{s}(Y(x, t))-X(x, t)\right)\right| \approx e^{-r_{k-1}} .
$$

This can be shown as follows. For $t=0$ it follows from (26) and (27):

$$
e^{-2 r_{k-1}} \approx x^{s}\left(Y_{0}(x)\right)-x_{\zeta}\left(Y_{0}(x)\right)=x^{s}\left(Y_{0}(x)\right)-X_{0}(x) \approx(\bar{x}-x)^{2} .
$$

Then it remains true for every $|t| \leq 2\left(C_{h} b\right)^{n_{k-1} / 2}$, because $\left|X(\cdot, t)-X_{0}(\cdot)\right|$ and $\left|Y(\cdot, t)-Y_{0}(\cdot)\right|$, as well as their derivatives, are uniformly bounded by

$$
C \sqrt{b}|t| \leq 2 C \sqrt{b}\left(C_{h} b\right)^{n_{k-1} / 2} \ll e^{-r_{k-1}} .
$$


On the other hand, (26) gives

$$
\left|\partial_{x}\left(x^{s} \circ Y-x_{\zeta} \circ Y\right)\right| \leq C \sqrt{b} e^{-r_{k-1}} \ll e^{-2 r_{k-1}} \approx\left|\partial_{x}\left(x^{s} \circ Y-X\right)\right| .
$$

So, (28) defines $x$ as an implicit function of $t$ in the whole interval $|t| \leq 2\left(C_{h} b\right)^{n_{k-1} / 2}$. Since the $t$-derivatives of all the terms in (28) are bounded by $C \sqrt{b}$, we also get $\left|x^{\prime}(t)\right| \leq C \sqrt{t} e^{r_{k-1}}$.

Now we prove the lemma for large $r_{k-1}$, that is, when $\left(C_{h} b\right)^{n_{k-1}} \geq e^{-4 r_{k-1}}$. We clain that in this case

$$
\operatorname{dist}(\zeta, \eta) \leq C \sqrt{b} e^{-r_{k-1}}<e^{-r_{k-1}} .
$$

Note that this implies both inequalities in the statement. To prove the claim, let $\left(x^{s}(y), y\right)$ and $\left(x_{\zeta}(y), y\right)$ be as before, and $(X(x), Y(x))$ parametrise the unstable side $\gamma^{u}$ that contains $\eta$ Suppose first that $\Gamma^{m_{k}}(\zeta)$ intersects $\gamma^{u}$ at the point $\eta$. Then the length of the segment of $\Gamma^{m_{k}}$ connecting $\zeta$ to $\eta$ is less than $C \sqrt{b} e^{-r_{k-1}}$, as a consequence of the estimates corresponding to (26) and (27), with $X, Y$ in the place of $X_{0}, Y_{0}$. This proves the claim in this case. The other one corresponds to $\Gamma^{m_{k}}$ intersecting a stable side $\gamma^{s}$ at some point $\eta^{\prime}$, with $\eta$ being an endpoint of $\gamma^{s}$. The same argument as before applies both to the segment of $\Gamma^{m_{k}}$ connecting $\zeta$ to $\eta^{\prime}$ and to the stable segment $\gamma^{s}$ : their lengths are shorter than $C \sqrt{b} e^{-r_{k-1}}$. So we get (29) in this case too.

Lemma 3.11. Let $\xi_{k}$ be as in Lemma 3.9. If $r_{j} \leq 5 n_{j}$ for every $1 \leq j \leq k-1$ then

$$
\operatorname{angle}\left(D f^{n_{k}}(z) w_{0}, t\left(f^{n_{k}}\left(\xi_{k}\right)\right)\right) \leq(C b)^{n_{k-1} / 4} .
$$

Proof. During the proof we represent by $C_{a}$ the constant in the statement. In a number of places we assume $C_{a}$ to be large, with respect to a few other constants.

First we treat the case $k=1$. Combining Propositions 2.8.4 and 2.5 we get that $\xi_{1}$ is expanding up to time $n_{1}$. So, recall Proposition 2.3.1, the contracting direction $e_{\xi}=\left(\epsilon_{\xi}, 1\right)$ of order $n_{1}$ at $\xi_{1}$ is well defined and satisfies $\left|\epsilon_{\xi}\right| \leq C \sqrt{b}$. Let $\left(1, \dot{y}_{\xi}\right),\left|\dot{y}_{\xi}\right| \leq 1 / 5$, represent the
tangent direction to $W^{u}(P)$ at $f^{-1}\left(\xi_{1}\right)$. We split

$$
D f\left(f^{-1}\left(\xi_{1}\right)\right)\left(1, \dot{y}_{\xi}\right)=\alpha_{\xi} e_{\xi}+\beta_{\xi} w_{0} .
$$

As in (7), we must have $\left|\alpha_{\xi}\right| \leq C \sqrt{b}$ and $\left|\beta_{\xi}\right| \approx 2 a d_{\mathcal{C}}\left(f^{-1}\left(\xi_{1}\right)\right) \approx 2 a e^{-r_{0}}$. By Proposition 2.3.2, $\left\|D f^{n_{1}}\left(\xi_{1}\right) e_{\xi}\right\| \leq(C b)^{n_{1}}$. Since $t\left(f^{n_{1}}\left(\xi_{1}\right)\right)$ is collinear to $\alpha_{\xi} D f^{n_{1}}\left(\xi_{1}\right) e_{\xi}+\beta_{\xi} D f^{n_{1}}\left(\xi_{1}\right) w_{0}$,

$$
\text { angle }\left(D f^{n_{1}}\left(\xi_{1}\right) w_{0}, t\left(f^{n_{1}}\left(\xi_{1}\right)\right)\right) \leq \frac{\left|\alpha_{\xi}\right|\left\|D f^{n_{1}}\left(\xi_{1}\right) e_{\xi}\right\|}{\left|\beta_{\xi}\right|\left\|D f^{n_{1}}\left(\xi_{1}\right) w_{0}\right\|} \leq C \sqrt{b} e^{r_{0}}(C b)^{n_{1}} \leq(C b)^{n_{1} / 2} .
$$
The last inequality uses $n_{1}=m_{1}>r_{0}$. Moreover, by construction, $\operatorname{dist}\left(f^{j}(\eta), f^{j}(\zeta)\right) \leq(C b)^{j}$
for $0 \leq j \leq n_{1}$. So, using Proposition 2.3.5 we get

$$
\text { angle }\left(D f^{n_{1}}(z) w_{0}, D f^{n_{1}}\left(\xi_{1}\right) w_{0}\right) \leq(C b)^{n_{1} / 2} .
$$

Adding these two angle estimates, we find that angle $\left(D f^{n_{1}}(z) w_{0}, t\left(f^{n_{1}}\left(\xi_{1}\right)\right)\right)$ is bounded by $(C b)^{n_{1} / 2}$. The case $k=1$ of the lemma follows, taking $C_{a}$ larger than this last constant $C$. 
Now we proceed by induction. We use the same notations as in the prof of the previous lemma, in particular, $\zeta=f^{n_{k-1}+1}(z)$ and $\eta=f^{n_{k-1}+1}\left(\xi_{k}\right)$. As before, $\left\|D f^{j}(\eta) w_{0}\right\| \geq 1$ for $1 \leq j \leq m_{k}$. Recall that the distance between iterates of $\zeta$ and $\eta$ is exponentially contracted during the first $m_{k}$ iterates. So, using Lemma 3.10,

$$
\operatorname{dist}\left(f^{j}(\eta), f^{j}(\zeta)\right) \leq(C b)^{n_{k-1} / 2} e^{r_{k-1}}(C b)^{j} \text { for all } 0 \leq j \leq m_{\cdot k} .
$$

By Lipschitz continuity of $D f$, for every $1 \leq j \leq m_{k}$ we have

$$
\left\|D f^{j}(\zeta) w_{0}-D f^{j}(\eta) w_{0}\right\| \leq(C b)^{n_{k-1} / 2} e^{r_{k-1}} .
$$

In view of the assumption $r_{k-1} \leq 5 n_{k-1}$, the last term can be made small by reducing $b$. So, in particular, $\left\|D f^{j}(\zeta) w_{0}\right\| \geq 1 / 2$ for $1 \leq j \leq m_{k}$. Then the contracting directions of order $m_{k}$ $e_{\eta}=\left(\epsilon_{\eta}, 1\right)$ at $\eta$ and $e_{\zeta}=\left(\epsilon_{\zeta}, 1\right)$ at $\zeta$, are well defined and satisfy $\left|\epsilon_{\eta}\right|,\left|\epsilon_{\zeta}\right| \leq C \sqrt{b}$. Let $\left(1, \dot{y}_{\eta}\right)$ and $\left(1, \dot{y}_{\zeta}\right)$, with $\left|\dot{y}_{\eta}\right|,\left|\dot{y}_{\zeta}\right| \leq 1 / 5$, be collinear to $t\left(f^{-1}(\eta)\right)$ and to $D f^{n_{k-1}}(z) w_{0}$, respectively. We split

$$
D f\left(f^{-1}(\eta)\right)\left(1, \dot{y}_{\eta}\right)=\alpha_{\eta} e_{\eta}+\beta_{\eta} u_{0} \quad \text { and } \quad D f\left(f^{-1}(\zeta)\right)\left(1, \dot{y}_{\zeta}\right)=\alpha_{\zeta} e_{\zeta}+\beta_{\zeta} w_{0} .
$$

Then $\left|\alpha_{\eta}\right|$ and $\left|\alpha_{\zeta}\right|$ are bounded by $C \sqrt{b}$, morcover, $\left|\beta_{\eta}\right| \approx 2 a d_{\mathcal{C}}\left(f^{-1}(\eta)\right) \approx 2 a e^{-r_{k-1}}$. The induction assumption means that $\left|\dot{y}_{\eta}-\dot{y}_{\zeta}\right| \leq 2\left(C_{a} b\right)^{n_{k-1} / 4}$. By Lemma 3.10 and $r_{k-1} \leq 5 n_{k-1}$,

$$
\operatorname{dist}\left(f^{-1}(\eta), f^{-1}(\zeta)\right) \leq(C b)^{n_{k-1} / 2} e^{r_{k-1}} \leq(C b)^{n_{k-1} / 4} .
$$

We take $C_{a}$ larger than this last constant $C$. It follows that

$$
\left|\alpha_{\eta}-\alpha_{\zeta}\right| \leq C \sqrt{b}\left(C_{a} b\right)^{n_{k-1} / 4} \text { and }\left|\beta_{\eta}-\beta_{\zeta}\right| \leq C\left(C_{a} b\right)^{n_{k-1} / 4} .
$$

Using the assumption $r_{k-1} \leq 5 n_{k-1}$ once more, we conclude that these expressions are much smaller than $e^{-r_{k-1}}$. In particular, $\left|\beta_{\zeta}\right|$ is also of order $e^{-r_{k-1}}$. Expansivity implies

$$
\left\|D f^{m_{k}}(\eta) e_{\eta}\right\| \leq(C b)^{m_{k}} \quad \text { and }\left\|D f^{n_{k}}(\zeta) e_{\zeta}\right\| \leq(C b)^{m_{k}},
$$

whereas Proposition 2.3.4 and Lemma 3.10 give

$$
\left\|D f^{m_{k}}(\eta) e_{\eta}-D f^{m_{k}}(\zeta) e_{\zeta}\right\| \leq(C b)^{m_{k}} \operatorname{dist}(\eta, \zeta) \leq(C b)^{m_{k}} e^{r_{k-1}}(C b)^{n_{k-1} / 2} .
$$

We take $C_{a} \geq C$ for any of the constants appearing in (31) - (35). Then, combining these estimates through the triangle inequality,

$$
\left\|\frac{\alpha_{\eta} D f^{m_{k}}(\eta) e_{\eta}}{\left|\beta_{\eta}\right|\left\|D f^{m_{k}}(\eta) w_{0}\right\|}-\frac{\alpha_{\zeta} D f^{m_{k}}(\zeta) e_{\zeta}}{\left|\beta_{\zeta}\right|\left\|D f^{m_{k}}\left(\zeta_{k}\right) w_{0}\right\|}\right\| \leq C \sqrt{b}\left(C_{a} b\right)^{n_{k-1} / 4} e^{2 r_{k-1}}\left(C_{a} b\right)^{m_{k}} .
$$

Then, as $t\left(f^{n_{k}}\left(\xi_{k}\right)\right)$ is collinear to $\alpha_{\eta} D f^{m_{k}}(\eta) e_{\eta}+\beta_{\eta} D f^{m_{k}}(\eta) w_{0}$ and $D f^{n_{k}}(z) w_{0}$ is collinear to $\alpha_{\zeta} D f^{m_{k}}(\zeta) e_{\zeta}+\beta_{\zeta} D f^{m_{k}}(\zeta) w_{0}$,

$$
\begin{aligned}
\operatorname{angle}\left(D f^{n_{k}}(z) w_{0},\right. & \left.t\left(f^{n_{k}}\left(\xi_{k}\right)\right)\right) \\
& \leq \operatorname{angle}\left(D f^{m_{k}}(\eta) w_{0}, D f^{m_{k}}(\zeta) w_{0}\right)+C \sqrt{b}\left(C_{a} b\right)^{n_{k-1} / 4} e^{2 r_{k-1}}\left(C_{a} b\right)^{m_{k}} \\
& \leq(C b)^{n_{k-1} / 4} e^{r_{k-1} / 2}(C b)^{m_{k} / 2}+C \sqrt{b}\left(C_{a} b\right)^{n_{k-1} / 4} e^{2 r_{k-1}}\left(C_{a} b\right)^{m_{k}} .
\end{aligned}
$$


For the first inequality compare Lemma 10.1 in [15]. The second inequality follows from P:oposition 2.3.5 combined with (30). Since $n_{k}=n_{k-1}+1+m_{k}$ and $m_{k}>r_{k-1}$, this gives

$$
\text { angle }\left(D f^{n_{k}}(z) w_{0}, t\left(f^{n_{k}}\left(\xi_{k}\right)\right)\right) \leq\left(C_{a} b\right)^{n_{k} / 4} \text {. }
$$

(take $C_{a}$ larger than the other constants in the previous inequality). So, the inductive step is complete. Remark 3.4. More generally, if $0 \leq t \leq k-1$ is such that $r_{j} \leq 5\left(n_{j}-n_{t}\right)$ for all $t<j \leq k-1$
then

$$
\text { angle }\left(D f^{n_{k}-n_{t}-1}\left(f^{n_{t}+1}(z)\right) w_{0}, t\left(f^{n_{k}}\left(\xi_{k}\right)\right)\right) \leq(C b)^{\left(n_{k}-n_{t}-1\right) / 4} .
$$

This is proved in the same way as the lemma, starting the induction at $k=t+1$. Observe that the assumption was used to relate $r_{k-1}$ and $n_{k-1}$, in the context of $(31),(32),(33)$. In the present situation one relates $r_{k-1}$ and $n_{k-1}-n_{t}$ in very much the same way.

Remark 3.5. The hypotheses in Lemma 3.11 and Remark 3.4 are unnecessarily strong: the arguments remain valid if one assumes only $r_{j} \leq|\log C b| n_{j}$ for $1 \leq j \leq k-1$, respectively, $r_{j} \leq|\log C b|\left(n_{j}-n_{t}\right)$ for $t<j \leq k-1$. On the other hand, the statements given above are sufficient for all our purposes in this paper (we never use these more general hypotheses).

Now we start the proof of Lemmas 3.5 and 3.7. Part 1 of either lemma is trivial if $r_{k-1}$ is zero, so we only have to treat the case $r_{k-1} \geq \Delta$. Since $\|D f\| \leq 4$, the definition of $r_{k-1}$ and $p_{k}$ implies $4^{p_{k}+1} e^{-2 \tau_{k-1}} \geq c e^{-\beta\left(p_{k}+1\right)}$. So

$$
m_{k}>p_{k} \geq \frac{2}{\log 4+\beta} r_{k-1}+\log c-1 \geq r_{k-1},
$$

( $\log c-1$ is negligible if $\Delta$ is big enough). This gives Lemma 3.5.1 and Lemma 3.7.1.

Next we prove Lemma 3.5.2. The main point is to check that $p_{1}$ is a good bound period for any point in $L_{i}=f^{-1}\left(\gamma_{i}^{u}\right)$, in the sense of Section 2.2. Suppose first that $\tilde{\zeta}_{0}=\zeta_{0}$, corresponding to cases (a) or (b) of the definition of $i_{0}$. By construction, cf. the last paragraph of Section 3.1,

$$
\text { length }\left(L_{i}\right) \approx \frac{e^{-r_{0}}}{r_{0}^{2}} \leq \frac{e^{-r_{0}}}{\Delta^{2}} \ll e^{-r_{0}} \approx \operatorname{dist}\left(L_{i}, \zeta_{0}\right),
$$

So, (10) is satisfied by these segments $L_{i}$, with a constant $c$ that may be taken as small as wanted by increasing $\Delta=\log (1 / \delta)$. Consequently, for all $j \leq p_{1}+1$ the distances between $f^{j}\left(\zeta_{0}\right)$ and the $j$ th iterates of any two points in $L_{i}$ are comparable up to a factor 2 , or else they are both smaller than $(C b)^{j}$. Using also that

$$
\text { length }\left(f^{j-1}\left(\gamma_{i}^{s}\right)\right) \leq C \sqrt{b}(C b)^{j-1} \ll e^{-\beta j},
$$

for $i=0,1$ and $j \geq 1$, we deduce from the definition of $p_{1}$ that

$$
\operatorname{dist}\left(f^{p_{1}+1}(\xi), f^{p_{1}+1}\left(\zeta_{0}\right)\right) \geq \frac{1}{10} e^{-\beta\left(p_{1}+1\right)} \text { and } \operatorname{dist}\left(f^{j}(\xi), f^{j}\left(\zeta_{0}\right)\right) \leq 10 e^{-\beta j}
$$

for $1 \leq j \leq p_{1}$, for every $\xi \in f^{-1}\left(R\left(i_{0}\right)\right)$. Hence, the conclusions of Proposition 2.8 hold for any point $\xi$ in $L_{0} \cup L_{1}$, with $p_{1}$ in the place of $p(\xi)$. In particular, the slope of $f^{p_{1}}\left(\gamma_{i}^{u}\right)=f^{p_{1}+1}\left(L_{i}\right)$ is less than $(C / \delta) \sqrt{b}<1 / 10$. Now the claim follows from the last statement in Proposition 2.5 . 
Case (e) is treated in the same way, just with $\zeta_{1}$ in the place of $\zeta_{0}$. Cases (d) and (f) are easier, since $p_{1}=0$ and slope $\left(\gamma_{i}^{u}\right)<1 / 10$ comes for free. Finally, case (c) is also similar to (a)(b) in what concerns the unstable segment $\gamma_{1}^{u}$ that is contained in $f\left(\gamma_{1}\right)$, sez Figure 1 . Recall that we defined the binding point to be $\zeta_{1} \in \gamma_{1}$. Using Proposition 2.5 as tefore we conclude that slope $\left(f^{n_{1}}\left(\gamma_{1}^{u}\right)\right)$ is less than $C \sqrt{b}$ at every point. We can not argue in ust the same way for the other unstable segment $\gamma_{0}^{u} \subset f\left(\gamma_{0}\right)$, because of the asymmetry introdiuced by the choice of the binding point. However, this can be easily circumvented using the previous two lemmas as follows. Given $z \in \gamma_{0}^{u}$ take $\xi_{1} \in \gamma_{1}^{u}$ as in Lemma 3.9. Then, by Lemma 3.11,

$$
\text { angle }\left(D f^{n_{1}}(z) w_{0}, t\left(f^{n_{1}}\left(\xi_{1}\right)\right)\right) \leq(C b)^{n_{1} / 4},
$$

and a similar inequality holds with $\xi_{1}$ replaced by $z$. In particular, angle $\left(t\left(f^{n_{1}}(z)\right), t\left(f^{n_{1}}\left(\xi_{1}\right)\right)\right)$ is less than $2(C b)^{n_{1} / 4}$, and then

$$
\text { slope }\left(f^{n_{1}}\left(\gamma_{0}^{u}\right)\right) \leq C \sqrt{b}+2(C b)^{n_{1} / 4} \leq 2 C \sqrt{b} .
$$

The proof of Lemma 3.5.2 is complete. Similar arguments give Lemma 3.7.2.

Part 3 of both Lemmas 3.5 and 3.7 follows immediately from Lemma 3.9: for $z=f^{-n_{k}}\left(z_{0}^{*}\right)$ we may take $\xi_{k}=f^{-n_{k}}\left(z_{1}^{*}\right)$, and then, cf. Remark 3.3,

$$
\operatorname{dist}\left(z_{0}^{*}, z_{1}^{*}\right) \leq(C b)^{n_{k} / 2} \leq \frac{1}{10} e^{-2 \beta n_{k}} \leq \frac{1}{10} d_{\mathcal{C}}\left(z_{i}^{*}\right) .
$$

Finally, part 4 of Lemmas 3.5 and 3.7 can be readily deduced from Lemma 3.11 , in the version given in Remark 3.4. Indeed, let $z_{0}^{*}$ and $z_{1}^{*}$ be the endpoints of $f^{m_{k}}\left(\gamma_{i}^{s}\right)$. We may consider $z=f^{-n_{k}}(\zeta)$ for an arbitrary point $\zeta \in f^{m_{k}}\left(\gamma_{i}^{S}\right)$, and $\xi_{k}=f^{-n_{k}}\left(z_{l}^{*}\right)$ for $l=0$ or $l=1$. The way we have defined itineraries, there exists $0 \leq t \leq k-1$ such that $f^{n_{t}+1}(z)$ and $f^{n_{t}+1}\left(\xi_{k}\right)$ belong to a same long stable leaf $\Gamma_{r_{t}, l_{t}}\left(\tilde{\zeta}_{t}\right)$. By the construction of these leaves, recall Proposition 3.3 and Remark $3.3, r_{j} \leq 2 \beta\left(n_{j}-n_{t}\right)<5\left(n_{j}-n_{t}\right)$ for all $j \geq t+1$. So, we may conclude that

$$
\operatorname{angle}\left(D f^{n_{k}-\left(n_{t}+1\right)}\left(f^{n_{t}+1}(z)\right) w_{0}, t\left(z_{l}^{*}\right)\right)=\operatorname{angle}\left(D f^{n_{k}-\left(n_{t}+1\right)}\left(f^{n_{t}+1}(z)\right) w_{0}, t\left(f^{n_{k}}\left(\xi_{k}\right)\right)\right)
$$

is bounded by $(C b)^{\left(n_{k-1}-n_{t}-1\right) / 4}$. Adding the inequalities corresponding to $l=0$ and $l=1$, we get the claims in Lemmas 3.5.4 and 3.7.4.

$$
\text { angle }\left(t\left(z_{0}^{*}\right), t\left(z_{1}^{*}\right)\right) \leq(C b)^{\left(n_{k-1}-n_{t}-1\right) / 4} \leq \frac{1}{20} e^{-2 \beta\left(n_{k}-n_{t}+1\right)} \leq \frac{1}{10} d_{\mathcal{C}}\left(z_{l}^{*}\right) .
$$

We conclude this section with yet another useful application of these arguments.

Corollary 3.12. Let $0 \leq t<k$ and $z \in \Delta_{0}$ satisfy $r_{i} \leq 5\left(n_{i}-n_{t}\right)$ for every $t<i<k$. Then $f^{n_{t}+1}(z)$ is expanding up to time $n_{k}-n_{t}-1$ : there is $\lambda \geq e^{-20}$ such that

$$
\left\|D f^{j}\left(f^{n_{t}+1}(z)\right) w_{0}\right\| \geq \lambda^{j} \quad \text { for every } 1 \leq j<n_{k}-n_{t} .
$$

Proof. We check that Remark 3.2 can be applied to the point $\zeta=f^{n_{t}}(z)$. Recall that, according to Lemma 3.9 , the height of the rectangle $f^{n_{j}}\left(R\left(i_{0}, \ldots, i_{j}\right)\right)$ does not exceed $(C b)^{n_{j} / 2}$, which is much smaller than $e^{-5 n_{j}} \leq e^{-5\left(n_{j}-n_{t}\right)}$. Therefore, the assumption of the corollary implies that $\operatorname{dist}\left(f^{n_{j}-n_{t}}(\zeta), \tilde{\zeta}_{j}\right) \geq e^{-5\left(n_{j}-n_{t}\right)}$ and $f^{n_{j}-n_{t}}(\zeta)$ is in tangential position relative to $\left(\tilde{\zeta}_{j}, t\left(\tilde{\zeta}_{j}\right)\right)$, for every $j>t$. Using Remarl 3.4 we get more: $\left(f^{n_{j}-n_{t}}(\zeta), D f^{n_{j}-n_{t}-1}(f(\zeta)) w_{0}\right)$ is in tangential position relative to $\left(\tilde{\zeta}_{j}, t\left(\tilde{\zeta}_{j}\right)\right)$, also for every $j>t$. So the assumptions of Remark 3.2 are indeed satisfied, and we may conclude that $f(\zeta)$ is expanding. 


\section{Tangential positions are statistically inevitable}

Now the goal is to show that for Lebesgue almost every point in the basin of attraction, raturns are eventually tangential: some positive iterate satisfies the hypothesis of Corollary 3.12. Given an itinerary $\left(i_{0}, i_{1}, \ldots, i_{k}, \ldots\right)$, with return times $n_{0}<n_{1}<\cdots<n_{k}<\cdots$, we define its close returns $\nu_{0}<\nu_{1}<\cdots<\nu_{s}<\cdots$ as follows: $\nu_{0}=n_{0}=-1$ and, for each $s \geq 0$ we let $\nu_{s+1}=n_{k(s+1)}$ where $k(s+1)$ is maximum such that

$$
r_{j} \leq 5\left(n_{j}-\nu_{s}\right) \text { for all } \nu_{s}<n_{j}<n_{k(s+1)}
$$

The main result in this section is Proposition 4.10: itineraries with many close returns are improbable. Sections 4.1 and 4.2 contain some crucial preparatory results. Throughout, it is understood that all the constants $c, C$ are independent of $k \geq 1$ and $i_{0}, i_{1}, \ldots, i_{k-1}$.

\subsection{Unstable sides are fairly parallel}

The first step is to prove that the tangent directions to the unstable sides of each rectangle $f^{n_{k}}\left(R\left(i_{0}, \ldots, i_{k-1}\right)\right)$ satisfy a Lipschitz condition, expressed in the next proposition.

Proposition 4.1. For any $z_{0}$ and $z_{1}$ in different unstable sides of $f^{n_{k}}\left(R\left(i_{0}, \ldots, i_{k-1}\right)\right)$,

$$
\text { angle }\left(t\left(z_{0}\right), t\left(z_{1}\right)\right) \leq C b^{-1} e^{4\left(n_{k}-\nu_{s}\right)} \operatorname{dist}\left(z_{0}, z_{1}\right)
$$

where $\nu_{s}$ is the last close return before $n_{k}$.

The proof is by induction on $s$ : assuming the conclusion of the proposition at time $\nu_{s}$, we prove that it holds for all $\nu_{s}<n_{k} \leq \nu_{s+1}$. This has two main parts. We begin by obtaining, in Lemma 4.2, an estimate for the angle at time $\nu_{s}+1=n_{k(s)}+1$ in terms of $r_{k(s)}$ only (thus, independent of the history prior to $\nu_{s}$ ). In a second stage, we deduce the proposition for $n_{k}>\nu_{s}$ from this estimate and Lemma 4.4 , which contains the statement that the tangent vectors to the unstable sides of $f^{j}\left(R\left(i_{0}, \ldots, i_{k-1}\right)\right)$ are expanded under $D f^{n_{k}-j}$, for all $\nu_{s}<j<n_{k}$. To keep track of the induction, during the proof we use $C_{p}$ to mean the constant $C$ in the statement
of Proposition 4.1.

Let $x \mapsto\left(x, y_{j}(x)\right), j=0,1$, parametrise the unstable sides of $f^{\nu_{s}}\left(R\left(i_{0}, \ldots, i_{k(s)-1}\right)\right)$, and $y \mapsto\left(x^{s}(y), y\right)$ parametrise the long stable leaf through $f\left(\tilde{\zeta}_{k(s)}\right)$. As before, $\tilde{\zeta}_{k(s)}$ is the binding
critical point associated to the return $\nu_{s}=n_{k(s)}$. We write

$$
\left(\tilde{\xi}_{j}(x), \eta_{j}(x)\right)=f\left(x, y_{j}(x)\right) \quad \text { and } \quad \xi_{j}(x)=\tilde{\xi}_{j}(x)-x^{s}\left(\eta_{j}(x)\right) .
$$

That is, $\xi_{j}(x)$ is the (signed) horizontal distance from the point $\left(\tilde{\xi}_{j}(x), \eta_{j}(x)\right)$ to the stable leaf through $f\left(\tilde{\zeta}_{k(s)}\right)$. Recall that $f(x, y)=\left(1-a x^{2}+R_{1}(x, y), R_{2}(x, y)\right)$ where $R_{1}=R_{1, a}$ and $R_{2}=R_{2, a}$ have $C^{2}$ norm less than $C \sqrt{b}$. So,

$$
\xi_{j}(x)=1-a x^{2}+\psi\left(x, y_{j}(x)\right) \quad \text { with } \quad \psi(x, y)=R_{1}(x, y)-x^{s}\left(R_{2}(x, y)\right)
$$

According to Proposition 2.4 , the first and the second order derivatives of $x^{s}$ are bounded by $C \sqrt{b}$. Therefore, $\psi$ is $C \sqrt{b}$ close to a constant, in the $C^{2}$ norm. Also, cf. Lemma 3.8, the $C^{2}$ 
norms of $y_{0}$ and $y_{1}$ are bounded. These observations imply that $\xi_{j}^{\prime \prime} \approx-2 a$, moreover, the $C^{2}$ norm of $\eta_{j}(x)=R_{2}\left(x, y_{j}(x)\right)$ is less than $C \sqrt{b}$.

In the next lemma we use sl $t(z)$ to represent the slope of tangent vectors in $(\xi, \eta)$ coordinates: sl $t\left(\xi_{j}(x), \eta_{j}(x)\right)=\eta_{j}^{\prime}(x) / \xi_{j}^{\prime}(x)$. Note that we do not take absolute values.

Lemma 4.2. For any $z_{0}$ and $z_{1}$ in the unstable boundary of $f^{\nu_{s}+1}\left(R\left(i_{0}, \ldots, i_{k(s)}\right)\right)$,

$$
\left|\operatorname{si} t\left(z_{0}\right)-\operatorname{sl} t\left(z_{1}\right)\right| \leq C b^{-1} e^{3 r_{k(s)}} \operatorname{dist}\left(z_{0}, z_{1}\right) .
$$

Proof. For the time being we suppose that $z_{0}$ and $z_{1}$ are in different unstable sides of the rectangle $f^{\nu_{s}+1}\left(R\left(i_{0}, \ldots, i_{k(s)}\right)\right)$. There are two cases to be considered, cf. Figure 4 .

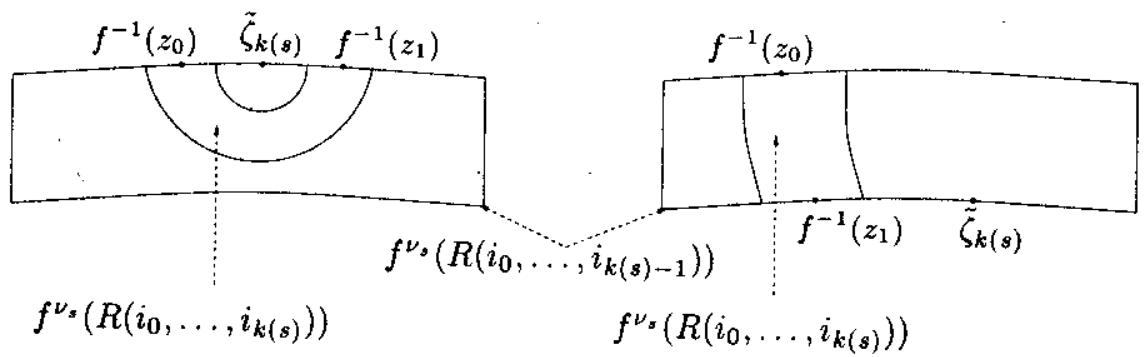

Figure 4:

First, we suppose that $f^{-1}\left(z_{0}\right)$ and $f^{-1}\left(z_{1}\right)$ belong to a same unstable side of the rectangle $f^{\nu \cdot}\left(R\left(i_{0}, \ldots, i_{k(s)-1}\right)\right)$, cf. cases (a), (b), (a1), (b1) in Section 3.2. This corresponds to the left hand half of Figure 4. Up to interchanging the roles of $y_{0}$ and $y_{1}$, we may suppose that this is the unstable side parametrised by $\left(x, y_{0}(x)\right)$. Then $\tilde{\zeta}_{k(s)}=\left(x_{c}, y_{0}\left(x_{c}\right)\right)$ for some $x_{c}$. Recall that $\left|\xi_{0}(x)\right|$ is the horizontal distance from $\left(\tilde{\xi}_{0}(x), \eta_{0}(x)\right)$ to the long stable leaf through $\tilde{\zeta}_{k(s)}$. The fact that $\tilde{\zeta}_{k(s)}$ is a critical point translates into $\xi_{0}^{\prime}\left(x_{c}\right)=0$ and $\xi_{0}\left(x_{c}\right)=0$. Then, since $\xi_{0}^{\prime \prime} \approx-2 a$,

$$
\xi_{0}^{\prime}(x) \approx-2 a\left(x-x_{c}\right) \text { and } \xi_{0}(x) \approx-a\left(x-x_{c}\right)^{2}
$$

for every $x$. Let us write $z_{0}=f\left(x_{0}, y_{0}\left(x_{0}\right)\right)$ and $z_{1}=f\left(x_{1}, y_{0}\left(x_{1}\right)\right)$, for some $x_{0}$ and $x_{1}$. Then $e^{-2 r_{k}} \approx\left|\xi_{0}\left(x_{j}\right)\right| \approx a\left(x_{j}-x_{c}\right)^{2}$, and so $\left|\xi_{0}^{\prime}\left(x_{j}\right)\right| \approx 2 a\left|x_{j}-x_{c}\right| \geq c e^{-r_{k(s)}}$, for $j=0$, 1. In the case we are dealing with $f^{-1}\left(z_{0}\right)$ and $f^{-1}\left(z_{0}\right)$ are to opposite sides of the critical point $\tilde{\zeta}_{k(s)}$. So, $\left|x_{0}-x_{1}\right| \approx\left|x_{0}-x_{c}\right|+\left|x_{1}-x_{c}\right| \geq c e^{-r_{k(s)}}$. Since $\left\|D f^{-1}\right\| \leq 4 J / b$, it follows that

$$
\operatorname{dist}\left(z_{0}, z_{1}\right) \geq c b\left(\left|x_{0}-x_{1}\right|+\left|y_{0}\left(x_{0}\right)-y_{1}\left(x_{1}\right)\right|\right) \geq c b e^{-r_{k(s)}} .
$$

This gives

$$
\left|\operatorname{sl} t\left(z_{0}\right)-\operatorname{sl} t\left(z_{1}\right)\right| \leq \frac{\left|\eta_{0}^{\prime}\left(x_{0}\right)\right|}{\left|\xi_{0}^{\prime}\left(x_{0}\right)\right|}+\frac{\left|\eta_{0}^{\prime}\left(x_{1}\right)\right|}{\left|\xi_{0}^{\prime}\left(x_{1}\right)\right|} \leq C \sqrt{b} e^{r_{k(s)}} \leq C b^{-1 / 2} e^{2 r_{b(s)}} \operatorname{dist}\left(z_{0}, z_{1}\right),
$$

which is even stronger than the claim in the lemma.

Now we treat the situation when $f^{-1}\left(z_{0}\right)$ and $f^{-1}\left(z_{1}\right)$ are in different unstable sides of the rectangle $f^{\nu_{*}}\left(R\left(i_{0}, \ldots, i_{k(s)-1}\right)\right)$, cf. (c), (d), (e), (c1) in Section 3.2. See the right hand half of 
Figure 4. Again, interchanging $y_{0}$ and $y_{1}$ if necessary, we may suppose that the binding (ritical point is $\left(x_{c}, y_{1}\left(x_{c}\right)\right)$ for some $x_{c}$. We write

$$
\xi_{0}(x)=\xi_{1}(x)+\rho\left(x,\left(y_{0}-y_{1}\right)(x)\right), \quad \text { where } \rho(x, h)=\psi\left(x, y_{1}(x)+h\right)-\psi\left(x, y_{1}(x)\right) .
$$

Recall that the function $\psi$, which was introduced in (39), is $C \sqrt{b}$ close to a constant, in the $C^{2}$ topology. As a consequence, the $C^{2}$ norm of $\rho$ is less than $C \sqrt{b}$. Let $x_{0}, x_{1}$ be defiried by $z_{0}=f\left(x_{0}, y_{0}\left(x_{0}\right)\right)$ and $z_{1}=f\left(x_{1}, y_{1}\left(x_{1}\right)\right)$. We have

$$
\begin{aligned}
\xi_{0}^{\prime}\left(x_{0}\right)-\xi_{1}^{\prime}\left(x_{1}\right)=\left(\xi_{1}^{\prime}\left(x_{0}\right)-\xi_{1}^{\prime}\left(x_{1}\right)\right) & +\partial_{x} \rho\left(x_{0},\left(y_{0}-y_{1}\right)\left(x_{0}\right)\right) \\
& +\partial_{y} \rho\left(x_{0},\left(y_{0}-y_{1}\right)\left(x_{0}\right)\right)\left(y_{0}-y_{1}\right)^{\prime}\left(x_{0}\right) .
\end{aligned}
$$

The first term on the right is bounded by $4\left|x_{0}-x_{1}\right|$. Since $\rho(x, 0)=\partial_{x} \rho(x, 0)=0$ for all $x$, the second one is bounded by $C \sqrt{b}\left|\left(y_{0}-y_{1}\right)\left(x_{0}\right)\right|$. As for the last term, we write it as

$$
\left[\partial_{y} \rho\left(x_{0},\left(y_{0}-y_{1}\right)\left(x_{0}\right)\right)-\partial_{y} \rho\left(x_{0}, y_{*}\right)\right]\left(y_{0}-y_{1}\right)^{\prime}\left(x_{0}\right)+\partial_{y} \rho\left(x_{0}, y_{*}\right)\left(y_{0}-y_{1}\right)^{\prime}\left(x_{0}\right)
$$
where $y_{*}$ is any point in the interval bounded by 0 and $\left(y_{0}-y_{1}\right)(x)$, having the mean value
property:

$$
\partial_{y} \rho\left(x_{0}, y_{*}\right)\left(y_{0}-y_{1}\right)\left(x_{0}\right)=\rho\left(x_{0},\left(y_{0}-y_{1}\right)\left(x_{0}\right)\right) .
$$

Since $\left|\left(y_{0}-y_{1}\right)^{\prime}\right| \leq 1$, the first term in (40) is less than

$$
C \sqrt{b}\left|\left(y_{0}-y_{1}\right)\left(x_{0}\right)-y_{*}\right| \leq C \sqrt{b}\left|\left(y_{0}-y_{1}\right)\left(x_{0}\right)\right| .
$$
Now we use induction: assuming that Proposition 4.1 holds for the points $\left(x_{0}, y_{0}\left(x_{0}\right)\right)$ and
$\left(x_{0}, y_{1}\left(x_{0}\right)\right)$ at time $n_{k}=\nu_{s}$ ensures that

$$
\left|\left(y_{1}-y_{0}\right)^{\prime}\left(x_{0}\right)\right| \leq L_{s}\left|\left(y_{1}-y_{0}\right)\left(x_{0}\right)\right|, \quad L_{s}=C_{p} b^{-1} e^{4\left(\nu_{s}-\nu_{s-1}\right)} .
$$

Therefore, using (41),

$$
\left|\partial_{y} \rho\left(x_{0}, y_{*}\right)\left(y_{0}-y_{1}\right)^{\prime}\left(x_{0}\right)\right| \leq L_{s}\left|\rho\left(x_{0},\left(y_{0}-y_{1}\right)\left(x_{0}\right)\right)\right|=L_{s}\left|\left(\xi_{0}-\xi_{1}\right)\left(x_{0}\right)\right| .
$$

At this point, putting the previous estimates together, we have shown that

$$
\left|\xi_{0}^{\prime}\left(x_{0}\right)-\xi_{1}^{\prime}\left(x_{1}\right)\right| \leq C\left|x_{0}-x_{1}\right|+C \sqrt{b}\left|\left(y_{0}-y_{1}\right)\left(x_{0}\right)\right|+L_{s}\left|\left(\xi_{0}-\xi_{1}\right)\left(x_{0}\right)\right| .
$$

Moreover, $\left|\left(y_{0}-y_{1}\right)\left(x_{0}\right)\right| \leq\left|y_{0}\left(x_{0}\right)-y_{1}\left(x_{1}\right)\right|+C\left|x_{0}-x_{1}\right|$, and similarly for $\left(\xi_{0}-\xi_{1}\right)$, since the derivatives of $y_{1}$ and $\xi_{1}$ are uniformly bounded. Hence, the previous inequality implies

$$
\left|\xi_{0}^{\prime}\left(x_{0}\right)-\xi_{1}^{\prime}\left(x_{1}\right)\right| \leq\left(C+L_{s}\right)\left|x_{0}-x_{1}\right|+C \sqrt{b}\left|y_{0}\left(x_{0}\right)-y_{1}\left(x_{1}\right)\right|+L_{s}\left|\xi_{0}\left(x_{0}\right)-\xi_{1}\left(x_{1}\right)\right| .
$$

Moreover, we may replace $C+L_{s}$ by $C L_{s}$ (possibly with a larger $C$ ), since $L_{s} \geq 1$.

In a similar fashion, we write $\eta_{0}(x)=\eta_{1}(x)+\theta\left(x,\left(y_{0}-y_{1}\right)(x)\right)$, where

$$
\theta(x, h)=R_{2}\left(x, y_{1}(x)+h\right)-R_{2}\left(x, y_{1}(x)\right)
$$


has $C^{2}$ norm less than $C \sqrt{b}$, and satisfies $\theta(x, 0)=\partial_{x} \theta(x, 0)=0$ for every $\dot{x}$. Then we conclude, as before, that

$$
\left|\eta_{0}^{\prime}\left(x_{0}\right)-\eta_{1}^{\prime}\left(x_{\mathfrak{1}}\right)\right| \leq C L_{s} \sqrt{b}\left|x_{0}-x_{1}\right|+C \sqrt{b}\left|y_{0}\left(x_{0}\right)-y_{1}\left(x_{1}\right)\right|+L_{s}\left|\eta_{0}\left(x_{0}\right)-\eta_{1}\left(x_{1}\right)\right| .
$$

Observe that the first term comes with a better factor $C \sqrt{b}$ than the corresponding one in (42). This is because $\left|\eta_{j}^{\prime}\left(x_{0}\right)-\eta_{j}^{\prime}\left(x_{1}\right)\right| \leq C \sqrt{b}\left|x_{0}-x_{1}\right|$ and $\left|\eta_{j}\left(x_{0}\right)-\eta_{j}\left(x_{1}\right)\right| \leq C \sqrt{b}\left|x_{0}-x_{1}\right|$, which are better than the corresponding estimates for $\xi_{j}^{\prime}$ and $\xi_{j}$. Putting (42) and (43) together with $\left|\xi_{j}^{\prime}\right| \leq C$ and $\left|\eta_{j}^{\prime}\right| \leq C \sqrt{b}$, we find that $\left|\eta_{0}^{\prime}\left(x_{0}\right)\right|\left|\xi_{1}^{\prime}\left(x_{1}\right)-\xi_{0}^{\prime}\left(x_{0}\right)\right|+\left|\xi_{0}^{\prime}\left(x_{0}\right)\right|\left|\eta_{0}^{\prime}\left(x_{0}\right)-\eta_{1}^{\prime}\left(x_{1}\right)\right|$ is bounded by

$$
C\left(\sqrt{b}\left|y_{0}\left(x_{0}\right)-y_{1}\left(x_{1}\right)\right|+\sqrt{b} L_{s}\left|x_{0}-x_{1}\right|+L_{s}\left|\xi_{0}\left(x_{0}\right)-\xi_{1}\left(x_{1}\right)\right|+L_{s}\left|\eta_{0}\left(x_{0}\right)-\eta_{1}\left(x_{1}\right)\right|\right) .
$$

It is clear that the two last terms are bounded by $C L_{s} \operatorname{dist}\left(z_{0}, z_{1}\right)$. We also want to bound the first two terms by some multiple of $\operatorname{dist}\left(z_{0}, z_{1}\right)$. For this we apply the mean value term to $f^{-1}$ : $|\operatorname{det} D f| \geq c b$ gives $\left\|D f^{-1}\right\| \leq C b^{-1}$, and so

$$
\left|y_{0}\left(x_{0}\right)-y_{1}\left(x_{1}\right)\right| \leq C b^{-1} \operatorname{dist}\left(z_{0}, z_{1}\right) .
$$

There is a similar estimate for $\left|x_{0}-x_{1}\right|$ but, in fact, we can do slightly better: since $\left|\partial_{y} R_{1}\right|,\left|\partial_{y} R_{2}\right|$ are less than $C \sqrt{b}$, the derivative of the first component of $D f^{-1}$ is bounded by $C b^{-1 / 2}$, and so the mean value theorem gives

$$
\left|x_{0}-x_{1}\right| \leq C b^{-1 / 2} \operatorname{dist}\left(z_{0}, z_{1}\right) .
$$

Replacing these remarks in (44) we obtain

$$
\left|\eta_{0}^{\prime}\left(x_{0}\right) \xi_{1}^{\prime}\left(x_{1}\right)-\xi_{0}^{\prime}\left(x_{0}\right) \eta_{1}^{\prime}\left(x_{1}\right)\right| \leq C b^{-1 / 2} \operatorname{dist}\left(z_{0}, z_{1}\right)+C L_{s} \operatorname{dist}\left(z_{0}, z_{1}\right) .
$$

A key remark is that,

$$
L_{s} \leq C_{p} b^{-1} e^{-\left(\nu_{s}-\nu_{s-1}\right)} e^{r_{k(s)}} \leq b^{-1} e^{r_{k(s)}}
$$

because $r_{k(s)}>5\left(\nu_{s}-\nu_{s-1}\right)$ (since $\nu_{s}$ is a close return), and $\nu_{s}-\nu_{s-1} \geq p_{k(s-1)}$ can be made arbitrarily large by taking $\delta$ sufficiently small. Thus, (46) gives

$$
\left|\eta_{0}^{\prime}\left(x_{0}\right) \xi_{1}^{\prime}\left(x_{1}\right)-\xi_{0}^{\prime}\left(x_{0}\right) \eta_{1}^{\prime}\left(x_{1}\right)\right| \leq C b^{-1} e^{r_{k(s)}} \operatorname{dist}\left(z_{0}, z_{1}\right),
$$

where the constant $C$ does not depend on $C_{p}$. Furthermore,

$$
\left|\xi_{1}^{\prime}\left(x_{1}\right)\right| \geq c\left|x_{1}-x_{c}\right| \geq c e^{-r_{k(s)}} \quad \text { and } \quad\left|\xi_{0}^{\prime}\left(x_{0}\right)\right| \geq c e^{-r_{k(s)}} .
$$

The first claim follows easily from $\xi_{1}^{\prime}\left(x_{c}\right)=0$ and $\xi_{1}^{\prime \prime} \approx-2 a$. The second one is slightly trickier, because may not be zero $\xi_{0}^{\prime}\left(x_{c}\right)=0$ : the critical $\tilde{\zeta}_{k(s)}=\left(x_{c}, y_{1}\left(x_{c}\right)\right)$ is in the unstable side parametrised by $\left(x, y_{1}(x)\right)$ not $\left(x, y_{0}(x)\right)$. To overcome this, we let $\bar{x}_{c}$ be the unique solution of $\xi_{0}^{\prime}\left(\bar{x}_{c}\right)=0$. Then $\left|\xi_{0}^{\prime}\left(x_{0}\right)\right| \approx 2 a\left|x_{0}-\bar{x}_{c}\right|$, and

$$
a\left|x_{0}-\bar{x}_{c}\right|^{2} \approx\left|\xi_{0}\left(\bar{x}_{c}\right)-\xi_{0}\left(x_{0}\right)\right| \geq\left|\xi_{1}\left(x_{c}\right)-\xi_{0}\left(x_{0}\right)\right| \geq e^{-2 r_{k(*)}},
$$


recall Proposition 3.3. This completes the proof of (48). Then, combining (47) with (48),

$$
\left|\operatorname{sl} t\left(z_{0}\right)-\operatorname{sl} t\left(z_{1}\right)\right|=\left|\frac{\eta_{0}^{\prime}\left(x_{0}\right)}{\xi_{0}^{\prime}\left(x_{0}\right)}-\frac{\eta_{1}^{\prime}\left(x_{1}\right)}{\xi_{1}^{\prime}\left(x_{1}\right)}\right| \leq \frac{C b^{-1} e^{r_{k(o)}} \operatorname{dist}\left(z_{0}, z_{1}\right)}{\left|\xi_{0}^{\prime}\left(x_{0}\right)\right|\left|\xi_{1}^{\prime}\left(x_{1}\right)\right|} \leq C b^{-1} e^{3 r_{k(s)}} \operatorname{dist}\left(z_{0}, z_{1}\right) .
$$

This proves the lemma in this case.

All that is left is the case when $z_{0}$ and $z_{1}$ are in a same unstable side of $f^{\nu_{*}+1}\left(R\left(i_{0}, \ldots, i_{k(s)}\right)\right)$. Equivalently, $f^{-1}\left(z_{0}\right)$ and $f^{-1}\left(z_{1}\right)$ are in a same unstable side of $f^{\nu_{0}}\left(R\left(i_{0}, \ldots, i_{k(s)}\right)\right)$, see Figure 4. Then there are $x_{0}, x_{1}$ so that $z_{0}=f\left(x_{0}, y_{j}\left(x_{0}\right)\right)$ and $z_{1}=f\left(x_{1}, y_{j}\left(x_{1}\right)\right)$, for either $j=0$ or $j=1$. The binding critical point $\tilde{\zeta}_{k(s)}=\left(x_{c}, y_{i}\left(x_{c}\right)\right)$. Here $i$ may differ from $j$ but, for the same reasons as in the previous paragraph, we always have $\left|\xi_{j}^{\prime}\left(x_{0}\right)\right| \geq c e^{-r_{k(s)}}$ and $\left|\xi_{j}^{\prime}\left(x_{1}\right)\right| \geq c e^{-r_{k(s)}}$.
So,

$$
\left|\operatorname{si} t\left(z_{0}\right)-\operatorname{sl} t\left(z_{1}\right)\right|=\left|\frac{\eta_{j}^{\prime}\left(x_{0}\right)}{\xi_{j}^{\prime}\left(x_{0}\right)}-\frac{\eta_{j}^{\prime}\left(x_{1}\right)}{\xi_{j}^{\prime}\left(x_{1}\right)}\right| \leq C \sqrt{b} e^{2 r_{k(s)}}\left|x_{0}-x_{1}\right| \leq C e^{2 r_{k(s)}} \operatorname{dist}\left(z_{0}, z_{1}\right),
$$
where the last inequality uses (45). This is a stronger fact than we claimed. The proof of
Lemma 4.2 is now complete.

Recall that we consider $\nu_{s}=n_{k(s)}$ to be the last close return before $n_{k}$. By Corollary 3.12, every $z \in f^{\nu_{s}+1}\left(R\left(i_{0}, \ldots, i_{k}\right)\right)$ is expanding up to time $\mu=n_{k}-\nu_{s}-1$. Let $e_{\mu}(z)$ denote the contractive direction of order $\mu$ at $z$. Just as in the previous lemma, the constants $C$ in the
next corollary do not depend on $C_{p}$, and this is important for what follows.

Corollary 4.3. There is a $C^{1}$ vector field $v_{0}=(1,0)+\phi e_{\mu}$ defined on $f^{\nu_{s}+1}\left(R\left(i_{0}, \ldots, i_{k}\right)\right)$ and tangent to the unstable sides of $i$, with $|\phi| \leq C \sqrt{b} e^{r_{k(s)}}$ and $\|D \phi\| \leq C b^{-1} e^{\left.3 r_{b(s)}\right)}$.

Proof. First we define $\phi(z)$ for the points $z=\left(\xi_{j}(x), \eta_{j}(x)\right)$ in each of the unstable sides of $f^{\nu+1}\left(R\left(i_{0}, \ldots, i_{k}\right)\right)$, by the condition that $(1,0)+\phi(z) e_{\mu}(z)$ be collinear to the tangent
direction $t(z)$. Writing

$$
t(z)=\xi_{j}^{\prime}(x) \partial_{\xi}+\eta_{j}^{\prime}(x) \partial_{\eta} \quad \text { and } \quad e_{\mu}(z)=(\epsilon(z), 1)=\left[\epsilon(z)-\left(x^{s}\right)^{\prime}\left(\eta_{j}(x)\right)\right] \partial_{\xi}+\partial_{\eta},
$$

this means that

$$
\phi(z)=\frac{\eta_{j}^{\prime}(x) / \xi_{j}^{\prime}(x)}{1-\left[\epsilon(z)-\left(x^{s}\right)^{\prime}\left(\eta_{j}(x)\right)\right]\left[\eta_{j}^{\prime}(x) / \xi_{j}^{\prime}(x)\right]} .
$$
Denote $\tilde{\zeta}_{k(s)}=\left(\xi_{c}, \eta_{c}\right)$ the binding critical point. Proposition 2.3 .2 and Proposition 2.4 imply
$\left|D\left(\epsilon-\left(x^{s}\right)^{\prime}\right)\right| \leq C \sqrt{b}$, and so

$$
\left|\left[\epsilon\left(\tilde{\zeta}_{k(s)}\right)-\left(x^{s}\right)^{\prime}\left(\eta_{c}\right)\right]-\left[\epsilon(z)-\left(x^{s}\right)^{\prime}\left(\eta_{j}(x)\right)\right]\right| \leq C \sqrt{b} \operatorname{dist}\left(z, \tilde{\zeta}_{k(s)}\right) \leq C \sqrt{b} e^{-r_{k(s)}} .
$$

By Proposition 2.3.2 and Lemma 3.7.1, $\left|\epsilon\left(\tilde{\zeta}_{k(s)}\right)-\left(x^{s}\right)^{\prime}\left(\eta_{c}\right)\right| \leq(C b)^{\mu} \ll C \sqrt{b} e^{-r_{k(s)}}$. So,

$$
\left|\epsilon(z)-\left(x^{s}\right)^{\prime}\left(\eta_{j}(x)\right)\right|\left|\eta_{j}^{\prime}(x) / \xi_{j}^{\prime}(x)\right| \leq C \sqrt{b} e^{-r_{k(s)}} C \sqrt{b} e^{r_{k(s)}} \ll 1 .
$$

It immediately follows that $|\phi(z)| \leq 2|\operatorname{sl} t(z)| \leq C \sqrt{b} e^{r_{k(0)}}$. It is clear that $\phi$ is $C^{1}$ on each unstable side. Moreover, its derivative is bounded by $C b^{-1} e^{3 r_{k(s)}}$, as a consequence of the 
Lipschitz estimate for the slopes $\eta_{j}^{\prime}(x) / \xi_{j}^{\prime}(x)$ provided by Lemma 4.2. This lemma also implies that $\phi$ is Lipschitz continuous on the union of the two unstable sides, with Lipschitz constant $C b^{-1} e^{3 r_{k(\bullet)}}$. Therefore, it can be $C^{1}$ extended to the whole rectangle $f^{\nu_{0}+1}\left(R\left(i_{0}, \ldots, i_{k}\right)\right)$, preserving the bounds on the function and the derivative. We still denote this extension by $\phi$, then we define $v_{0}=(1,0)+\phi e_{\mu}$ on the whole rectangle.

Next, we introduce the projectivization $f_{*}$ of $D f$, given by $f_{*}(z, v)=D f(z) v /\|D f(z) v\|$, and define vector fields $v_{j}$ on $f^{\nu_{*}+j+1}\left(R\left(i_{0}, \ldots, i_{k}\right)\right)$, for $1 \leq j \leq \mu$, by push-forward under $f_{*}$ :

$$
v_{j}(\xi)=f_{*}\left(f^{-1}(\xi), v_{j-1}\left(f^{-1}(\xi)\right)\right) .
$$

Of course, each $v_{j}$ is tangent to the unstable sides of $f^{\nu_{s}+j+1}\left(R\left(i_{0}, \ldots, i_{k-1}\right)\right)$.

Lemma 4.4. Given $\zeta \in f^{n_{k}}\left(R\left(i_{0}, \ldots, i_{k-1}\right)\right)$, let $\zeta_{i}=f^{-\mu+i}(\zeta)$ for each $0 \leq i \leq \mu$. Then,

$$
\left\|D f^{i}\left(\zeta_{\mu-i}\right) v_{\mu-i}\left(\zeta_{\mu-i}\right)\right\| \geq 1 \text { for all } 0 \leq i \leq \mu .
$$

Proof. Note that, given any return $\nu_{s}<n_{j}<n_{k}$, the angle between $v_{n_{j}-\nu_{s}-1}\left(f^{n_{j}-\nu_{s}-1}\left(\zeta_{0}\right)\right)$ and $D f^{n_{j}-\nu_{s}-1}\left(\zeta_{0}\right) w_{0}$ is much smaller than $\operatorname{dist}\left(f^{n_{j}-\nu_{s}-1}\left(\zeta_{0}\right), \tilde{\zeta}_{j}\right)$. This is because $v_{0}=w_{0}+\phi e_{\mu}$, where $w_{0}$ is expanded (Propositions 2.5 and 2.8), and $e_{\mu}$ is contracted by positive iterates (Proposition 2.3). By Remark 3.4, with $t=k(s)$, the same is true for the angle between $D f^{n_{j}-\nu_{s}-1}\left(\zeta_{0}\right) w_{0}$ and $t\left(f^{n_{j}}\left(\xi_{j}\right)\right)$. In view of Proposition 2.7, these two facts ensure that $\left(f^{n_{j}-\nu_{0}-1}\left(\zeta_{0}\right), v_{n_{j}-\nu_{s}-1}\left(f^{n_{j}-\nu_{s}-1}\left(\zeta_{0}\right)\right)\right.$ is in tangential position relative to $\left(\tilde{\zeta}_{j}, t\left(\tilde{\zeta}_{j}\right)\right)$, for every return $n_{j}$ in between $\nu_{s}$ and $n_{k}$. Now the proof is similar to that of [2, Lemma 7.13].

Proposition 4.1 is an immediate consequence of the next lemma (with the same constants).

Lemma 4.5. $\left\|D v_{\mu}(\zeta)\right\| \leq C b^{-1} e^{4\left(n_{k}-\nu_{s}\right)} \quad$ at every $\zeta \in f^{n_{k}}\left(R\left(i_{0}, \ldots, i_{k-1}\right)\right)$.

Proof. We list a few elementary facts that are used in the proof. Let $\xi$ be a generic point and $v$ be any norm 1 tangent vector at $\xi$. Then $\partial_{v} f_{*}(\xi, v) \dot{v}$ coincides with the component of $D f(\xi)(\dot{v}) /\|D f(\xi) v\|$ orthogonal to $D f(\xi) v$, for any vector $\dot{v}$ tangent to the $v$-direction at $(\xi, v)$. In particular,

$$
\left|\partial_{v} f_{*}(\xi, v)\right||| D f(\xi) v \|^{2}=|\operatorname{det} D f(\xi)| .
$$

Similarly, $\partial_{\xi} f_{*}(\xi, v) \dot{\xi}$ coincides with the component of $D^{2} f(\xi)(\dot{\xi}, v) /\|D f(\xi) v\|$ orthogonal to $D f(x) v$, for any tangent vector $\dot{\xi}$ tangent to the $\xi$-direction at $(\xi, v)$. As a consequence, the norm of $\partial_{\xi} f_{*}(\xi, v) D f(\xi)^{-1} \dot{\eta}$ is bounded by

$$
\left\|D^{2} f(\xi)\left(D f(\xi)^{-1} \dot{\eta}, \frac{v}{\|D f(\xi) v\|}\right)\right\| \leq \frac{\left\|D^{2} f(\xi)\right\|}{|\operatorname{det} D f(\xi)|}\|\dot{\eta}\|
$$

for any tangent vector $\dot{\eta}$ tangent to the $\xi$-direction at $(f(\xi), D f(\xi) v)$.

Taking derivatives in the definition of $v_{\mu}$ yields

$$
\begin{array}{r}
D v_{\mu}(\zeta)=\sum_{j=0}^{\mu-1} \partial_{v} f_{*}^{j}\left(\zeta_{\mu-j}, v_{\mu-j}\right) \partial_{\xi} f_{*}\left(\zeta_{\mu-j-1}, v_{\mu-j-1}\right) D f^{-j-1}(\zeta) \\
+\partial_{v} f_{*}^{\mu}\left(\zeta_{0}, v_{0}\right) D v_{0}\left(\zeta_{0}\right) D f^{-\mu}(\zeta)
\end{array}
$$


where $v_{\mu-j}$ means $v_{\mu-j}\left(\zeta_{\mu-j}\right)$. For every $j \geq 1$,

$$
\left\|\partial_{v} f_{*}^{j}\left(\zeta_{\mu-j}, v_{\mu-j}\right)\right\|=\prod_{i=\mu-j}^{\mu-1} \frac{\left|\operatorname{det} D f\left(\zeta_{i}\right)\right|}{\left\|D f\left(\zeta_{i}\right) v_{i}\right\|^{2}}=\frac{\left|\operatorname{det} D f^{j}\left(\zeta_{\mu-j}\right)\right|}{\left\|D f^{j}\left(\zeta_{\mu-j}\right) v_{\mu-j}\right\|^{2}} .
$$

On the other hand,

Moreover,

$$
\left\|D f^{-j}(\zeta)\right\| \leq \frac{\left\|D f^{j}\left(\zeta_{\mu-j}\right)\right\|}{\left|\operatorname{det} D f^{j}\left(\zeta_{\mu-j}\right)\right|}
$$

Replacing all this in (50),

$$
\left\|\partial_{\xi} f_{*}\left(\zeta_{\mu-j-1}, v_{\mu-j-1}\right) D f^{-1}\left(\zeta_{\mu-j}\right)\right\| \leq \frac{\left\|D^{2} f\left(\zeta_{\mu-j}\right)\right\|}{\left|\operatorname{det} D f\left(\zeta_{\mu-j}\right)\right|} \leq C b^{-1}
$$

$$
\left\|D v_{\mu}(\zeta)\right\| \leq \sum_{j=0}^{\mu-1} C b^{-1} \frac{\left\|D f^{j}\left(\zeta_{\mu-j}\right)\right\|}{\left\|D f^{j}\left(\zeta_{\mu-j}\right) v_{\mu-j}\right\|^{2}}+\frac{\left\|D f^{\mu}\left(\zeta_{0}\right)\right\|}{\left\|D f^{\mu}\left(\zeta_{0}\right) v_{0}\right\|^{2}}\left\|D v_{0}\left(\zeta_{0}\right)\right\| .
$$

So, in view of Corollary 4.3 and Lemma 4.4,

$$
\left\|D v_{\mu}(\zeta)\right\| \leq \sum_{j=0}^{\mu-1} C b^{-1} 4^{j}+4^{\mu} C b^{-1} e^{3 r_{k(s)}} \leq C_{p} b^{-1} e^{4 \mu},
$$

as long as we choose $C_{p}$ sufficiently large with respect to the other constants. This can be done since the constants $C$ in Lemma 4.2 and Corollary 4.3 were taken independent of $C_{p}$, recall (47). The last inequality also uses $\mu>p_{k(s)} \geq(4 / 3) r_{k(s)}$, which is a consequence of $(36)$.

\subsection{Area distortion bounds}

Next, we obtain a uniform bound for the distortion of the Jacobian on trajectories sharing a same itinerary:

Proposition 4.6. Given any $k \geq 1$ and $i_{0}, \ldots, i_{k-1}$,

$$
\frac{\left|\operatorname{det} D f^{n-l}\left(f^{l}(z)\right)\right|}{\left|\operatorname{det} D f^{n-l}\left(f^{l}(w)\right)\right|} \leq C
$$

for every $z, w \in R\left(i_{0}, \ldots, i_{k-1}\right)$ and every $0 \leq l<n \leq n_{k}$.

Let us observe that this statement is trivial when the map $f$ has constant Jacobian, e.g. the Hénon model. In the general case, the proof is based on the following two lemmas.

Lemma 4.7. Given any $k \geq 1$ and $i_{0}, \ldots, i_{k-1}$, we have

$$
\sum_{j=0}^{n_{k}-1} \text { length }\left(f^{j}\left(\gamma^{u}\right)\right) \leq C
$$

for either of the unstable sides $\gamma^{u}$ of $R\left(i_{0}, \ldots, i_{k-1}\right)$. 
Proof. The statement follows from ideas from [2, Lemma 7.8]. We only outline the main points, referring the reader to [2] for details. Let $\ell_{i}=\operatorname{length}\left(f^{n_{i}}\left(\gamma^{u}\right)\right)$, for $0 \leq i \leq k$.

The first step is to show that the sum over any free period $\left[n_{i-1}+p_{i}+1, \imath_{i}-1\right]$ is bounded by $C \ell_{i}$.

$$
\sum_{j \in n_{i-1}+p_{i}+1}^{n_{i}-1} \operatorname{length}\left(f^{j}\left(\gamma^{u}\right)\right) \leq C \ell_{i} .
$$

To prove this, one notes that Proposition 2.5 implies that the lengths grow exponentially fast during free periods: length $\left(f^{j}\left(\gamma^{u}\right)\right) \leq C \sigma_{0}^{j-n_{i}}$ length $\left(f^{n_{i}}\left(\gamma^{u}\right)\right)$ with $\sigma_{0}>1$. Consequently, the sum is bounded by a multiple of length $\left(f^{n_{i}}\left(\gamma^{u}\right)\right)$. Next, one shows that the sum over a bound period $\left[n_{i}+1, n_{i}+p_{i+1}\right]$ is bounded by $C \ell_{i} / d_{i}$ :

$$
\sum_{j \in n_{i-1}+1}^{n_{i}+p_{i+1}} \operatorname{length}\left(f^{j}\left(\gamma^{2}\right)\right) \leq C \ell_{i} e^{r_{i}} .
$$

In brief terms, the ratio between length $\left(f^{j}\left(\gamma^{u}\right)\right)$ and the $\operatorname{dist}\left(f^{j}\left(\gamma^{u}\right), f^{j}\left(\bar{\zeta}_{i}\right)\right)$ is essentially preserved during the bound period. On the other hand, this distance is bounded by a geometrically decreasing sequence $C e^{-\beta j}$, by the definition of binding periods. So, the sum of the lengths over all $n_{i}<j \leq n_{i}+p_{i+1}$ is less than

$$
C \frac{\text { length }\left(f^{n_{i}}\left(\gamma^{u}\right)\right)}{d_{\mathcal{C}}\left(f^{n_{i}}\left(\gamma^{u}\right)\right)} \leq C \ell_{i} e^{r_{i}}
$$

Clearly, $\ell_{i} \leq \ell_{i} e^{r_{i}}$. Therefore, these two estimates imply that the sum over the whole time interval $\left[0, n_{k}-1\right]$ is bounded by $C \sum_{i=0}^{k} \ell_{i} e^{r_{i}}$. Now, Propositions 2.5 and 2.8 .2 imply that lengths get expanded between any consecutive free returns: $\ell_{i} \geq \sigma \ell_{i-1}$ with $\sigma_{0}>1$. It follows that, for each fixed $r$, the sequence of all the $\ell_{i} e^{r_{i}}$ with $r_{i} \equiv r$ is geometrically increasing. Therefore, the sum of $\ell_{i} e^{r_{i}}$ over the corresponding values of $i$ is bounded by a multiple of the last term:

$$
C \sum_{i=0}^{k} \ell_{i} e^{r_{i}}=C \sum_{r>0} \sum_{i: r_{i}=r} \ell_{i} e^{r_{i}} \leq C \sum_{r>0} \ell_{i(r)} e^{r}
$$

where, by definition, $i(r)$ is the largest value of $i$ for which $r_{i}=r$. By construction, $\ell_{i} e^{r_{i}}$ is less than $C r_{i}^{-2}$ for every $i$ : this is because we defined itineraries in terms of the extended family of long leaves introduced after Remark 3.3 (incidentally, this is the only place in the proof where that is used). So,

$$
C \sum_{r>0} \ell_{i(r)} e^{r} \leq \sum_{r>0} C r^{-2} \leq C
$$

This gives the lemma.

Lemma 4.8. Given any $k \geq 1$ and $i_{0}, \ldots, i_{k-1}$,

$$
\sum_{j=0}^{n_{k}-1} \operatorname{dist}\left(f^{j}(z), f^{j}\left(z^{u}\right)\right) \leq C
$$

for every $z \in R\left(i_{0}, \ldots, i_{k-1}\right)$ and every $z^{u}$ in the unstable boundary of $R\left(i_{0}, \ldots, i_{k-1}\right)$. 
Proof. Let $\sigma^{u}$ be any of the unstable sides of $R\left(i_{0}, \ldots, i_{k-1}\right)$. Suppose first that there are no close returns before time $n_{k}$, other than $\nu_{0}=-1$. Then, cf. Corollary 3.12 , every point $z \in R\left(i_{0}, \ldots, i_{k-1}\right)$ is expanding up to time $n_{k}$. So, the temporary stable leaf $\Gamma^{n_{k}}$ of order $n_{k}$ through $z$ is long and nearly vertical. If $\Gamma^{n_{k}}$ intersects $\sigma^{u}$, let $\xi$ be the intersection point. Otherwise, it must intersect some stable side $\sigma^{s}$ of $R\left(i_{0}, \ldots, i_{k-1}\right)$, let $\xi$ be the point where $\sigma^{s}$ and $\sigma^{u}$ meet. Since $\Gamma^{n_{k}}$ and $\sigma^{s}$ are exponentially contracted during at least $n_{k}$ iterates,

$$
\sum_{j=0}^{n_{k}-1} \operatorname{dist}\left(f^{j}(z), f^{j}(\xi)\right) \leq \sum_{j=0}^{n_{k}-1}(C b)^{j} \leq C .
$$
Finally, as a consequence of Lemma 4.7 , this conclusion remains true if one replaces $\xi$ by any
other point in $\sigma^{u}$.

In general, let $\nu_{0}<\nu_{1}<\cdots<\nu_{s}<n_{k}$ be the close returns prior to $n_{k}$. We allow ourselves a slight abuse of language: take $\nu_{s+1}$ to mean $n_{k}$, and $k(s+1)$ to mean $k$, wherever they occur in this proof. By Corollary $3.12, f^{\nu_{l}+\mathrm{I}}(z)$ is expanding up to time $\mu_{l}=\nu_{l+1}-\nu_{l}-1$ for any $0 \leq l \leq s$. Let $\Gamma^{\mu_{l}}$ be the corresponding temporary stable leaf through $f^{\nu_{l}+1}(z)$, and let $\lambda^{u}$ denote the unstable side of $R\left(i_{0}, \ldots, i_{k(l+1)}\right)$ that contains $\sigma^{u}$. If $\Gamma^{\mu_{1}}$ intersects $f^{\nu_{l}+1}\left(\lambda^{u}\right)$, let $\eta_{l}$ be the point of intersection. Otherwise, $\Gamma^{\mu_{l}}$ intersects some stable side $f^{\nu_{l}+1}\left(\lambda^{s}\right)$ of $f^{\nu_{l}+1}\left(R\left(i_{0}, \ldots, i_{k(l+1)}\right)\right)$, and $\eta_{l}$ is the endpoint point shared by $f^{\nu_{l}+1}\left(\lambda^{u}\right)$ and $f^{\nu_{l}+1}\left(\lambda^{s}\right)$. In both cases, cf. Lemma 3.9 ,

$$
\begin{aligned}
\operatorname{dist}\left(f^{j}(z), f^{j-\nu_{l}-1}\left(\eta_{l}\right)\right) & \leq(C b)^{j-\nu_{l}-1}\left(\operatorname{dist}\left(f^{\nu_{l}+1}(z), \eta_{l}\right)+\operatorname{length}\left(f^{j}\left(\lambda^{s}\right)\right)\right) \\
& \leq(C b)^{j-\nu_{l}-1} 2(C b)^{\left(\nu_{l}+1\right) / 2}
\end{aligned}
$$

for all $\nu_{l}+1 \leq j \leq \nu_{l+1}$. So,

$$
\sum_{j=\nu_{l}+1}^{\nu_{l+1}} \operatorname{dist}\left(f^{j}(z), f^{j-\nu_{l}-1}\left(\eta_{l}\right)\right) \leq(C b)^{\left(\nu_{l}+1\right) / 2} .
$$

Now, by the same argument as in Lemma 4.7 ,

$$
\sum_{j=\nu_{l}+1}^{\nu_{l+1}} \operatorname{length}\left(f^{j}\left(\lambda^{u}\right)\right) \leq C \sum_{q=k(l)}^{k(l+1)} \operatorname{length}\left(f^{n_{q}}\left(\lambda^{u}\right)\right) e^{r_{q}}
$$
The term corresponding to $q=k(l)$ is bounded by $C r_{k(l)}^{-2}$. For $k(l)<q \leq k(l+1)$, by
Propositions 2.5 and 2.8 .2 ,

$$
\text { length }\left(f^{n_{q}}\left(\lambda^{u}\right)\right) \geq \sigma_{1}^{\left(n_{q}-n_{q-1}\right) / 3} \text { length }\left(f^{n_{q-1}}\left(\lambda^{u}\right)\right) .
$$

Moreover, $\left.r_{q} \leq n_{q}-\nu_{l} \leq \nu_{l+1}-\nu_{l}\right)<r_{k(l+1)}$, by the definition of $\nu_{l}, \nu_{l+1}$. It follows that the sum over all $k(l)<q \leq \bar{k}(l+1)$ is bounded by

So, given any $z^{u} \in \sigma^{u}$,

$$
C \text { length }\left(f^{n_{k(l+1)}}\left(\lambda^{u}\right)\right) e^{r_{k(l+1)}} \leq C r_{k(l+1)}^{-2}
$$

$$
\sum_{j=\nu_{l}+1}^{\nu_{i+1}} \operatorname{dist}\left(f^{j}\left(z^{u}\right), f^{j-\nu_{l}-1}\left(\eta_{l}\right)\right) \leq \sum_{j=\nu_{l}+1}^{\nu_{l+1}} \operatorname{length}\left(f^{j}\left(\lambda^{u}\right)\right) \leq C r_{k(l)}^{-2}+C r_{k(l+1)}^{-2}
$$


Putting (51) and (52) together,

$$
\sum_{j=0}^{n_{k}-1} \operatorname{dist}\left(f^{j}\left(z^{u}\right), f^{j-\nu_{l}-1}\left(\eta_{l}\right)\right) \leq \sum_{l=0}^{s}(C b)^{\left(\nu_{l}+1\right) / 2}+\sum_{l=0}^{s+1} C r_{k(l)}^{-2} .
$$

It is clear that the first term on the right is bounded by some uniform constant $C>0$. To show that the same is true about the second one, we just observe that the definition of $\nu_{l}$, combined with the first part of Lemma 3.7, gives

$$
r_{k(l)}>5\left(\nu_{l}-\nu_{l-1}\right)>5 m_{k(l)}>5 r_{k(l-1)},
$$

so that $r_{k(l)}>5^{l}$ for every $0 \leq l \leq s$.

Combining Lemmas 4.7 and 4.8 , we get that

$$
\sum_{j=0}^{n_{k}-1} \operatorname{dist}\left(f^{j}(z), f^{j}(w)\right) \leq C,
$$

for any pair of points $z, w$ in any rectangle $R\left(i_{0}, \ldots, i_{k-1}\right)$. Now Proposition 4.6 follows easily: since $J$ is a bound for the derivative of $\log |\operatorname{det}| D f||$,

$$
\log \frac{\left|\operatorname{det} D f^{n-l}\left(f^{l}(z)\right)\right|}{\left|\operatorname{det} D f^{n-l}\left(f^{l}(w)\right)\right|} \leq J \sum_{j=l}^{n-1} \operatorname{dist}\left(f^{j}(z), f^{j}(w)\right) \leq J C .
$$

\subsection{Close returns are exponentially improbable}

Given $m \geq 1$ and $i_{0}, \ldots, i_{m-1}$, let $S\left(i_{0}, \ldots, i_{m-1}\right)$ be the set of points $z \in R\left(i_{0}, \ldots, i_{m-1}\right)$ for which $n_{m}$ is a close return. Leb denotes the two-dimensional Lebesgue measure (area).

Lemma 4.9. There exist $\theta=\theta(b)>0$ such that, for any $m \geq 1$ and $i_{0}, \ldots, i_{m-1}$,

$$
\frac{\operatorname{Leb}\left(S\left(i_{0}, \ldots, i_{m-1}\right)\right)}{\operatorname{Leb}\left(R\left(i_{0}, \ldots, i_{m-1}\right)\right)} \leq \min \left\{\frac{1}{\theta} e^{-\left(n_{m}-\nu_{s}\right)}, 1-\theta\right\}
$$

where $\nu_{s}$ is the last close return before $n_{m}$.

Proof. An important consequence of the definition of itineraries in Section 3.2 is that each of the stable sides $\gamma_{0}^{s}$ and $\gamma_{1}^{s}$ of $f^{n_{m}}\left(R\left(i_{0}, \ldots, i_{m-1}\right)\right)$ must be contained in $f^{n_{m}-n_{t}}\left(\Gamma_{r_{t}, l_{t}}\left(\bar{\zeta}_{t}\right)\right)$ for some $t$ with $\nu_{s} \leq n_{t} \leq n_{m-1}$. In particular, cf. Remark 3.3,

$$
\operatorname{dist}\left(\gamma_{i}^{s}, \tilde{\zeta}_{m}\right) \geq e^{-2 \beta\left(n_{m}-n_{t}\right)} \gg e^{-4\left(n_{m}-\nu_{s}\right)} .
$$

Now there are two situations to consider, corresponding to the two possibilities in Lemma 3.8.

If the two unstable sides are in tangential position to a same critical point $\eta_{0}$ (this means that $\gamma_{0}^{s}$ and $\gamma_{1}^{s}$ are both to the left or both to the right of $\eta_{0}$, see the right hand side of Figure 2) then, by (53), $n_{m}$ is not a close return for any $z \in R\left(i_{0}, \ldots, i_{m-1}\right)$. This means that $S\left(i_{0}, \ldots, i_{m-1}\right)$ is empty, and so the lemma is trivial in this case. In what remains of the proof, 
we treat the case when each unstable side of the rectangle $f^{n_{m}}\left(R\left(i_{0}, \ldots, i_{m-1}\right)\right)$ contains a critical point, see the left hand side of Figure 2.

Let $\eta_{0}=\left(x_{0}, y_{0}\right)$ be any of these critical points, and let $v=v_{n_{m}-\nu_{-}-1}$ be a $C^{1}$ vector field on this rectangle as constructed in the previous section. Then the vertical line $\left\{x=x_{0}\right\}$ crosses $f^{n_{m}}\left(R\left(i_{0}, \ldots, i_{m-1}\right)\right)$, i.e. it intersects every integral curve of $v$. We introduce coordinates $\varphi(t, y)=v^{t}\left(x_{0}, y\right)$, where $\left(v^{t}\right)_{t \in \mathbb{R}}$ denotes the flow of $v$, and we write $z(t, y)=f^{-n_{m}}(\varphi(t, y))$. Note that, by (53), this is well defined (at least) for every $|t| \leq e^{-4\left(n_{m}-\nu_{s}\right)}$. Note also that integral curves of $v$ are nearly horizontal, e.g., as a consequence of Lemmas 3.7 .2 and 4.5. It follows that, for each fixed $y$

$$
\left\{t: r_{m}(z(t, y)) \geq 5\left(n_{m}-\nu_{s}\right)\right\} \text { is contained in }\left\{t:|t| \leq C e^{-5\left(n_{m}-\nu_{s}\right)}\right\}
$$

for some sufficiently large $C$. This implies that

$$
\text { Leb }\left\{(t, y): n_{m} \text { is a close return for } z(t, y)\right\} \leq C e^{-\left(n_{m}-\nu_{s}\right)} \operatorname{Leb}\left\{(t, y):|t| \leq e^{-4\left(n_{n}-\nu_{s}\right)}\right\} \text {. }
$$

It is easy to see that $|\operatorname{det} D \varphi(0, y)|$ is uniformly bounded away from zero and infinity. Then Lemma 4.5 implies (Liouville's formula, see e.g. [14, Section I.3]), that there exists $C>0$ such that

$$
\exp \left(-C b^{-1}\right) \leq|\operatorname{det} D \varphi(t, y)| \leq \exp \left(C b^{-1}\right) \quad \text { whenever }|t| \leq e^{-4\left(n_{m}-\nu_{s}\right)} .
$$
So, the previous inequality implies that $\left\{\varphi(t, y): n_{m}\right.$ is a close return for $\left.z(t, y)\right\}$ has Lebesgue
measure bounded by

$$
\begin{aligned}
C \exp \left(2 C b^{-1}\right) e^{-\left(n_{m}-\nu_{s}\right)} & \operatorname{Leb}\left\{\varphi(t, y):|t| \leq e^{-4\left(n_{m}-\nu_{s}\right)}\right\} \\
& \leq C \exp \left(2 C b^{-1}\right) e^{-\left(n_{m}-\nu_{s}\right)} \operatorname{Leb}\left(f^{n_{m}}\left(R\left(i_{0}, \ldots, i_{m-1}\right)\right)\right) .
\end{aligned}
$$
Now, using the distortion bound in Proposition 4.6, for $k=m, n=n_{m}, l=0$, we conclude
that

$$
\operatorname{Leb}\left(S\left(i_{0}, \ldots, i_{m-1}\right)\right) \leq C \exp \left(2 C b^{-1}\right) e^{-\left(n_{m}-\nu_{s}\right)} \operatorname{Leb}\left(R\left(i_{0}, \ldots, i_{m-1}\right)\right)
$$

(possibly for a larger constant $C$ ). This gives the first estimate in the statement of the lemma, with $1 / \theta=C \exp \left(2 C b^{-1}\right)$.

The second estimate is obtained along similar lines. Firstly, the factor $C e^{-\left(n_{m}-\nu_{s}\right)}$ in (55) can be made less than $1 / 2$ by reducing $\delta$. Thus, taking complements in (55),

$$
\begin{aligned}
\operatorname{Leb}\left\{(t, y):|t| \leq e^{-4\left(n_{m}-\nu_{s}\right)} \text { and } n_{m} \text { is not a close return for } z(t, y)\right\} \\
\qquad \frac{1}{2} \operatorname{Leb}\left\{(t, y):|t| \leq e^{-4\left(n_{m}-\nu_{s}\right)}\right\} .
\end{aligned}
$$

So, arguing as before, $\left\{\varphi(t, y):|t| \leq e^{-4\left(n_{m}-\nu_{s}\right)}\right.$ and $n_{m}$ is not a close return for $\left.z(t, y)\right\}$ has Lebesgue measure larger than

$$
\frac{1}{2} \exp \left(-2 C b^{-1}\right) \operatorname{Leb}\left\{\varphi(t, y):|t| \leq e^{-4\left(n_{m}-\nu_{s}\right)}\right\} .
$$

Now, (54) also implies that $n_{m}$ is never a close return if $|t|>e^{-4\left(n_{m}-\nu_{\diamond}\right)}$. Therefore,

$\operatorname{Leb}\left\{\varphi(t, y): n_{m}\right.$ is not a close return for $\left.z(t, y)\right\}$

$$
\geq \frac{1}{2} \exp \left(-2 C b^{-1}\right) \operatorname{Leb}\left(f^{n_{m}}\left(R\left(i_{0}, \ldots, i_{m-1}\right)\right)\right)
$$


The set on the left hand side is precisely $f^{n_{m}}\left(R\left(i_{0}, \ldots, i_{m-1}\right) \backslash S\left(i_{0}, \ldots, i_{m-1}\right)\right)$. So, using Proposition 4.6,

$$
\operatorname{Leb}\left(R\left(i_{0}, \ldots, i_{m-1}\right) \backslash S\left(i_{0}, \ldots, i_{m-1}\right)\right) \geq c \exp \left(-2 C b^{-1}\right) \operatorname{Leb}\left(R\left(i_{0}, \ldots, i_{m-1}\right)\right),
$$

for some $c>0$. Equivalently,

$$
\operatorname{Leb}\left(S\left(i_{0}, \ldots, i_{m-1}\right)\right) \leq\left(1-c \exp \left(-2 C b^{-1}\right)\right) \operatorname{Leb}\left(R\left(i_{0}, \ldots, i_{m-1}\right)\right) .
$$

This gives the second bound in the statement of the lemma, with $\theta=c \exp \left(-2 C b^{-1}\right)$ (which is compatible with the expression for $\theta$ we had found before).

Given any $k \geq 1$ and $i_{0}, \ldots, i_{k-1}$, let $H\left(i_{0}, \ldots, i_{k-1}\right)$ be the subset of $z \in R\left(i_{0}, \ldots, i_{k-1}\right)$ for which no return $n_{j}$ with $j \geq k$ is a close return. According to the next proposition, this occupies a definite fraction of the rectangle $R\left(i_{0}, \ldots, i_{k-1}\right)$, in terms of Lebesgue measure Leb.

Proposition 4.10. There is $\theta_{0}=\theta_{0}(b)>0$ such that

$$
\operatorname{Leb}\left(H\left(i_{0}, \ldots, i_{k-1}\right)\right) \geq \theta_{0} \operatorname{Leb}\left(R\left(i_{0}, \ldots, i_{k-1}\right)\right)
$$

for every $i_{0}, \ldots, i_{k-1}$ and $k \geq 1$.

Proof. Let $\nu_{s}$ be the last close return with $\nu_{s} \leq n_{k-1}$. By the previous lemma, the total Lebesgue measure of the sub-rectangles $R\left(i_{0}, \ldots, i_{k-1}, i_{k}\right)$ for which $n_{k}$ is not a close return is larger than

$$
\max \left\{\theta, 1-\theta^{-1} e^{-\left(n_{k}-\nu_{s}\right)}\right\} \operatorname{Leb}\left(R\left(i_{0}, \ldots, i_{k-1}\right)\right) .
$$

In general, given $l \geq 1$ and $i_{0}, \ldots, i_{k+l-1}$ such that neither of $n_{k}, \ldots, n_{k+l-1}$ is a close return, the Lebesgue measure of all the sub-rectangles $R\left(i_{0}, \ldots, i_{k+l-1}, i_{k+l}\right)$ for which $n_{k+l}$ is also not a close return is at least

$$
\max \left\{\theta, 1-\theta^{-1} e^{-\left(n_{k+i}-\nu_{s}\right)}\right\} \operatorname{Leb}\left(R\left(i_{0}, \ldots, i_{k+l-1}\right)\right)
$$

Noting that $n_{k+l}-\nu_{s} \geq l$, we conclude that

$$
\frac{\operatorname{Leb}\left(H\left(i_{0}, \ldots, i_{k-1}\right)\right)}{\operatorname{Leb}\left(R\left(i_{0}, \ldots, i_{k-1}\right)\right)} \geq \prod_{l=0}^{\infty} \max \left\{\theta, 1-\theta^{-1} e^{-l}\right\} \geq \theta^{q} \prod_{l=q}^{\infty}\left(1-\theta^{-1} e^{-l}\right)
$$

where $q>|\log \theta|$ can be fixed arbitrarily. We choose $q$ large enough so that $e^{-q}<\theta$. Then it suffices to take $\theta_{0}$ equal to the term on the right hand side of the last inequality.

\section{Filling the holes in}

Finally, we tie the previous results together to prove Theorems A and B. First we show that Lebesgue almost every point in the region $\Delta_{0}$ has some positive iterate contained in a long stable leaf of some point of the attractor.

Let $i_{0}$ be fixed. By Proposition 4.10, the set $H\left(i_{0}\right)$ of points $z \in R\left(i_{0}\right)$ without close returns corresponds to a positive Lebesgue measure fraction of $R\left(i_{0}\right)$. By Corollary 3.12 the 
points in $H\left(i_{0}\right)$ are expanding. Clearly, the long stable leaves through these points intersect the unstable manifold $W^{u}(P) \subset \Lambda$. Now, by construction, the complement $R\left(i_{0}\right) \backslash H\left(i_{0}\right)$ can be written as a union of rectangles $R\left(i_{0}, \ldots, i_{l(1)}\right)$, with variable $l(1)$, which we call first order gaps: $n_{l(1)}$ is the first close return. Again by Proposition 4.10, a positive fraction of each first order gap is filled-in by a set $H\left(i_{0}, \ldots, i_{l(1)}\right)$ whose points $z$ have no other close returr.. So, $f^{n_{(1)}+1}(z)$ has a long stable leaf which, moreover, intersects the attractor $\Lambda$. The complement $R\left(i_{0}, \ldots, i_{l(1)}\right) \backslash H\left(i_{0}, \ldots, i_{l(1)}\right)$ is given by a union of rectangles $R\left(i_{0}, \ldots, l(1), \ldots, l(2)\right)$, the second order gaps. Now it is clear how to proceed with the argument: Proposition 4.10 tells us that a definite fraction of each $m$ th order gap $R\left(i_{0}, \ldots, i_{m}\right), m \geq 1$, is filled-in by a subset $H\left(i_{0}, \ldots, i_{l(m)}\right)$ whose points are in the $f^{n_{l(m)}}$-preimage of a long stable leaf through a point $\xi \in \Lambda$. And $R\left(i_{0}, \ldots i_{l(m)}\right) \backslash H\left(i_{0}, \ldots i_{l(m)}\right)$ is a union of rectangles, that are the gaps of order $m+1$. In this way we conclude that

$$
H=\bigcup_{m, l(1), \cdots, l(m), i_{0}, \cdots, i_{l(m)}} H\left(i_{0}, \ldots, i_{l(m)}\right)
$$

is a full Lebesgue measure subset of $\Delta_{0}$, contained in the union $\bigcup_{\xi \in \Lambda} W^{s}(\xi)$ of the stable sets of points in $\Lambda$.

Now, to complete the proof of Theorem $A$, we prove that for almost all points $w \in B(\Lambda)$ there exists $n \geq 0$ such that $z=f^{n}(w)$ is in the region $\Delta_{0}$, as claimed in Section 3 . First of all, if $0<b \ll \delta_{*} \ll 1$ then the set $H^{+}$of points whose forward orbits remain in $[-2,2]^{2}$ but do not hit $\left[-\delta_{*}, \delta_{*}\right] \times[-2,2]$ has zero Lebesgue measure. This can be proved along well-known lines. One constructs invariant stable and unstable cone fields for $f$ in $\left([-2,2] \backslash\left[-\delta_{*}, \delta_{*}\right]\right) \times[-2,2]$. It follows that the set $H$ of points whose full orbits are contained in $\left([-2,2] \backslash\left[-\delta_{*}, \delta_{*}\right]\right) \times[-2,2]$ is uniformly hyperbolic for $f$, and $H^{+} \subset W^{s}(H)$. Since the stable set $W^{s}(H)$ of $H$ must have zero Lebesgue measure, the claim follows. Then we may restrict ourselves to points having some positive iterate in $\left[-\delta_{*}, \delta_{*}\right] \times[-2,2]$, where $\delta_{*}$ is as before. For the sequel it is convenient to distinguish two cases, depending on whether the map $f$ is orientation preserving (both eigenvalues of $D f(P)$ are negative) or orientation reversing (the contracting eigenvalue is positive, the expanding one is negative).

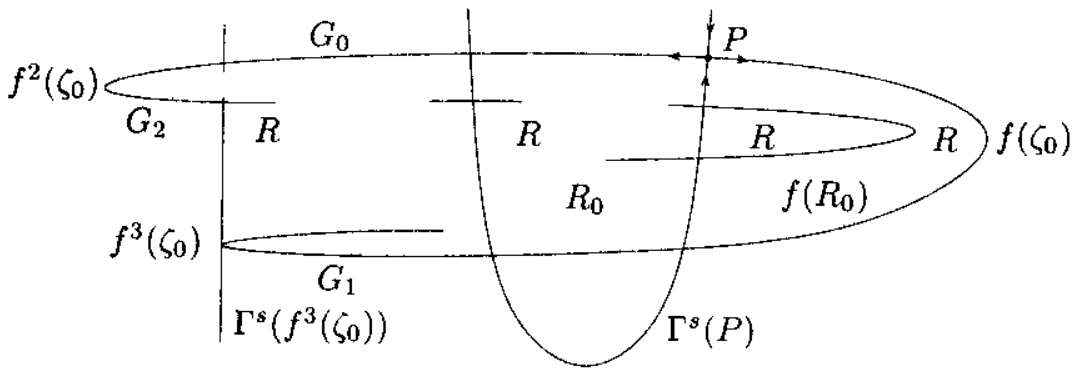

Figure 5:

The latter case is somewhat better known, see e.g. [2, Section 4], [3], [15, Section 4]. For a compact region $R$ as in Figure 5, bounded by a segment of $G_{0} \cup G_{1} \cup G_{2}$ and a segment in the long stable leaf of $f^{3}\left(\zeta_{0}\right)$, one can prove that 
1. $\Lambda$ is contained in $R$, and some fundamental domain of $f \mid W^{u}(P)$ is contained in the interior of $R$;

2. $R$ is forward invariant, i.e. $f(R) \subset R$, and is contained in the basin $B(\Lambda)$.

It follows that the union $W^{s}(P) \cup\left(\bigcup_{n \geq 0} f^{-n}(R)\right)$ contains a neighbourhood of the attractor $\Lambda$, and is contained in the basin of attraction $B(\Lambda)$. Another consequence is that the forward orbit of any point in $B(\Lambda)$ must eventually enter in $R$.

Consider the rectangle $R_{0} \subset R$ bounded by $G_{0}, G_{1}$, and the preimage under $f$ of the long stable leaf $\Gamma^{s}(P)$ passing through $P$. Recall that $G_{i}$ contains long nearly horizontal curves around $\zeta_{i}$, for $i=0,1$. Then, in view of the form of the map, each $f\left(G_{i}\right)$ must intersect $\Gamma^{s}(P)$ at exactly two points. Therefore $f^{-1}\left(\Gamma^{s}(P)\right)$ intersects $G_{i}$ at two points, and so $R_{0}$ is indeed well-defined. According to the previous paragraph, Lebesgue almost every point $w \in B(\Lambda)$ has some positive iterate $f^{k}(w)$ in $R_{0}$ (take $\delta_{*}$ small). Now, $f\left(R_{0}\right)$ is the rectangle bounded by $f\left(G_{i}\right), i=0,1$, and $\Gamma^{s}(P)$, which is clearly contained in the domain $\Delta_{0}$ defined in Section 2 . Then, by the arguments presented so far, $z=f^{k+1}(w)$ belongs Lebesgue almost surely in the stable manifold $W^{s}(\xi)$ of some point $\xi \in \Lambda$. Therefore, the same is true for $w$, and so the proof of the theorem is complete in this case.

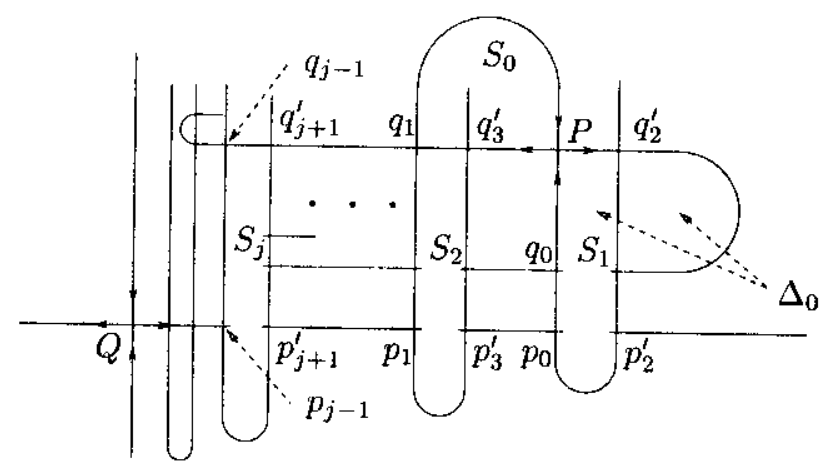

Figure 6:

Similar ideas apply when $f$ preserves orientation. It was shown in $[23$, Section 2.1] that the basin $B(\Lambda)$ contains a neighbourhood of the attractor $\Lambda$ (possibly restricting the parameter set $E$ in Theorem 2.1 to some positive Lebesgue measure subset). As part of the proof, one fixes $\delta_{*}$ with $b \ll \delta_{*} \ll 1$ in such a way that $f^{3}\left(\left[-\delta_{*}, \delta_{*}\right] \times[-2,2]\right)$ is contained in a region $S_{j}$ as in Figure 6: $S_{j}$ is bounded by a segment in $G_{0}$ and a connected piece of the stable manifold $W^{s}(P)$ of $P$ linking two nearly vertical segments of $W^{s}(P)$ through points $p_{j-1}$ and $p_{j+1}^{\prime}$ in $W^{s}(P) \cap W^{u}(Q)$. Here $Q$ is the other fixed point of $f$, located to the left of $P$. Let us denote $q_{j-1}$ and $q_{j+1}^{\prime}$, respectively, the points where these nearly vertical segments intersect $G_{0}$ (the "vertices" of $S_{j}$ ). By construction, $f\left(p_{j-1}\right)=p_{j-2}$ and $f\left(p_{j+1}^{\prime}\right)=p_{j}^{\prime}$, and then $f\left(S_{j}\right)=\tilde{S}_{j-1} \subset S_{j-1}$, where $\tilde{S}_{j-1}$ is the region bounded by $G_{1}$ and the segment of $W^{s}(P)$ connecting $f\left(q_{j-1}\right)$ to $f\left(q_{j+1}^{\prime}\right)$.

Then $f^{j+2}\left(\left[-\delta_{*}, \delta_{*}\right] \times[-2,2]\right) \subset f^{j-1}\left(S_{j}\right) \subset \tilde{S}_{1} \subset S_{1}$, note that $S_{1}$ is the region bounded by the segments of $W^{u}(P)$ and $W^{s}(P)$ linking $P$ to $q_{2}^{\prime}$. So, $f^{j+3}\left(\left[-\delta_{*}, \delta_{*}\right] \times[-2,2]\right) \subset S_{0}$, 
where $S_{0}=f\left(S_{1}\right)$ is the region bounded by the segments of $W^{u}(P)$ and $W^{s}(P)$ linking $P$ to $q_{1}=f\left(q_{2}^{\prime}\right)$. Observe, in particular, that $\zeta_{0}$ is in the boundary of $S_{0}$. What we have said so far implies that Lebesgue almost every point $w \in B(\Lambda)$ has some positive iterate $f^{k}(\omega)$ in $S_{0}$. Now, $f\left(S_{0}\right)$ is the region bounded by the segments of $W^{u}(P)$ and $W^{s}(P)$ linking $P$ to $q_{0}=f\left(q_{1}\right)$, that is, it coincides with $\Delta_{0}$. By our previous arguments, $z=f^{k+1}(w)$ belongs, Lebesgue almost surely, in the stable manifold $W^{s}(\xi)$ of some point $\xi \in \Lambda$. Then the same is true for $w$, and Theorem $\mathrm{A}$ is proved also in this case.

The proof of Theorem $A$ is complete.

Now Theorem B is a simple consequence. For each $\left(i_{0}, \ldots, i_{k}\right)$, let $\gamma$ be an unstable side of $R\left(i_{0}, \ldots, i_{k}\right)$. By Corollary 3.12 , every point in $f^{n_{k}+1}\left(H\left(i_{0}, \ldots, i_{k}\right)\right)$ has a long stable leaf, that intersects $f^{n_{k}+1}(\gamma)$ transversely. Cf. comments at the end of Section 2, almost every point in $f^{n_{k}+1}(\gamma)$ belongs to the basin $B(\mu)$. Since the set of generic points of an invariant measure consists of entire stable sets, we may conclude that $f^{n_{k}+1}\left(H\left(i_{0}, \ldots, i_{k}\right)\right) \backslash B(\mu)$ is a union of long stable leaves intersecting $f^{n_{k}+1}(\gamma)$ in a set with zero arc-length measure. The second part of Proposition 2.4 implies that the lamination of $f^{n_{k}+1}\left(H\left(i_{0}, \ldots, i_{k}\right)\right)$ by long stable leaves is Lipschitz (in the sense that the holonomy maps are Lipschitz continuous). It follows that $f^{n_{k}+1}\left(H\left(i_{0}, \ldots, i_{k}\right)\right) \backslash B(\mu)$ must have zero Lebesgue measure (area). Then the same is true for $H\left(i_{0}, \ldots, i_{k}\right) \backslash B(\mu)$. Taking the union over all $i_{0}, \ldots, i_{k}$, this proves that almost every point in set $H$ we had constructed is generic for the measure $\mu$. Since we had shown that Lebesgue almost every point in the topological basin $B(\Lambda)$ eventually reaches $H$, we conclude that $B(\Lambda) \backslash B(\mu)$ has zero area, as claimed.

This finishes the proof of Theorem $B$.

Along similar lines, we can prove that the stable manifold $W^{s}(P)$ of the fixed point $P$ is dense in the basin of attraction $B(\Lambda)$. Clearly, for this it is enough to show that $W^{s}(P)$ is dense in some full Lebesgue measure subset of $B(\Lambda)$. Thus, in view of the previous arguments in the proofs of Theorem $\mathrm{A}$ and $\mathrm{B}$, we only have to show that, given any $i_{0}, \ldots, i_{k}$, Lebesgue almost every point in $f^{n_{k}+1}\left(H\left(i_{0}, \ldots, i_{k}\right)\right)$ is in the closure of $W^{s}(P)$. Let $\gamma$ be any of the unstable sides of $R\left(i_{0}, \ldots, i_{k}\right)$. As a consequence of the result in [5, Section 3] on existence of infinitely many escape times that we commented upon at the end of Section 2, there exists a full Lebesgue measure subset $\tilde{\gamma}_{k}$ of $f^{n_{k}+1}(\gamma)$ such that any point $\xi$ in $\tilde{\gamma}_{k}$ has infinitely many returns $m_{i}=m_{i}(\xi)$ for which $f^{m_{i}}(\xi)$ is in the region between the long stable leaves $\Gamma_{\Delta}$ and $\Gamma_{\Delta+1}$. Recall Remark 3.3. So, by the Lipschitz property of the stable foliation, the subset of points whose stable leaves intersect $\tilde{\gamma}_{k}$ has full Lebesgue measure in $f^{n_{k}+1}\left(H\left(i_{0}, \ldots, i_{k}\right)\right)$. We are going to show that any point $z \in \tilde{H}_{k}$ is accumulated by $W^{s}(P)$.

We begin by noting that, since $f$ is close to a map $(x, y) \mapsto\left(1-a x^{2}, 0\right)$, with $a \approx 2$, there exists a long nearly vertical segment of $W^{s}(P)$ between $\Gamma_{\Delta}$ and $\Gamma_{\Delta+1}$. This follows from the fact that the negative orbit of the fixed point $p=(1 / 2,0)$ under the map $x \mapsto 1-2 x^{2}$ is dense in the interval $[-1,1]$, using well-known perturbation arguments. Let $\xi$ be the point where the long stable leaf through $z \in \tilde{H}_{k}$ intersects $\tilde{\gamma}_{k}$, and let $m_{i}$ be as above. Then $f^{m_{i}}(z)$ is between $\Gamma_{\Delta}$ and $\Gamma_{\Delta+1}$, and so there exists a point of $W^{s}(P)$ at distance less than $C e^{-\Delta}=C \delta$ from $f^{m_{i}}(z)$. Moreover, such a point may be chosen in the image $f^{m_{i}}\left(l_{z}\right)$ of a horizontal segment $l_{z}$ through $z$. This is because the image of $l_{z}$ is nearly horizontal near $z$, as a consequence of Propositions 2.5 and 2.8. The propositions also imply that $l_{z}$ is exponentially expanded under $f^{m_{i}}$, so $\operatorname{dist}\left(z, W^{s}(P)\right) \leq C \sigma_{0}^{-m_{i}}$ with $\sigma_{0}>1$. As $m_{i}$ may be chosen arbitrarily large, we get
that $z$ is indeed in the closure of $W^{s}(P)$. 


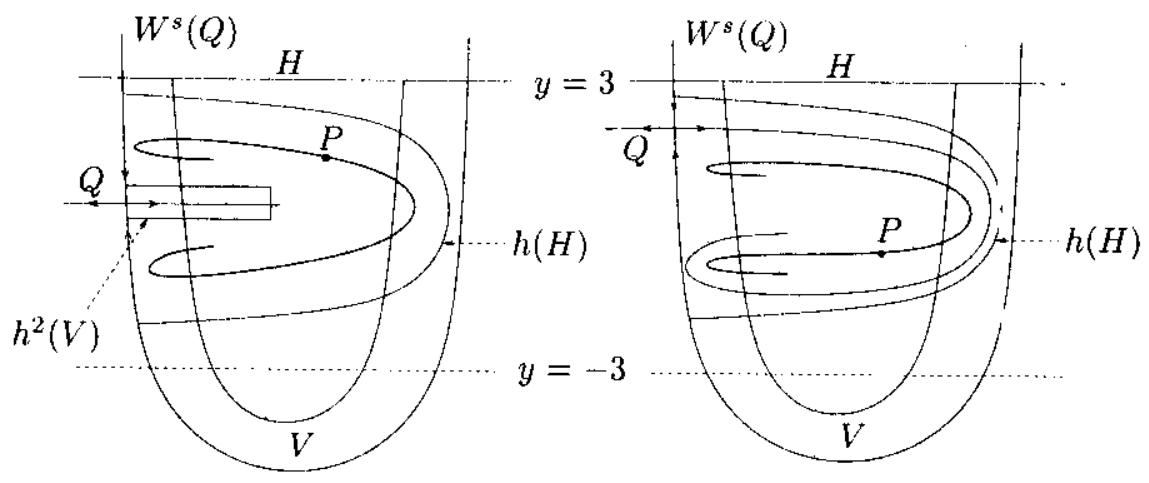

Figure 7:

Closing this paper, let us remark that for Hénon maps

$$
h(x, y)=\left(1-a x^{2}+\sqrt{b} y, \pm \sqrt{b} x\right), \quad b \text { small }
$$

it is possible to give a complete characterization of the topological basin of attraction:

(TB) the basin $B(\Lambda)$ is the domain in the plane bounded by the stable manifold $W^{s}(Q)$ of the fixed point $Q$.

This was also obtained in [9], independently of the present work. Recall that $Q$ denotes the fixed point in the region $\{x<0\}$, whereas the attractor $\Lambda$ is the closure of the other fixed point $P$. In what follows we give an outline of the proof of (TB).

First we suppose that $h$ is orientation reversing, corresponding to the positive sign in (56). See the left hand side of Figure 7 . The stable manifold of $Q$ contains two long nearly vertical segments located near $x \approx \pm 1$, that connect to each other in the region $\{y \ll 0\}$. Let $U$ be the open domain bounded by this piece of $W^{s}(Q)$ and by a horizontal line $H=\{y=3\}$. Then $U$ is forward invariant, $h(U) \subset U$, and it contains the attractor $\Lambda$.

We claim that $U$ is contained in the topological basin $B(\Lambda)$. To prove this, we begin by constructing a rectangle $V$ bordering $W^{s}(Q)$ inside $U$, such that

- $V$ contains the three first "tips" of $W^{u}(P)$, i.e., neighbourhoods of the points $f^{i}\left(\zeta_{0}\right)$, $i=1,2,3$, in Figure 5

- $h^{2}(V) \backslash V$ is contained in the domain $R$ introduced before in the context of Figure 5 .

$V$ may be constructed, e.g., using linearizing coordinates for $f$ in a neighbourhood of $Q$. We already know that $R \subset B(\Lambda)$. Since any point in $V$ must eventually leave $V$, it follows that $V \subset B(\Lambda)$. Now, let $z$ be any point in $U \backslash V$. If $z$ eventually reaches the folding region $\left[-\delta_{*}, \delta_{*}\right] \times\{|y| \leq 3\}$, then it gets mapped to $R \cup V$ in the next iterate. Otherwise, if the orbit of $z$ remains outside the folding region for all positive times, then $z$ is expanding, and so it has a long stable leaf intersecting the attractor. In both cases, $z$ is in the basin of $\Lambda$. This proves the claim. 
It follows that $B(\Lambda)$ coincides with the saturation $\bigcup_{n=0}^{\infty} h^{-n}(U)$ of the domain $U$. In particular, the boundary of $B(\Lambda)$ is contained in $W^{s}(Q) \cup \alpha(H)$, where $\alpha(H)$ is the set of accumu.ation points of the backward orbit of $H$. Now, $H$ is contained in the region $\{|y| \geq|x|$ and $y \geq 2\}$.
Using the form of the inverse map

$$
\left(x_{1}, y_{1}\right)=h^{-1}(x, y)=\left(y / \sqrt{b},\left(a y^{2} / b+x-1\right) / \sqrt{b}\right)
$$

one checks easily that

$$
|y| \geq|x| \text { and }|y| \geq 2 \quad \Rightarrow \quad\left|y_{1}\right| \geq|x| \text { and }\left|y_{1}\right| \geq 2|y| .
$$

This implies that the backward orbit of any point in that region goes off to infinity. In particular, $\alpha(H)$ is empty, and so $\partial B(\Lambda) \subset W^{s}(Q)$. On the other hand, the forward orbits of all points in a small neighbourhood of $Q$ outside $U$ also go to infinity, and so they are not in $B(\Lambda)$. Combined with the previous conclusion, this gives $\partial B(\Lambda)=W^{s}(Q)$.

Now we explain how these arguments can be adapted to the orientation preserving case $h(x, y)=\left(1-a x^{2}+\sqrt{b} y,-\sqrt{b} x\right)$. The corresponding picture is on the right hand side of Figure 7. In this case we choose the parameter $a$ in such a way that the folding region is mapped to some domain known to be contained in $B(\Lambda)$, e.g., the rectangle $S_{j}$ introduced in the context of Figure 6. We also consider a tubular neighbourhood $V$ of $W^{s}(Q)$ inside $U$, where $h$ is conjugate to a linear map. For points in $U \backslash V$, either their forward orbit eventually hits the folding region, or they have long stable leaves intersecting $\Lambda$ (because $V$ is not to thin). In either case, such points are in $B(\Lambda)$. Points in $V$ eventually move to $U \backslash V$ under forward iteration. So, the whole region $U$ is contained in the topological basin. It remains true that the backward orbit of $H=\{y=3\}$ has no (finite) accumulation points. Then the proof of (TB) proceeds precisely as in the orientation reversing case.

\section{References}

[1] V. S. Afraimovich, V. V. Bykov, and L. P. Shil'nikov. On the appearence and structure of the Lorenz attractor. Dokl. Acad. Sci. USSR, 234:336-339, 1977.

[2] M. Benedicks and L. Carleson. The dynamics of the Hénon map. Annals of Math., 133:73$169,1991$.

[3] M. Benedicks and R. Moeckel. An attractor for certain Hénon maps. Preprint ETH.

[4] M. Benedicks and M. Viana. Random perturbations and statistical properties of certain Hénon-like maps. In preparation.

[5] M. Benedicks and L.-S. Young. SBR-measures for certain Hénon maps. Invent. Math., 112:541-576, 1993.

[6] M. Benedicks and L.-S. Young. Markov extensions and decay of correlations for certain Hénon maps. Astérisque, 1999. To appear.

[7] R. Bowen. Equilibrium states and the ergodic theory of Anosov diffeomorphisms, volume 470 of Lect. Notes in Math. Springer Verlag, 1975. 
[8] R. Bowen and D. Ruelle. The ergodic theory of Axiom A flows. Invent. Math., 29:181 202, 1975.

[9] Y. Cao. The global dynamics of some Hénon maps. Preprint, 1998.

[10] L. J. Díaz, J. Rocha, and M. Viana. Strange attractors in saddle-node cycles: prevalence and globality. Invent. Math., 125:37-74, 1996.

[11] J.-P. Eckmann and D. Ruelle. Ergodic theory of chaos and strange attractors. Rev. Mod. Phys., 57:617-656, 1985 .

[12] J. Guckenheimer and R. F. Williams. Structural stability of Lorenz attractors. Publ. Math. IHES, 50:59-72, 1979.

[13] M. Jakobson and S. Newhouse. Asymptotic measures for dissipative diffeomorphisms. In preparation.

[14] R. Mañé. Ergodic theory and differentiable dynamics. Springer Verlag, 1987.

[15] L. Mora and M. Viana. Abundance of strange attractors. Acta Math., 171:1-71, 1993.

[16] J. Palis and F. Takens. Hyperbolicity and sensitive-chaotic dynamics at homoclinic bifurcations. Cambridge University Press, 1993.

[17] Ya. Pesin. Families of invariant manifolds corresponding to non-zero characteristic exponents. Math. USSR. Izv., 10:1261--1302, 1976.

[18] C. Pugh and M. Shub. Ergodic attractors. Trans. Amer. Math. Soc., 312:1 54, 1989.

[19] D. Ruelle. A measure associated with Axiom A attractors. Amer. J. Math., 98:619-654, 1976.

[20] Ya. Sinai. Gibbs measure in ergodic theory. Russian Math. Surveys, 27:21-69, 1972.

[21] S. Smale. Differentiable dynamical systems. Bull. Am. Math. Soc., 73:747-817, 1967.

[22] M. Viana. Strange attractors in higher dimensions. Bull. Braz. Math. Soc., 24:13-62, 1993.

[23] M. Viana. Global attractors and bifurcations. In Nonlinear Dynamical Systems and Chaos, volume 19 of Progress in NLPDE and Applications, pages 317--344. Birkhäuser, 1996.

Michael Benedicks: KTH, Stockholm, michaelb@math.kth.se

Marcelo Viana: IMPA, Rio de Janeiro, viana@impa.br 